\title{
FRAGMENTATION ASSOCIATED TO LÉVY PROCESSES USING SNAKE
}

\author{
ROMAIN ABRAHAM AND JEAN-FRANÇOIS DELMAS
}

\begin{abstract}
We consider the height process of a Lévy process with no negative jumps, and its associated continuous tree representation. Using Lévy snake tools developed by Duquesne and Le Gall, with an underlying Poisson process, we construct a fragmentation process, which in the stable case corresponds to the self-similar fragmentation described by Miermont. For the general fragmentation process we compute a family of dislocation measures as well as the law of the size of a tagged fragment. We also give a special Markov property for the snake which is interesting in itself.
\end{abstract}

\section{INTRODUCTION}

We present a fragmentation process associated to general critical or sub-critical continuous random trees (CRT) which were introduced by Le Gall and Le Jan [15] and developed later by Duquesne and Le Gall [10]. This extends previous work from Miermont [18] on stable CRT. Although the underlying ideas are the same in both constructions, the arguments in the proofs are very different. Following Abraham and Serlet [1 who deal with the particular case of Brownian CRT, our arguments rely on Lévy Poisson Snake processes. Those path processes are Lévy Snake, see [10], with underlying Poisson process. To prove the fragmentation property, we need some results on Lévy Snake which are interesting by themselves. Eventually we give the dislocation measure of the fragmentation process. We think this construction provides non trivial examples of non self-similar fragmentations, and that the tools developed here could give further results on the fragmentation associated to CRT.

The next three subsections give a brief presentation of the mathematical objects and state the mains results. The last one describes the organization of the paper.

1.1. Exploration process. The coding of a tree by its height process is now well-known. For instance, the height process of Aldous' CRT [2 is a normalized Brownian excursion. In 15], Le Gall and Le Jan associated to a Lévy process with no negative jumps that does not drift to infinity, $X=\left(X_{t}, t \geq 0\right)$, a continuous state branching process (CSBP) and a Lévy CRT which keeps track of the genealogy of the CSBP. Let $\psi$ denote the Laplace exponent of $X$. We shall assume there is no Brownian part, so that

$$
\psi(\lambda)=\alpha_{0} \lambda+\int_{(0,+\infty)} \pi(d \ell)\left[\mathrm{e}^{-\lambda \ell}-1+\lambda \ell\right]
$$

with $\alpha_{0} \geq 0$ and the Lévy measure $\pi$ is a positive $\sigma$-finite measure on $(0,+\infty)$ such that $\int_{(0,+\infty)}\left(\ell \wedge \ell^{2}\right) \pi(d \ell)<\infty$. Following [10], we shall also assume that $X$ is of infinite variation

Date: September 5, 2018.

2000 Mathematics Subject Classification. 60J25, 60G57.

Key words and phrases. Fragmentation, Lévy snake, dislocation measure, stable processes, special Markov property. 
a.s. which implies that $\int_{(0,1)} \ell \pi(d \ell)=\infty$. Notice those hypothesis are fulfilled in the stable case: $\psi(\lambda)=\lambda^{\alpha}, \alpha \in(1,2)$.

Informally for the height process, $H=\left(H_{t}, t \geq 0\right), H_{t}$ gives the distance (which can be understood as the number of generations) between the individual labeled $t$ and the root, 0 , of the CRT. This process is a key tool in this construction but it is not a Markov process. The so-called exploration process $\rho=\left(\rho_{t}, t \geq 0\right)$ is a càd-làg Markov process taking values in $\mathcal{M}_{f}\left(\mathbb{R}_{+}\right)$, the set of finite measure on $\mathbb{R}_{+}$, endowed with the topology of weak convergence. The height process can easily be recovered from the exploration process as $H_{t}=H\left(\rho_{t}\right)$, where $H(\mu)$ denotes the supremum of the closed support of the measure $\mu$ (with the convention that $\mathrm{H}(0)=0)$. In some sense $\rho_{t}(d v)$ records the "number" of brothers, with labels larger than $t$, of the ancestor of $t$ at generation $v$. Furthermore the jumps of $\rho$ are given by

$$
\rho_{t}-\rho_{t-}=\Delta_{t} \delta_{H_{t}},
$$

where $\Delta_{t}$ is the jump of the Lévy process $X$ at time $t$ and $\delta_{x}$ is the Dirac mass at $x$. Intuitively $\Delta_{t}$ represents the "size" of the progeny of such individual $t$. And the set $\{s \geq$ $\left.t ; \min \left\{H_{u}, u \in[t, s]\right\} \geq H_{t}\right\}$ represents the "size" of the total descendants of the individual $t$. Such individual $t$ corresponds to a node in the CRT. To each jump of $X$ corresponds a node in the CRT and vice-versa. Definition and properties of the height process and exploration process are recalled in Section 2 .

1.2. Fragmentation. A fragmentation process is a Markov process which describes how an object with given total mass evolves as it breaks into several fragments randomly as time passes. Notice there may be loss of mass but no creation. This kind of processes has been widely studied in the recent years, see Bertoin [7] and references therein. To be more precise, the state space of a fragmentation process is the set of the non-increasing sequences of masses with finite total mass

$$
\mathcal{S}^{\downarrow}=\left\{s=\left(s_{1}, s_{2}, \ldots\right) ; s_{1} \geq s_{2} \geq \cdots \geq 0 \quad \text { and } \quad \Sigma(s)=\sum_{k=1}^{+\infty} s_{k}<+\infty\right\} .
$$

If we denote by $P_{s}$ the law of a $\mathcal{S}^{\downarrow}$-valued process $\Lambda=\left(\Lambda^{\theta}, \theta \geq 0\right)$ starting at $s=\left(s_{1}, s_{2}, \ldots\right) \in$ $\mathcal{S}^{\downarrow}$, we say that $\Lambda$ is a fragmentation process if it is a Markov process such that $\theta \mapsto \Sigma\left(\Lambda^{\theta}\right)$ is non-increasing and if it fulfills the fragmentation property: the law of $\left(\Lambda^{\theta}, \theta \geq 0\right)$ under $P_{s}$ is the non-increasing reordering of the fragments of independent processes of respective laws $P_{\left(s_{1}, 0, \ldots\right)}, P_{\left(s_{2}, 0, \ldots\right)}, \ldots$ In other words, each fragment after dislocation behaves independently of the others, and its evolution depends only on its initial mass. As a consequence, to describe the law of the fragmentation process with any initial condition, it suffices to study the laws $P_{r}:=P_{(r, 0, \ldots)}$ for any $r \in(0,+\infty)$, i.e. the law of the fragmentation process starting with a single mass $r$.

A fragmentation process is said to be self-similar of index $\alpha$ if, for any $r>0$, the law of the process $\left(\Lambda^{\theta}, \theta \geq 0\right)$ under $P_{r}$ is the law of the process $\left(r \Lambda^{r^{\alpha} \theta}, \theta \geq 0\right)$ under $P_{1}$. Bertoin 6 ] proved that the law of a self-similar fragmentation is characterized by: the index of selfsimilarity $\alpha$, an erosion coefficient which corresponds to a deterministic rate of mass loss, and a dislocation measure $\nu$ on $\mathcal{S}^{\downarrow}$ which describes sudden dislocations of a fragment of mass 1 .

Connections between fragmentation processes and random trees or Brownian excursion have been pointed out by several authors. Let us mention the work of Bertoin [5] who constructed a fragmentation process by looking at the lengths of the excursions above level $t$ of a Brownian excursion. Aldous and Pitman [3] constructed another fragmentation process, which is related to the additive coalescent process, by cutting Aldous' Brownian CRT. Their 
proofs rely on projective limits on trees. Those results have been generalized, by Miermont [17, 18 to CRT associated to stable Lévy processes, using path transformations of the Lévy process. Concerning the Aldous-Pitman's fragmentation process, Abraham and Serlet 1 give an alternative construction using Poisson snakes. Our presentation follow their ideas. However, we give next a more intuitive presentation which is in fact equivalent (see Section 9.1).

We consider an excursion of the Lévy process $X$ out of 0 , which correspond also to an excursion of the exploration process (and the height process) out of 0 . Let $\sigma$ denote the common length of those excursions. Intuitively, $\sigma$ represents the "size" of the total progeny of the root 0 . Let $\mathcal{J}=\left\{t \in[0, \sigma] ; X_{t} \neq X_{t-}\right\}$ the set of jumping times of $X$ or nodes of the CRT, and consider $\left(T_{t} ; t \in \mathcal{J}\right)$ a countable family of independent random variable such that $T_{t}$ is distributed according to an exponential law with parameter $\Delta_{t}$. At time $T_{t}$, the node corresponding to the jump $\Delta_{t}$ is cut from the CRT. Two individuals, say $u \leq v$, belongs to the same fragment at time $\theta$ if no node has been cut before time $\theta$ between them and their most recent common ancestor which is defined as $u \curlywedge v=\inf \left\{t \in[0, u] ; \min \left\{H_{r}, r \in[u, v]\right\}=\right.$ $\left.\min \left\{H_{r}, r \in[t, u]\right\}\right\}$. Let $\Lambda^{\theta}$ denote the family of decreasing positive Lebesgue measure of the fragments completed by zeros if necessary so that $\Lambda^{\theta} \in \mathcal{S}^{\downarrow}$. See Section 9.1 for a precise construction.

Cutting nodes at time $\theta>0$ may be viewed as adding horizontal lines under the epigraph of $H$ (see figure 1).

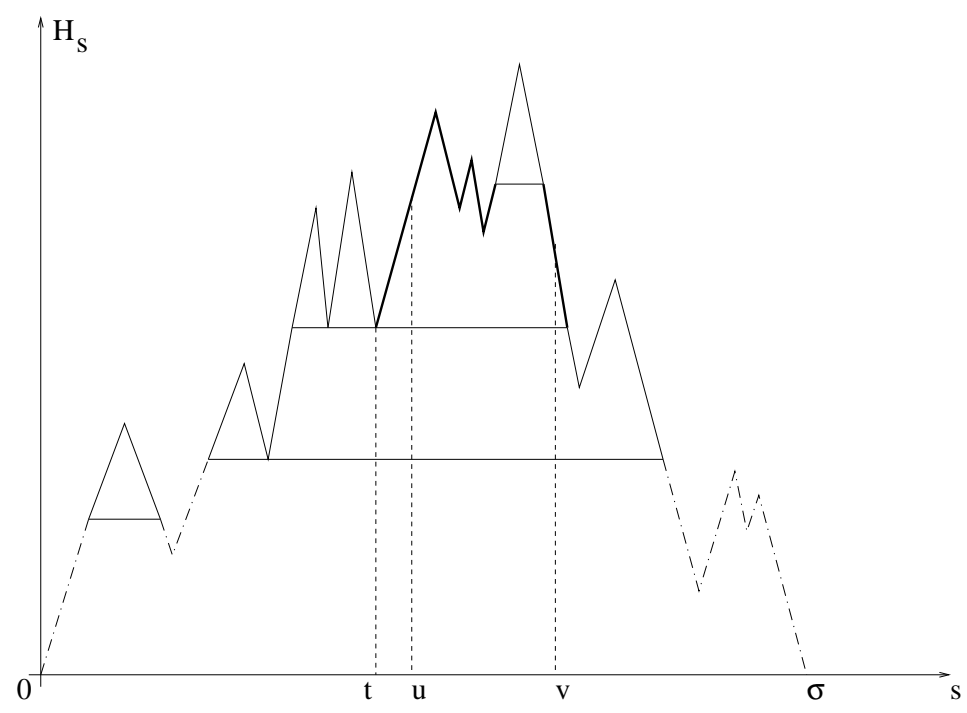

Figure 1. Cutting at nodes: a modifier

We then consider the excursions obtained after cutting the initial excursion along the horizontal lines and gluing together the corresponding pieces of paths (for instance, the bold piece of the path of $H$ in Figure 1 corresponds to the bold excursion in Figure 2). The lengths of these excursions, ranked in decreasing order, form the fragmentation process as $\theta$ increases. Of course, the figure are caricatures as the process $H$ is very irregular and the number of fragments is infinite.

Theorem 8.3 asserts that the process $\left(\Lambda^{\theta}, \theta \geq 0\right)$ is a fragmentation process. There is no loss of mass thanks to Proposition 8.8, 


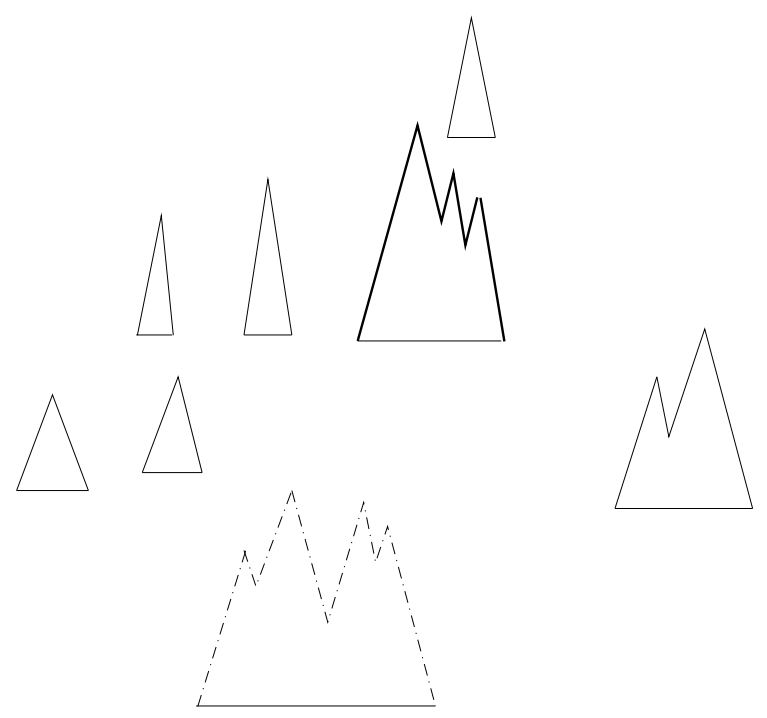

FiguRE 2. Fragmentation of the excursion

In the stable case, $\psi(\lambda)=\lambda^{\alpha}$ with $\alpha \in(1,2)$, using scaling properties, we get the fragmentation is self-similar with index $1 / \alpha$ and we recover the results of Miermont [18, see Corollary 9.3. In particular the dislocation measure is given by: for any measurable non-negative function $F$ on $\mathcal{S}^{\downarrow}$,

$$
\int F(x) \nu(d x)=\frac{\alpha(\alpha-1) \Gamma([\alpha-1] / \alpha)}{\Gamma(2-\alpha)} \mathbb{E}\left[S_{1} F\left(\Delta S_{t} / S_{1}, t \leq 1\right)\right],
$$

where $\left(S_{t}, t \geq 0\right)$ is a stable subordinator with Laplace exponent $\psi^{-1}(\lambda)=\lambda^{1 / \alpha}$, and $F\left(\Delta S_{t} / S_{1}, t \leq 1\right)$ has to be understood as $F$ applied to the decreasing reordering of the sequence $\left(\Delta S_{t} / S_{1}, t \leq 1\right)$.

In the general case, the fragmentation is not self-similar. However, if $\mathcal{T}=\left\{\theta \geq 0 ; \Lambda^{\theta} \neq\right.$ $\left.\Lambda^{\theta-}\right\}$ denotes the jumping times of the process $\Lambda$, we get as a direct consequence of Section 9.3 that

$$
\sum_{\theta \in \mathcal{T}} \delta_{\theta, \Lambda^{\theta}}
$$

is a point process with intensity $d \theta \tilde{\nu}_{\Lambda^{\theta-}}(d s)$, where $\left(\tilde{\nu}_{x}, x \in \mathcal{S}^{\downarrow}\right)$ is a family of $\sigma$-finite measures on $\mathcal{S}^{\downarrow}$. There exists a family $\left(\nu_{r}, r>0\right)$ of $\sigma$-finite measure on $\mathcal{S}^{\downarrow}$, which we call dislocation measures of the fragmentation $\Lambda$, such that for any $x=\left(x_{1}, x_{2}, \ldots\right) \in \mathcal{S}^{\downarrow}$ and any nonnegative measurable function, $F$, defined on $\mathcal{S}^{\downarrow}$,

$$
\int F(s) \tilde{\nu}_{x}(d s)=\sum_{i \in \mathbb{N}^{*} ; x_{i}>0} \int F\left(x^{i, s}\right) \nu_{x_{i}}(d s),
$$

where $x^{i, s}$ is the decreasing reordering of the merging of the sequences $s \in \mathcal{S}^{\downarrow}$ and $x$, where $x_{i}$ has been removed of the sequence $x$. This means that only one element of $x$ fragments and the fragmentation depends only on the size of this very fragment. The dislocation measures can be computed, see Theorem 9.1 In particular $\nu_{r}(d x)$-a.e. $\sum_{i \in \mathbb{N}^{*}} x_{i}=r$ assures there is no loss of mass at the dislocation. The definition of the dislocation measures is more involved 
than in the stable case. However, it can still be written using the law of the jumps of a subordinator with Laplace exponent $\psi^{-1}$.

1.3. The pruned exploration process. In fact the dislocation measure is computed by studying the evolution of a tagged fragment, for example the one that contains the root of the CRT. Therefore, it is natural to consider first the exploration process of the fragment containing the root at time $\theta$. The pruned exploration process, $\tilde{\rho}=\left(\tilde{\rho}_{t}, t \geq 0\right)$, is defined by $\tilde{\rho}_{t}=\rho_{C_{t}}$, where $C_{t}=\inf \left\{r>0 ; A_{r} \geq t\right\}$ is the right continuous inverse of $A_{t}$, the Lebesgue measure of the set of individuals prior to $t$ who belongs to the tagged fragment at time $\theta$ (Section (4). The pruned process $\tilde{\rho}$ corresponds to the exploration process associated to the dashed height process of Figures 1 and 2]. To get the law of the pruned exploration process (Section [6), we use a Poisson Lévy snake approach (Section [3) and we prove a special Markov property, Theorem [5.2] in Section [5] which is of independent interest. Notice this theorem differs from Proposition 4.2.3 in [10], or Proposition 7 in [8], where in both cases the exit measure is singular, whereas here it is absolutely continuous w.r.t. to the Lebesgue measure.

Eventually, using martingales, we get Theorem 6.1 the pruned exploration process $\tilde{\rho}$ is the exploration process associated to a Lévy process, $X^{(\theta)}$, with Laplace exponent $\psi^{(\theta)}$ defined by: for $\lambda \in \mathbb{R}_{+}$,

$$
\psi^{(\theta)}(\lambda)=\psi(\lambda+\theta)-\psi(\theta) .
$$

There exists other pruning procedure for Galton-Watson trees, see for example [1] and references therein.

Notice that conditionally on the length of the excursion, the excursions of $X$ and $X^{(\theta)}$ out of 0 are equally distributed (see Lemma 7.11). This property, as well as the special Markov property are essential to prove the fragmentation property. We also compute, see Proposition 7.3 the joint law of $\sigma$, the initial mass of the fragment, and $\tilde{\sigma}$ the mass of the tagged fragment at time $\theta$, under the excursion measure.

1.4. Organization of the paper. In Section 2 we recall the construction of the Lévy CRT and give the properties we shall use in this paper. Section 3 is devoted to the definition and some properties of the Lévy Poisson snake. From this Lévy Poisson snake, we define in Section 4 the pruned exploration process which corresponds to the tagged fragment that contains 0. Then, we introduce in Section [5 a special Markov property for the Lévy Poisson snake: Theorem 5.2 and Corollary 5.3. We compute in Section [6 the law of the pruned exploration process, see Theorem [6.1. Section 7 is then devoted to the study of some properties of the pruned exploration process under the excursion measure. Eventually, we construct in Section 8 the fragmentation process associated to our Lévy Poisson snake and prove the fragmentation property, Theorem 8.3 , and check there is no loss of mass, Proposition 8.8 , In Section 9, we identify completely the law of the fragmentation process by computing the dislocation measures, Theorem 9.1, and we recover the result of Miermont 18, for the stable case in Corollary 9.3 .

\section{LÉVY SNAKE: NOTATIONS AND PROPERTIES}

We recall here the construction of the Lévy continuous random tree (CRT) introduced in [15, 14] and developed later in [10. We will emphasize on the height process and the exploration process which are the key tools to handle this tree. The results of this section are mainly extract from 10 . 
2.1. The underlying Lévy process. We consider a $\mathbb{R}$-valued Lévy process $\left(X_{t}, t \geq 0\right)$ with no negative jumps, starting from 0 . Its law is characterized by its Laplace transform: for $\lambda \geq 0$

$$
\mathbb{E}\left[\mathrm{e}^{-\lambda X_{t}}\right]=\mathrm{e}^{t \psi(\lambda)}
$$

where its Laplace exponent, $\psi$, is given by

$$
\psi(\lambda)=\alpha_{0} \lambda+\int_{(0,+\infty)} \pi(d \ell)\left[\mathrm{e}^{-\lambda \ell}-1+\lambda \ell\right]
$$

with $\alpha_{0} \geq 0$ and the Lévy measure $\pi$ is a positive $\sigma$-finite measure on $(0,+\infty)$ such that

$$
\int_{(0,+\infty)}\left(\ell \wedge \ell^{2}\right) \pi(d \ell)<\infty \text { and } \int_{(0,1)} \ell \pi(d \ell)=\infty .
$$

The first assumption (with the condition $\alpha_{0} \geq 0$ ) implies the process $X$ does not drift to infinity, while the second implies $X$ is of infinite variation a.s.

For $\lambda \geq 1 / \varepsilon>0$, we have $\mathrm{e}^{-\lambda \ell}-1+\lambda \ell \geq \frac{1}{2} \lambda \ell \mathbf{1}_{\{\ell \geq 2 \varepsilon\}}$, which implies that $\lambda^{-1} \psi(\lambda) \geq$ $\alpha_{0}+\int_{(2 \varepsilon, \infty)} \ell \pi(d \ell)$. We deduce that

$$
\lim _{\lambda \rightarrow \infty} \frac{\lambda}{\psi(\lambda)}=0
$$

We introduce some processes related to $X$. Let $\mathcal{J}=\left\{s \geq 0 ; X_{s} \neq X_{s-}\right\}$, the set of jumping times of $X$. For $s \in \mathcal{J}$, we denote by

$$
\Delta_{s}=X_{s}-X_{s-}
$$

the jump of $X$ at time $s$ and $\Delta_{s}=0$ otherwise. The random measure $\mathcal{X}=\sum_{s \in \mathcal{J}} \delta_{s, \Delta_{s}}$ is a Poisson point process with intensity $\pi(d \ell)$. Let $I=\left(I_{t}, t \geq 0\right)$ be the infimum process of $X$, $I_{t}=\inf _{0 \leq s \leq t} X_{s}$, and let $S=\left(S_{t}, t \geq 0\right)$ be the supremum process, $S_{t}=\sup _{0 \leq s \leq t} X_{s}$. We will also consider for every $0 \leq s \leq t$ the infimum of $X$ over $[s, t]$ :

$$
I_{t}^{s}=\inf _{s \leq r \leq t} X_{r} .
$$

The point 0 is regular for the Markov process $X-I$, and $-I$ is the local time of $X-I$ at 0 (see [4], chap. VII). Let $\mathbb{N}$ be the associated excursion measure of the process $X-I$ out of 0 , and $\sigma=\inf \left\{t>0 ; X_{t}-I_{t}=0\right\}$ the length of the excursion of $X-I$ under $\mathbb{N}$. We will assume that under $\mathbb{N}, X_{0}=I_{0}=0$.

Since $X$ is of infinite variation, 0 is also regular for the Markov process $S-X$. The local time, $L=\left(L_{t}, t \geq 0\right)$, of $S-X$ at 0 will be normalized so that

$$
\mathbb{E}\left[\mathrm{e}^{-\beta S_{L_{t}^{-1}}}\right]=\mathrm{e}^{-t \psi(\beta) / \beta},
$$

where $L_{t}^{-1}=\inf \left\{s \geq 0 ; L_{s} \geq t\right\}$ (see also [4] Theorem VII.4 (ii)).

2.2. The height process and the Lévy CRT. For each $t \geq 0$, we consider the reversed process at time $t, \hat{X}^{(t)}=\left(\hat{X}_{s}^{(t)}, 0 \leq s \leq t\right)$ by:

$$
\hat{X}_{s}^{(t)}=X_{t}-X_{(t-s)-} \quad \text { if } \quad 0 \leq s<t,
$$

and $\hat{X}_{t}^{(t)}=X_{t}$. The two processes $\left(\hat{X}_{s}^{(t)}, 0 \leq s \leq t\right)$ and $\left(X_{s}, 0 \leq s \leq t\right)$ have the same law. Let $\hat{S}^{(t)}$ be the supremum process of $\hat{X}^{(t)}$ and $\hat{L}^{(t)}$ be the local time at 0 of $\hat{S}^{(t)}-\hat{X}^{(t)}$ with the same normalization as $L$. 
Definition 2.1. There exists a process $H=\left(H_{t}, t \geq 0\right)$, called the height process, such that for all $t \geq 0$, a.s. $H_{t}=\hat{L}_{t}^{(t)}$, and $H_{0}=0$. Furthermore $H$ is lower semi-continuous a.s. and a.s. for all $t^{\prime}>t \geq 0$, the process $H$ takes all the values between $H_{t}$ and $H_{t^{\prime}}$ on the time interval $\left[t, t^{\prime}\right]$.

The height process $\left(H_{t}, t \in[0, \sigma]\right)$ under $\mathbb{N}$ codes a continuous genealogical structure, the Lévy CRT, via the following procedure.

(i) To each $t \in[0, \sigma]$ corresponds a vertex at generation $H_{t}$.

(ii) Vertex $t$ is an ancestor of vertex $t^{\prime}$ if $H_{t}=H_{t, t^{\prime}}$, where

$$
H_{t, t^{\prime}}=\inf \left\{H_{u}, u \in\left[t \wedge t^{\prime}, t \vee t^{\prime}\right]\right\} .
$$

In general $H_{t, t^{\prime}}$ is the generation of the last common ancestor to $t$ and $t^{\prime}$.

(iii) We put $d\left(t, t^{\prime}\right)=H_{t}+H_{t^{\prime}}-2 H_{t, t^{\prime}}$ and identify $t$ and $t^{\prime}\left(t \sim t^{\prime}\right)$ if $d\left(t, t^{\prime}\right)=0$.

The Lévy CRT coded by $H$ is then the quotient set $[0, \sigma] / \sim$, equipped with the distance $d$ and the genealogical relation specified in (ii).

2.3. The exploration process. The height process is in general not Markov. But it is a very simple function of a measure-valued Markov process, the so-called exploration process.

If $E$ is a polish space, let $\mathcal{B}(E)$ (resp. $\mathcal{B}_{+}(E)$ ) be the set of real-valued measurable (resp. and non-negative) functions defined on $E$ endowed with its Borel $\sigma$-field, and let $\mathcal{M}(E)$ (resp. $\left.\mathcal{M}_{f}(E)\right)$ be the set of $\sigma$-finite (resp. finite) measures on $E$, endowed with the topology of vague (resp. weak) convergence. For any measure $\mu \in \mathcal{M}(E)$ and $f \in \mathcal{B}_{+}(E)$, we write

$$
\langle\mu, f\rangle=\int f(x) \mu(d x) .
$$

The exploration process $\rho=\left(\rho_{t}, t \geq 0\right)$ is a $\mathcal{M}_{f}\left(\mathbb{R}_{+}\right)$-valued process defined as follows: for every $f \in \mathcal{B}_{+}\left(\mathbb{R}_{+}\right)$,

$$
\left\langle\rho_{t}, f\right\rangle=\int_{[0, t]} d_{s} I_{t}^{s} f\left(H_{s}\right)
$$

or equivalently

$$
\rho_{t}(d r)=\sum_{\substack{0<s \leq t \\ X_{s-}<I_{t}^{s}}}\left(I_{t}^{s}-X_{s-}\right) \delta_{H_{s}}(d r) .
$$

In particular, the total mass of $\rho_{t}$ is $\left\langle\rho_{t}, 1\right\rangle=X_{t}-I_{t}$.

For $\mu \in \mathcal{M}\left(\mathbb{R}_{+}\right)$, we set

$$
H(\mu)=\sup \operatorname{Supp} \mu,
$$

where Supp $\mu$ is the closed support of $\mu$, with the convention $H(0)=0$. We have

Proposition 2.2. Almost surely, for every $t>0$,

- $H\left(\rho_{t}\right)=H_{t}$,

- $\rho_{t}=0$ if and only if $H_{t}=0$,

- if $\rho_{t} \neq 0$, then Supp $\rho_{t}=\left[0, H_{t}\right]$.

- $\rho_{t}=\rho_{t^{-}}+\Delta_{t} \delta_{H_{t}}$, where $\Delta_{t}=0$ if $t \notin \mathcal{J}$.

In the definition of the exploration process, as $X$ starts from 0 , we have $\rho_{0}=0$ a.s. To get the Markov property of $\rho$, we must define the process $\rho$ started at any initial measure $\mu \in \mathcal{M}_{f}\left(\mathbb{R}_{+}\right)$. 
For $a \in[0,\langle\mu, 1\rangle]$, we define the erased measure $k_{a} \mu$ by

$$
k_{a} \mu([0, r])=\mu([0, r]) \wedge(\langle\mu, 1\rangle-a), \quad \text { for } r \geq 0 .
$$

If $a>\langle\mu, 1\rangle$, we set $k_{a} \mu=0$. In other words, the measure $k_{a} \mu$ is the measure $\mu$ erased by a mass $a$ backward from $H(\mu)$.

For $\nu, \mu \in \mathcal{M}_{f}\left(\mathbb{R}_{+}\right)$, and $\mu$ with compact support, we define the concatenation $[\mu, \nu] \in$ $\mathcal{M}_{f}\left(\mathbb{R}_{+}\right)$of the two measures by:

$$
\langle[\mu, \nu], f\rangle=\langle\mu, f\rangle+\langle\nu, f(H(\mu)+\cdot)\rangle, \quad f \in \mathcal{B}_{+}\left(\mathbb{R}_{+}\right) .
$$

Eventually, we set for every $\mu \in \mathcal{M}_{f}\left(\mathbb{R}_{+}\right)$and every $t>0 \rho_{t}^{\mu}=\left[k_{-I_{t}} \mu, \rho_{t}\right]$. We say that $\left(\rho_{t}^{\mu}, t \geq 0\right)$ is the process $\rho$ started at $\rho_{0}^{\mu}=\mu$, and write $\mathbb{P}_{\mu}$ for its law. Unless there is an ambiguity, we shall write $\rho_{t}$ for $\rho_{t}^{\mu}$.

Proposition 2.3. The process $\left(\rho_{t}, t \geq 0\right)$ is a càd-làg strong Markov process in $\mathcal{M}_{f}\left(\mathbb{R}_{+}\right)$.

Remark 2.4. From the construction of $\rho$, we get that a.s. $\rho_{t}=0$ if and only if $-I_{t} \geq\left\langle\rho_{0}, 1\right\rangle$ and $X_{t}-I_{t}=0$. This implies that 0 is also a regular point for $\rho$. Let $\left(\tau_{s}, s \geq 0\right)$ be the right continuous inverse of $-I: \tau_{s}=\inf \left\{t>0 ;-I_{t}>s\right\}$. We get the local time at 0 of $\rho^{\mu}$, $\left(L_{t}^{0}, t \geq 0\right)$, is given for $t \geq 0$, by

$$
L_{t}^{0}=-I_{t}+I_{t \wedge \tau_{\langle\mu, 1\rangle}} .
$$

Notice that $\mathbb{N}$ is also the excursion measure of the process $\rho$ out of 0 , and that $\sigma$, the length of the excursion, is $\mathbb{N}$-a.e. equal to $\inf \left\{t>0 ; \rho_{t}=0\right\}$.

Remark 2.5. Recall $\left(\Delta_{s}, s \geq 0\right)$ are the jumps of the process $X$. The process $\rho$ is adapted to the filtration generated by the process $X$, that is by the Poisson point process $\mathcal{X}$, and $\rho_{0}$, completed the usual way. From the construction of $\rho$, we get there exists a measurable function, $\Gamma$, defined on $\mathcal{M}\left(\mathbb{R}_{+}^{2}\right) \times \mathcal{M}_{f}\left(\mathbb{R}_{+}\right)$(endowed with its Borel $\sigma$-field) taking values in $\mathcal{M}_{f}\left(\mathbb{R}_{+}\right)$(endowed with its Borel $\sigma$-field), such that

$$
\rho_{t}=\Gamma\left(\mathcal{X} \mathbf{1}_{[0, t] \times \mathbb{R}_{+}}, \rho_{0}\right) .
$$

On the other hand, notice that a.s. the jumping times of $\rho$ are also the jumping times of $X$, and for $s \in \mathcal{J}$, we have $\rho_{s}\left(\left\{H_{s}\right\}\right)=\Delta_{s}$. We deduce that $\left(\Delta_{u}, u \in(s, t]\right)$ is measurable w.r.t. the $\sigma$-field $\sigma\left(\rho_{u}, u \in[s, t]\right)$.

2.4. The dual process and representation formula. We shall need the $\mathcal{M}_{f}\left(\mathbb{R}_{+}\right)$-valued process $\eta=\left(\eta_{t}, t \geq 0\right)$ defined by

$$
\eta_{t}(d r)=\sum_{\substack{0<s \leq t \\ X_{s}<I_{t}^{s}}}\left(X_{s}-I_{t}^{s}\right) \delta_{H_{s}}(d r) .
$$

The process $\eta$ is the dual process of $\rho$ under $\mathbb{N}$ (see Corollary 3.1.6 in [10]). We write (recall $\left.\Delta_{s}=X_{s}-X_{s-}\right)$

$$
\kappa_{t}(d r)=\rho_{t}(d r)+\eta_{t}(d r)=\sum_{\substack{0<s \leq t \\ X_{s-}<I_{s}^{t}}} \Delta_{s} \delta_{H_{s}}(d r) .
$$

We recall the Poisson representation of $(\rho, \eta)$ under $\mathbb{N}$. Let $\mathcal{N}(d x d \ell d u)$ be a Poisson point measure on $[0,+\infty)^{3}$ with intensity

$$
d x \ell \pi(d \ell) \mathbf{1}_{[0,1]}(u) d u .
$$


For every $a>0$, let us denote by $\mathbb{M}_{a}$ the law of the pair $\left(\mu_{a}, \nu_{a}\right)$ of finite measures on $\mathbb{R}_{+}$ defined by: for $f \in \mathcal{B}_{+}\left(\mathbb{R}_{+}\right)$

$$
\begin{aligned}
\left\langle\mu_{a}, f\right\rangle & =\int \mathcal{N}(d x d \ell d u) \mathbf{1}_{[0, a]}(x) u \ell f(x), \\
\left\langle\nu_{a}, f\right\rangle & =\int \mathcal{N}(d x d \ell d u) \mathbf{1}_{[0, a]}(x) \ell(1-u) f(x) .
\end{aligned}
$$

We eventually set $\mathbb{M}=\int_{0}^{+\infty} d a \mathrm{e}^{-\alpha_{0} a} \mathbb{M}_{a}$.

Proposition 2.6. For every non-negative measurable function $F$ on $\mathcal{M}_{f}\left(\mathbb{R}_{+}\right)^{2}$,

$$
\mathbb{N}\left[\int_{0}^{\sigma} F\left(\rho_{t}, \eta_{t}\right) d t\right]=\int \mathbb{M}(d \mu d \nu) F(\mu, \nu),
$$

where $\sigma=\inf \left\{s>0 ; \rho_{s}=0\right\}$ denotes the length of the excursion.

We recall Lemma 3.2.2 from [10, we shall use later.

Proposition 2.7. Let $\tau$ be an exponential variable of parameter $\lambda>0$ independent of $X$ defined under the measure $\mathbb{N}$. Then, for every $F \in \mathcal{B}_{+}\left(\mathcal{M}_{f}\left(\mathbb{R}_{+}\right)\right)$, we have

$$
\mathbb{N}\left(F\left(\rho_{\tau}\right) \mathbf{1}_{\tau \leq \sigma}\right)=\lambda \int \mathbb{M}(d \mu d \nu) F(\mu) \mathrm{e}^{-\psi^{-1}(\lambda)\langle\nu, 1\rangle} .
$$

It is easy to deduce from this (see also the beginning of Section 3.2.2. [10]) that for $\lambda>0$

$$
\mathbb{N}\left[1-\mathrm{e}^{-\lambda \sigma}\right]=\psi^{-1}(\lambda)
$$

\section{The LÉvy Poisson snake}

As in 1], we want to construct a Poisson snake in order to cut the Lévy CRT at its nodes. For this, we will construct a consistent family $\left(m^{\theta}=\left(m_{t}^{\theta}, t \geq 0\right), \theta \geq 0\right)$ of measure-valued processes. For fixed $\theta$ and $t, m^{\theta}$ will be a point-measure whose atoms mark the atoms of the measure $\rho_{t}$ and such that the set of atoms of $m^{\theta+\theta^{\prime}}$ contains those of $m^{\theta}$. To achieve this, we attach to each jump of $X$ a Poisson process indexed by $\theta$, with intensity equal to this jump. In fact only the first jump of the Poisson processes will be necessary to build the fragmentation process.

3.1. Definition and properties. Conditionally on $\mathcal{X}=\sum_{s>0} \delta_{s, \Delta_{s}}$, we consider a family $\left(\sum_{u>0} \delta_{V_{s, u}}, s \in \mathcal{J}\right)$ of independent Poisson point measures on $\mathbb{R}_{+}$with respective intensity $\Delta_{s} \mathbf{1}_{\{u>0\}} d u$. We define the $\mathcal{M}\left(\mathbb{R}_{+}^{2}\right)$-valued process $M=\left(M_{t}, t \geq 0\right)$ by

$$
M_{t}(d r, d v)=\sum_{\substack{0<s \leq t \\ X_{s-}<I_{t}^{s}}}\left(I_{t}^{s}-X_{s-}\right)\left(\sum_{u>0} \delta_{V_{s, u}}(d v)\right) \delta_{H_{s}}(d r) .
$$

Remark 3.1. The additional coefficient $I_{t}^{s}-X_{s-}$ is not very important and is only needed for the process $M$ to be right-continuous.

Let $\theta>0$. For $t \geq 0$, notice that

$$
M_{t}\left(\mathbb{R}_{+} \times[0, \theta]\right) \leq \sum_{0<s \leq t} \Delta_{s} \xi_{s}
$$


with $\xi_{s}=$ Card $\left\{u>0 ; V_{s, u} \leq \theta\right\}$. In particular, we have for $T>0$,

$$
\sup _{t \in[0, T]} M_{t}\left(\mathbb{R}_{+} \times[0, \theta]\right) \leq \sum_{0<s \leq T} \Delta_{s} \xi_{s}
$$

Notice the variable $\xi_{s}$ are, conditionally on $\mathcal{X}$, independent and distributed as Poisson random variables with parameter $\theta \Delta_{s}$. We have $\mathbb{E}\left[\sum_{0<s \leq T} \Delta_{s} \xi_{s} \mid \mathcal{X}\right]=\theta \sum_{0<s \leq T} \Delta_{s}^{2}$. As $\int_{(0, \infty)}\left(\ell^{2} \wedge\right.$ $\ell) \pi(d \ell)$ is finite, this implies the quantity $\sum_{0<s \leq T} \Delta_{s}^{2}$ is finite a.s. In particular we have a.s.

$$
\sup _{t \in[0, T]} M_{t}\left(\mathbb{R}_{+} \times[0, \theta]\right)<\infty,
$$

and $M_{t}$ is a $\sigma$-finite measure on $\mathbb{R}_{+}^{2}$. Notice that a.s.

$$
M_{t}(d r, d v)=\rho_{t}(d r) M_{t, r}(d v)
$$

where $M_{t, r}$ is a $\sigma$-finite counting measure on $\mathbb{R}_{+}$.

We call the process $\mathcal{S}=\left(\left(\rho_{t}, M_{t}\right), t \geq 0\right)$ the Lévy Poisson snake started at $\rho_{0}=0, M_{0}=0$. To get the Markov property of the Lévy Poisson snake, we must define the process $\mathcal{S}$ started at any initial value $(\mu, \Pi) \in \mathbb{S}$, where $\mathbb{S}$ is the set of pair $(\mu, \Pi)$ such that $\mu \in \mathcal{M}_{f}\left(\mathbb{R}_{+}\right)$and $\Pi(d r, d v)=\mu(d r) \Pi_{r}(d v), \Pi_{r}$ being $\sigma$-finite measures on $\mathbb{R}_{+}$, such that $\Pi\left(\mathbb{R}_{+} \times[0, \theta]\right)<\infty$ for all $\theta \geq 0$. We set $H_{t}^{\mu}=H\left(k_{-I_{t}} \mu\right)$. Then, we define the process $M^{\mu, \Pi}=\left(M_{t}^{\mu, \Pi}, t \geq 0\right)$ by: for $\varphi \in \mathcal{B}_{+}\left(\mathbb{R}_{+}^{2}\right)$,

$$
\left\langle M_{t}^{\mu, \Pi}, \varphi\right\rangle=\int_{(0, \infty)} \varphi(r, v) k_{-I_{t}} \mu(d r) \Pi_{r}(d v)+\int_{(0, \infty)} \varphi\left(r+H_{t}^{\mu}, v\right) M_{t}(d r, d v) .
$$

We shall write $M$ for $M^{\mu, \Pi}$. By construction and since $\rho$ is an homogeneous Markov process, the Lévy Poisson snake $\mathcal{S}=(\rho, M)$ is an homogeneous Markov process.

We now denote by $\mathbb{P}_{\mu, \Pi}$ the law of the Lévy Poisson snake starting at 0 from $(\mu, \Pi)$, and by $\mathbb{P}_{\mu, \Pi}^{*}$ the law of the Lévy Poisson snake killed when $\rho$ reaches 0 . We deduce from (11), that a.s.

$$
\mathbb{E}_{\mu, \Pi}\left[\sup _{t \in[0, T]} M_{t}\left(\mathbb{R}_{+} \times[0, \theta]\right) \mid \mathcal{X}\right] \leq \theta \sum_{0<s \leq T} \Delta_{s}^{2}+\Pi\left(\mathbb{R}_{+} \times[0, \theta]\right)<\infty .
$$

Let $\mathcal{F}=\left(\mathcal{F}_{t}, t \geq 0\right)$ be the filtration generated by $\mathcal{S}$ completed the usual way. Notice this filtration is also generated by the processes $(\mathcal{X}([0, t], \cdot), t \geq 0)$ and $\left(\sum_{s \in \mathcal{J}, s \leq t} \sum_{u \geq 0} \delta_{V_{s, u}}, t \geq\right.$ 0 ). In particular the filtration $\mathcal{F}$ is right continuous. And by construction, we have that $\rho$ is Markovian with respect to $\mathcal{F}$.

Proposition 3.2. The Lévy Poisson snake, $\mathcal{S}$, is a càd-làg strong Markov process in $\mathbb{S} \subset$ $\mathcal{M}_{f}\left(\mathbb{R}_{+}\right) \times \mathcal{M}\left(\mathbb{R}_{+}^{2}\right)$.

Proof. We first check the process $M$ is right continuous. Recall (12). We have by construction a.s. for all $t^{\prime}>t$

$$
M_{t^{\prime}}(d r, d v)=k_{-I_{t^{\prime}}^{t}} \rho_{t}(d r) M_{t, r}(d v)+\rho_{t^{\prime}}(d r) \mathbf{1}_{\left\{r>H_{t, t^{\prime}}\right\}} M_{t^{\prime}, r}(d v),
$$

where $H_{t, t^{\prime}}$ is defined by (3). Thanks to (11), we have, for $\theta>0$,

$$
\int_{\mathbb{R}_{+}} \rho_{t^{\prime}}(d r) \mathbf{1}_{\left\{r>H_{t, t^{\prime}}\right\}} M_{t^{\prime}, r}([0, \theta]) \leq \sum_{t<s \leq t^{\prime}} \Delta_{s} \xi_{s} .
$$


In particular this quantity decreases to 0 as $t^{\prime} \downarrow t$ a.s. By the properties of the exploration process, we recall that a.s. $k_{-I_{t^{\prime}}^{t}} \rho_{t}=\rho_{t^{\prime \prime}}$, where $t^{\prime \prime}=\inf \left\{s \in\left[t, t^{\prime}\right] ; I_{s}^{t}=I_{t^{\prime}}^{t}\right\}$. From the right continuity of $\rho$, we deduce that a.s. for the vague convergence

$$
\lim _{t^{\prime} \downarrow t} M_{t^{\prime}}=M_{t} .
$$

This implies the right continuity of the process $M$ for the vague topology on $\mathcal{M}\left(\mathbb{R}_{+}^{2}\right)$.

Now, we check the process $M$ has left limits. Let $t<t^{\prime}$. For $r \in\left[0, H_{t, t^{\prime}}\right]$, we have $k_{-I_{t^{\prime}}^{t}} \rho_{t}(d r) M_{t, r}=\mathbf{1}_{\left\{r \leq H_{t, t^{\prime}}\right\}} \rho_{t^{\prime}}(d r) M_{t^{\prime}, r}$, as well as

$$
M_{t}(d r, d v)=\mathbf{1}_{\left\{r \leq H_{t, t^{\prime}}\right\}} \rho_{t^{\prime}}(d r) M_{t^{\prime}, r}(d v)+\left[\rho_{t}(d r)-k_{-I_{t^{\prime}}^{t}} \rho_{t}(d r)\right] M_{t, r}(d v) .
$$

If $\rho$ is continuous at $t^{\prime}$, then either $\rho_{t^{\prime}}\left(\left\{H_{t}^{\prime}\right\}\right)=0$ or $H_{t, t^{\prime}}=H_{t^{\prime}}$ for $t$ close enough to $t^{\prime}$. In particular, since $\lim _{t \rightarrow t^{\prime}} H_{t, t^{\prime}}=H_{t^{\prime}}$, we have $\lim _{t \uparrow t^{\prime}} \mathbf{1}_{\left\{r \leq H_{t, t^{\prime}}\right\}} \rho_{t^{\prime}}(d r)=\rho_{t^{\prime}}(d r)$. If $\rho$ is not continuous at $t^{\prime}$, this implies that $\rho_{t^{\prime}}(d r)=\rho_{t^{\prime}-}(d r)+\Delta_{t^{\prime}} \delta_{H_{t^{\prime}}}(d r)$ and for $t$ close enough to $t^{\prime}, H_{t, t^{\prime}}<H_{t^{\prime}}$. Then, we get $\lim _{t \uparrow t^{\prime}} \mathbf{1}_{\left\{r \leq H_{t, t^{\prime}}\right\}} \rho_{t^{\prime}}(d r)=\rho_{t^{\prime}-}(d r)$. In any case, we have a.s. for the vague convergence

$$
\lim _{t \uparrow t^{\prime}} \mathbf{1}_{\left\{r \leq H_{t, t^{\prime}}\right\}} \rho_{t^{\prime}}(d r) M_{t^{\prime}, r}(d v)=\rho_{t^{\prime}-}(d r) M_{t^{\prime}, r}(d v) .
$$

Now, we check that for the vague topology

$$
\lim _{t \uparrow t^{\prime}}\left[\rho_{t}(d r)-k_{-I_{t^{\prime}}^{t}} \rho_{t}(d r)\right] M_{t, r}(d v)=0 .
$$

For this purpose, we remark that

$$
\begin{aligned}
& \mathbb{E}_{\mu, \Pi}\left[\int_{\mathbb{R}_{+}}\left[\rho_{t}(d r)-k_{-I_{t^{\prime}}^{t}} \rho_{t}(d r)\right] M_{t, r}([0, \theta]) \mid \mathcal{X}\right]=\theta \int_{\mathbb{R}_{+}}\left[\rho_{t}(d r)-k_{-I_{t^{\prime}}^{t}} \rho_{t}(d r)\right]\left(\rho_{t}(\{r\})+\eta_{t}(\{r\})\right) \\
& \leq \theta\left(\left\langle\rho_{t}+\eta_{t}, 1\right\rangle\right) \int_{\mathbb{R}_{+}}\left[\rho_{t}(d r)-k_{-I_{t^{\prime}}^{t}} \rho_{t}(d r)\right] \\
& =\theta\left(\left\langle\rho_{t}+\eta_{t}, 1\right\rangle\right)\left(-I_{t^{\prime}}^{t}\right) .
\end{aligned}
$$

As $\rho$ and $\eta$ are respectively càd-làg and càg-làd process, they are bounded over any finite interval a.s. Since $\lim _{t \uparrow t^{\prime}} I_{t^{\prime}}^{t}=0$, we deduce that

$$
\lim _{t \uparrow t^{\prime}} \mathbb{E}_{\mu, \Pi}\left[\int_{\mathbb{R}_{+}}\left[\rho_{t}(d r)-k_{-I_{t^{\prime}}^{t}} \rho_{t}(d r)\right] M_{t, r}([0, \theta]) \mid \mathcal{X}\right]=0 .
$$

Thanks to (13) and Fatou's Lemma, we deduce that

$$
\lim _{t \uparrow t^{\prime}} \int_{\mathbb{R}_{+}}\left[\rho_{t}(d r)-k_{-I_{t^{\prime}}^{t}} \rho_{t}(d r)\right] M_{t, r}([0, \theta])=0 .
$$

Therefore, we conclude that for vague topology,

$$
\lim _{t \uparrow t^{\prime}} M_{t}=M_{t^{\prime}-} .
$$

We deduce that for the vague topology on $\mathcal{M}\left(\mathbb{R}_{+}^{2}\right)$, the process $M$ is a.s. càd-làg. This implies the process $\mathcal{S}$ is a.s. càd-làg.

We check the strong Markov property of $\mathcal{S}$. Mimicking the proof of Proposition 1.2.3 in [10], and using properties of Poisson point measure, one gets that, for any $\mathcal{F}$-stopping time 
$T$, we have a.s. for every $t>0$,

$$
\begin{aligned}
\rho_{T+t} & =\left[k_{-I_{t}^{(T)}} \rho_{T}, \rho_{t}^{(T)}\right] \\
M_{T+t}(d r, d v) & =k_{-I_{t}^{(T)}} \rho_{t}^{(T)}(d r) M_{T, r}(d v)+M_{t}^{(T)}\left(d r+H\left(k_{-I_{t}^{(T)}} \rho_{T}\right), d v\right)
\end{aligned}
$$

where $I^{(T)}, \rho^{(T)}$ and $M^{(T)}$ are the analogues of $I, \rho$ and $M$ with $X$ replaced by the shifted process $X^{(T)}=\left(X_{T+t}-X_{T}, t \geq 0\right)$. This implies the strong Markov property.

3.2. Poisson representation of the snake. Notice that a.s. $\left(\rho_{t}, M_{t}\right)=(0,0)$ if and only if $\rho_{t}=0$. In particular, $(0,0)$ is a regular point for the Lévy Poisson snake, with associated local time $\left(L_{s}^{0}, s \geq 0\right)$. We still write $\mathbb{N}$ for the excursion measure of the Lévy Poisson snake out of $(0,0)$, with the same normalization as in Section 2.4 .

We decompose the path of $\mathcal{S}$ under $\mathbb{P}_{\mu, \Pi}^{*}$ according to excursions of the total mass of $\rho$ above its minimum, see Section 4.2 .3 in [10]. More precisely let $\left(\alpha_{i}, \beta_{i}\right), i \in I$ be the excursion intervals of the process $\langle\rho, 1\rangle$ above its minimum under $\mathbb{P}_{\mu, \Pi}^{*}$. For every $i \in I$, we define $h_{i}=H_{\alpha_{i}}$ and $\mathcal{S}^{i}=\left(\rho^{i}, M^{i}\right)$ by the formulas

$$
\begin{aligned}
\left\langle\rho_{t}^{i}, f\right\rangle & =\int_{\left(h_{i},+\infty\right)} f\left(x-h_{i}\right) \rho_{\left(\alpha_{i}+t\right) \wedge \beta_{i}}(d x) \\
\left\langle M_{t}^{i}, \varphi\right\rangle & =\int_{\left(h_{i},+\infty\right) \times[0,+\infty)} \varphi\left(x-h_{i}, v\right) M_{\left(\alpha_{i}+t\right) \wedge \beta_{i}}(d x, d v) .
\end{aligned}
$$

It is easy to adapt Lemma 4.2.4. of [10] to get the following Lemma.

Lemma 3.3. Let $(\mu, \Pi) \in \mathcal{M}_{f}\left(\mathbb{R}_{+}\right) \times \mathcal{M}\left(\mathbb{R}_{+}^{2}\right)$. The point measure $\sum_{i \in I} \delta_{\left(h_{i}, \mathcal{S}^{i}\right)}$ is under $\mathbb{P}_{\mu, \Pi}^{*}$ a Poisson point measure with intensity $\mu(d r) \mathbb{N}[d \mathcal{S}]$.

3.3. The process $m^{(\theta)}$. For $\theta \geq 0$, we define the $\mathcal{M}\left(\mathbb{R}_{+}\right)$-valued process $m^{(\theta)}=\left(m_{t}^{(\theta)}, t \geq 0\right)$ by

$$
m_{t}^{(\theta)}(d r)=M_{t}(d r,(0, \theta]) .
$$

We make two remarks. We have for $s>0$,

$$
\mathbb{P}_{0,0}\left(m_{s}^{(\theta)}=0 \mid \mathcal{X}\right)=\mathrm{e}^{-\theta \sum_{0<r \leq s, X_{s-}<I_{t}^{s} \Delta_{s}}}=\mathrm{e}^{-\theta\left\langle\kappa_{s}, 1\right\rangle} .
$$

Notice that for $s \in \mathcal{J}$, i.e. $\Delta_{s}>0$, we have $M_{s}\left(\left\{H_{s}\right\}, d v\right)=\Delta_{s} \sum_{u \geq 0} \delta_{V_{s, u}}(d v)$, where conditionally on $\mathcal{X}, \sum_{u \geq 0} \delta_{V_{s, u}}(d v)$ is a Poisson point measure with intensity $\Delta_{s} d u$. In particular, we have

$$
\mathbb{P}_{\mu, \Pi}\left(m_{s}^{(\theta)}\left(\left\{H_{s}\right\}\right)>0 \mid \mathcal{X}\right)=\mathbb{P}\left(M_{s}\left(\left\{H_{s}\right\} \times(0, \theta]\right)>0 \mid \mathcal{X}\right)=1-\mathrm{e}^{-\theta \Delta_{s}} .
$$

From Poisson point measure properties, we get the following Lemma.

Lemma 3.4. The pruned random measure $\mathcal{X}^{\theta}=\sum_{s \geq 0} \mathbf{1}_{\left\{m_{s}^{(\theta)}\left(\left\{H_{s}\right\}\right)>0\right\}} \delta_{s, \Delta_{s}}$ is a Poisson point process with intensity

$$
n^{\theta}(d \ell)=\left(1-\mathrm{e}^{-\theta \ell}\right) \pi(d \ell) .
$$

We shall use later the following property, which is a consequence of Poisson point measure properties. 
Proposition 3.5. Let $M^{\theta}=\left(M_{t}^{\theta}, t \geq 0\right)$ be the measure-valued process defined by

$$
M_{t}^{\theta}(d r,[0, a])=M_{t}(d r,(\theta, \theta+a]), \quad \text { for all } a \geq 0 .
$$

Then, given $\rho, M^{\theta}$ is independent of $M \mathbf{1}_{\mathbb{R}_{+} \times[0, \theta]}$ and is distributed as $M$.

Eventually, the next Lemma on time reversibility can easily be deduced from Corollary 3.1.6 of [10] and the construction of $M$.

Lemma 3.6. Under $\mathbb{N}$, the processes $\left(\left(\rho_{s}, \eta_{s}, \mathbf{1}_{\left\{m_{s}^{(\theta)}=0\right\}}\right), s \in[0, \sigma]\right)$ and $\left(\left(\eta_{(\sigma-s)-}, \rho_{(\sigma-s)-}\right.\right.$, $\left.\left.\mathbf{1}_{\left\{m_{(\sigma-s)-}^{(\theta)}=0\right\}}\right), s \in[0, \sigma]\right)$ have the same distribution.

\section{The PRUned EXPloration PROCESS}

Let us fix $\theta>0$. We shall write $m$ for the process $m^{(\theta)}$ defined in the previous Section. We define the following continuous additive functional of the process $\left(\left(\rho_{t}, m_{t}\right), t \geq 0\right)$ : for $t \geq 0$

$$
A_{t}=\int_{0}^{t} \mathbf{1}_{\left\{m_{s}=0\right\}} d s,
$$

and $C_{t}=\inf \left\{r>0 ; A_{r}>t\right\}$ its right continuous inverse, with the convention that $\inf \emptyset=\infty$. Notice $C_{t}$ is a $\mathcal{F}$-stopping time for any $t \geq 0$ and is finite a.s. from Corollary 4.2 below.

We define the pruned exploration process $\tilde{\rho}=\left(\tilde{\rho}_{t}=\rho_{C_{t}}, t \geq 0\right)$ and the pruned Lévy Poisson snake $\tilde{\mathcal{S}}=(\tilde{\rho}, \tilde{M})$, where $\tilde{M}=\left(M_{C_{t}}, t \geq 0\right)$. In particular the law of $\tilde{M}$ knowing $\tilde{\rho}$ is the law of $M$ knowing $\rho=\tilde{\rho}$. Notice the process $\tilde{\rho}$, and thus the process $\tilde{\mathcal{S}}$, is càd-làg. We also set $\tilde{H}_{t}=H_{C_{t}}$. Let $\tilde{\mathcal{F}}=\left(\tilde{\mathcal{F}}_{t}, t \geq 0\right)$ be the filtration generated by the pruned exploration process $\tilde{\mathcal{S}}$ completed the usual way. In particular $\tilde{\mathcal{F}}_{t} \subset \mathcal{F}_{C_{t}}$, where if $\tau$ is an $\mathcal{F}$-stopping time, then $\mathcal{F}_{\tau}$ is the $\sigma$-field associated to $\tau$.

We introduce the following Laplace exponent $\psi^{(\theta)}$ defined for $\lambda \geq 0$ by

$$
\psi^{(\theta)}(\lambda)=\psi(\lambda+\theta)-\psi(\theta) .
$$

Lemma 4.1. We have the following properties.

(i) For $\lambda>0, \mathbb{N}\left[1-\mathrm{e}^{-\lambda A_{\sigma}}\right]=\psi^{(\theta)^{-1}}(\lambda)$.

(ii) $\mathbb{N}$-a.e. 0 and $\sigma$ are points of increase for $A$. More precisely, $\mathbb{N}$-a.e. for all $\varepsilon>0$, we have $A_{\varepsilon}>0$ and $A_{\sigma}-A_{(\sigma-\varepsilon) \vee 0}>0$.

(iii) $\mathbb{N}$-a.e. the set $\left\{s ; m_{s} \neq 0\right\}$ is dense in $[0, \sigma]$.

Before going into the proof of this Lemma, let us state two direct consequences. From excursion decomposition, see Lemma 3.3. the second part of Lemma 4.1 implies the following corollary.

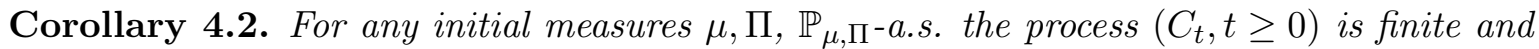
starts at 0 if $m_{0}=0$.

We define $\tilde{\sigma}=\inf \left\{t>0 ; \tilde{\rho}_{t}=0\right\}$. From the second part of Lemma 4.1, we get that $\sigma=\inf \left\{t>0 ; \rho_{t}=0\right\}$ is a left increasing point of $A\left(\mathbb{N}\right.$-a.e. or $\mathbb{P}_{(\mu, \Pi)}$-a.s., $\left.\mu \neq 0\right)$. Therefore, we have $\lim _{r \uparrow A_{\sigma}} C_{r}=\sigma$. As $\rho$ is left continuous at $\sigma$, we get that $\lim _{r \uparrow A_{\sigma}} \tilde{\rho}_{r}=0$ which implies that $\tilde{\sigma} \leq A_{\sigma}$. Since $\tilde{\sigma} \geq A_{\sigma}$, we get that $\mathbb{N}$-a.e.

$$
\tilde{\sigma}=A_{\sigma} .
$$

This equality holds also $\mathbb{P}_{(\mu, \Pi)}$-a.s., for $\mu \neq 0$. 
Proof of Lemma 4.1. We first prove (i). Let $\lambda>0$. Before computing $v=\mathbb{N}\left[1-\exp -\lambda A_{\sigma}\right]$, notice that $A_{\sigma} \leq \sigma$ implies, thanks to (9), that $v \leq \mathbb{N}[1-\exp -\lambda \sigma]=\psi^{-1}(\lambda)<+\infty$. We have

$$
v=\lambda \mathbb{N}\left[\int_{0}^{\sigma} d A_{t} \mathrm{e}^{-\lambda \int_{t}^{\sigma} d A_{u}}\right]=\lambda \mathbb{N}\left[\int_{0}^{\sigma} d A_{t} \mathbb{E}_{\rho_{t}, 0}^{*}\left[\mathrm{e}^{-\lambda A_{\sigma}}\right]\right],
$$

where we replaced $\mathrm{e}^{-\lambda \int_{t}^{\sigma} d A_{u}}$ in the last equality by $\mathbb{E}_{\rho_{t}, 0}^{*}\left[\mathrm{e}^{-\lambda A_{\sigma}}\right]$, its optional projection. In order to compute this last expression, we use the decomposition of $\mathcal{S}$ under $\mathbb{P}_{\mu, \Pi}^{*}$ according to excursions of the total mass of $\rho$ above its minimum, see Lemma 3.3. Using the same notations as in this Lemma, notice that under $\mathbb{P}_{\mu, 0}^{*}$, we have $A_{\sigma}=A_{\infty}=\sum_{i \in I} A_{\infty}^{i}$, with

$$
A_{T}^{i}=\int_{0}^{T} \mathbf{1}_{\left\{M_{t}^{i}\left(\mathbb{R}_{+} \times[0, \theta]\right)=0\right\}} d t .
$$

By Lemma 3.3, we get

$$
\mathbb{E}_{\mu, 0}^{*}\left[\mathrm{e}^{-\lambda A_{\sigma}}\right]=\mathrm{e}^{-\langle\mu, 1\rangle \mathbb{N}\left[1-\exp -\lambda A_{\sigma}\right]}=\mathrm{e}^{-v\langle\mu, 1\rangle} .
$$

Now, for fixed $t$, recall (15). By conditioning with respect to $\mathcal{X}$ or to $\rho$ thanks to Remark 2.5. we have

$$
v=\lambda \mathbb{N}\left[\int_{0}^{\sigma} d A_{t} \mathrm{e}^{-v\left\langle\rho_{t}, 1\right\rangle}\right]=\lambda \mathbb{N}\left[\int_{0}^{\sigma} d t \mathbf{1}_{\left\{m_{t}=0\right\}} \mathrm{e}^{-v\left\langle\rho_{t}, 1\right\rangle}\right]=\lambda \mathbb{N}\left[\int_{0}^{\sigma} d t \mathrm{e}^{-(v+\theta)\left\langle\rho_{t}, 1\right\rangle-\theta\left\langle\eta_{t}, 1\right\rangle}\right] .
$$

Now we use Proposition 2.6 to get

$$
\begin{aligned}
v & =\lambda \int_{0}^{+\infty} d a \mathrm{e}^{-\alpha_{0} a} \mathbb{M}_{a}\left[\mathrm{e}^{-(v+\theta)\langle\mu, 1\rangle-\theta\langle\nu, 1\rangle}\right] \\
& =\lambda \int_{0}^{+\infty} d a \mathrm{e}^{-\alpha_{0} a} \exp \left\{-\int_{0}^{a} d x \int_{0}^{1} d u \int_{(0, \infty)} \ell \pi(d \ell)\left[1-\mathrm{e}^{-(v+\theta) u \ell-\theta(1-u) \ell]}\right\}\right. \\
& =\lambda \int_{0}^{+\infty} d a \exp \left\{-a \int_{0}^{1} d u \psi^{\prime}(\theta+v u)\right\} \\
& =\lambda \frac{v}{\psi(\theta+v)-\psi(\theta)} .
\end{aligned}
$$

where, for the third equality, we used

$$
\psi^{\prime}(\lambda)=\alpha_{0}+\int_{(0, \infty)} \pi(d \ell) \ell\left(1-\mathrm{e}^{-\lambda \ell}\right) .
$$

Notice that if $v=0$, then (20) implies $v=\lambda / \psi^{\prime}(\theta)$, which is absurd. Therefore we have $v \in(0, \infty)$, and we can divide (21) by $v$ to get $\psi^{(\theta)}(v)=\lambda$. This proves (i).

Now, we prove (ii). If we let $\lambda \rightarrow \infty$ in (i) and use that $\lim _{r \rightarrow \infty} \psi^{(\theta)}(r)=+\infty$, then we get that $\mathbb{N}\left[A_{\sigma}>0\right]=+\infty$. Notice that for $(\mu, \Pi) \in \mathbb{S}$, we have under $\mathbb{P}_{\mu, \Pi}^{*}, A_{\infty}=\sum_{i \in I} A_{\infty}^{i}$,

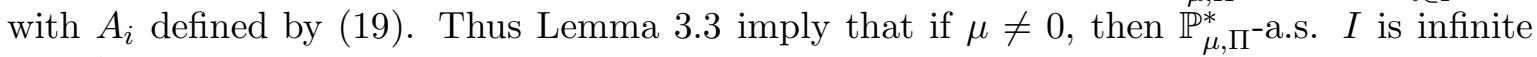
and $A_{\infty}>0$. Using the Markov property at time $t$ of the snake under $\mathbb{N}$, we get that for any $t>0, \mathbb{N}$-a.e. on $\{\sigma>t\}$, we have $A_{\sigma}-A_{t}>0$. This implies that $\sigma$ is a point of increase of $A \mathbb{N}$-a.e. By time reversibility, see Lemma 3.6, we also get that 0 is a point of increase of $A$ $\mathbb{N}$-a.e.

To prove (iii), recall that $\int_{(0,1)} \ell \pi(d \ell)=+\infty$ implies that $\mathcal{J}=\left\{s \geq 0 ; \Delta_{s}>0\right\}$ is dense in $\mathbb{R}_{+}$a.s. Moreover, for every $t>r \geq 0$,

$$
\sum_{r \leq s \leq t} \Delta_{s}=+\infty \text { a.s. }
$$


Now, by the properties of Poisson point measures, we have

$$
\mathbb{P}\left(\forall s \in[r, t], m_{s}=0\right)=\mathbb{E}\left[\mathrm{e}^{-\sum_{r \leq s \leq t} \Delta_{s}}\right]=0
$$

which proves (iii).

\section{A special Markov property}

Let us fix $\theta>0$ and use the notations of the previous Section.

In order to define the excursion of the Lévy Poisson snake out of $\left\{s \geq 0 ; m_{s}=0\right\}$, we define $O$ as the interior of $\left\{s \geq 0, m_{s} \neq 0\right\}$.

Lemma 5.1. $\mathbb{N}$-a.e. the open set $O$ is non empty.

Proof. Thanks to Lemma 4.1 (iii), $\left\{s \geq 0, m_{s} \neq 0\right\}$ is non empty. For any element, $s$, of this set, there exists $u \leq H_{s}$ such that $m_{s}([0, u]) \neq 0$ and $\rho_{s}(\{u\})>0$. Then we consider $\tau_{s}=\inf \left\{t>s, \rho_{t}(\{u\})=0\right\}$. By the right continuity of $\rho, \tau_{s}>s$ and clearly $\left(s, \tau_{s}\right) \subset O$ $\mathbb{N}$-a.e. Therefore $O$ in non empty.

We write $O=\bigcup_{i \in I}\left(\alpha_{i}, \beta_{i}\right)$ and say that $\left(\alpha_{i}, \beta_{i}\right)_{i \in I}$ are the excursions intervals of the Lévy Poisson snake $\mathcal{S}=(\rho, M)$ out of $\left\{s \geq 0, m_{s}=0\right\}$.

Next we prove a special Markov property out of $\left\{s \geq 0, m_{s}=0\right\}$ under the excursion measure $\mathbb{N}$. Using the right continuity of $\rho$ and the definition of $M$, we get that for $i \in I$, $\alpha_{i}>0, \alpha_{i} \in \mathcal{J}$, that is $\rho_{\alpha_{i}}\left(\left\{H_{\alpha_{i}}\right\}\right)=\Delta_{\alpha_{i}}$, and $M_{\alpha_{i}}\left(\left[0, H_{\alpha_{i}}\right),[0, \theta]\right)=0$. For every $i \in I$, let us define the measure-valued process $\mathcal{S}^{i}=\left(\rho^{i}, M^{i}\right)$ by: for every $f \in \mathcal{B}_{+}\left(\mathbb{R}_{+}\right), \varphi \in \mathcal{B}_{+}\left(\mathbb{R}_{+}^{2}\right)$, $t \geq 0$

$$
\begin{aligned}
\left\langle\rho_{t}^{i}, f\right\rangle & =\int_{\left[H_{\alpha_{i}},+\infty\right)} f\left(x-H_{\alpha_{i}}\right) \rho_{\left(\alpha_{i}+t\right) \wedge \beta_{i}}(d x) \\
\left\langle M_{t}^{i}, \varphi\right\rangle & =\int_{\left(H_{\alpha_{i}},+\infty\right) \times[0,+\infty)} \varphi\left(x-H_{\alpha_{i}}, v\right) M_{\left(\alpha_{i}+t\right) \wedge \beta_{i}}(d x, d v) .
\end{aligned}
$$

Since $\rho_{0}^{i}=\delta_{\Delta_{\alpha_{i}}}$, with $\Delta_{\alpha_{i}}>0$, then for every $t<\beta_{i}-\alpha_{i}$, the measure $\rho_{t}^{i}$ charges 0 . As $M_{0}^{i}=0$ we have for every $t<\beta_{i}-\alpha_{i}, M_{t}^{i}\left(\{0\} \times \mathbb{R}_{+}\right)=0$. We call $\Delta_{\alpha_{i}}$ the starting mass of $\mathcal{S}^{i}$

Recall $\tilde{\mathcal{F}}_{\infty}$ is the $\sigma$-field generated by $\tilde{\mathcal{S}}=\left(\left(\rho_{C_{t}}, M_{C_{t}}\right), t \geq 0\right)$ and $\mathbb{P}_{\mu, \Pi}^{*}(d \mathcal{S})$ denotes the law of the snake $\mathcal{S}$ started at $(\mu, \Pi)$ and stopped when $\rho$ reaches 0 . For $\ell \in[0,+\infty)$, we will write $\mathbb{P}_{\ell}^{*}$ for $\mathbb{P}_{\delta_{\ell}, 0}^{*}$. Recall (16) and define the measure $\mathrm{N}$ by

$$
\mathrm{N}(d \mathcal{S})=\int_{(0,+\infty)} \pi(d \ell)\left(1-\mathrm{e}^{-\theta \ell}\right) \mathbb{P}_{\ell}^{*}(d \mathcal{S})=\int_{(0, \infty)} n^{(\theta)}(d \ell) \mathbb{P}_{\ell}^{*}(d \mathcal{S})
$$

If $Q$ is a measure on $\mathbb{S}$ and $\phi$ is a non-negative measurable function defined on a measurable space $\mathbb{R}_{+} \times \Omega \times \mathbb{S}$, we denote by

$$
Q[\phi(u, \omega, \cdot)]=\int_{\mathbb{S}} \phi(u, \omega, \mathcal{S}) Q(d \mathcal{S})
$$

In other words, the integration concerns only the third component of the function $\phi$.

Recall the definition of $\tilde{\sigma}$ given after Corollary 4.2 
Theorem 5.2. (Special Markov property) Let $\phi$ be a non-negative measurable function defined on $\mathbb{R}_{+} \times \Omega \times \mathbb{S}$ such that $t \mapsto \phi(t, \omega, \mathcal{S})$ is progressively $\tilde{\mathcal{F}}$-measurable for any $\mathcal{S} \in \mathbb{S}$. Then, we have $\mathbb{N}$-a.e.

$$
\mathbb{N}\left[\exp \left(-\sum_{i \in I} \phi\left(A_{\alpha_{i}}, \omega, \mathcal{S}^{i}\right)\right) \mid \tilde{\mathcal{F}}_{\infty}\right]=\exp \left(-\int_{0}^{\tilde{\sigma}} d u \mathrm{~N}\left[1-\mathrm{e}^{-\phi(u, \omega, \cdot)}\right]\right) .
$$

In particular, the law of the excursion process $\sum_{i \in I} \delta_{\left(A_{\alpha_{i}}, \mathcal{S}^{i}\right)}$, given $\tilde{\mathcal{F}}_{\infty}$ under $\mathbb{N}$, is the law of a Poisson point measure of intensity $\mathbf{1}_{[0, \tilde{\sigma}]}(u) d u \mathrm{~N}(d \mathcal{S})$.

Before going into the proof of this Theorem, we give a corollary we shall use later.

Corollary 5.3. The law of the excursion process $\sum_{i \in I} \delta_{\left(A_{\alpha_{i}}, \rho_{\alpha_{i}-}, \mathcal{S}^{i}\right)}$, given $\tilde{\mathcal{F}}_{\infty}$, is the law of a Poisson point measure of intensity $\mathbf{1}_{[0, \tilde{\sigma}]}(u) d u \delta_{\tilde{\rho}_{u}}(d \mu) \mathrm{N}(d \mathcal{S})$.

Proof. This is a direct consequence of Theorem 5.2 and Lemma 5.5 .

The rest of this Section is devoted to the proof of the special Markov property.

5.1. A remark and notations. To begin with, let us remark that to prove Theorem 5.2 , we may only consider function $\phi$ satisfying the hypothesis of Theorem 5.2 and those two conditions:

$\left(h_{1}\right) \phi(s, \omega, \mathcal{S})=0$ if the starting mass of $\mathcal{S}$ is less than $\eta$, that is $\left\langle\rho_{0}, 1\right\rangle \leq \eta$, for a fixed positive real number $\eta$.

$\left(h_{2}\right) t \mapsto \phi(t, \omega, \mathcal{S})$ is continuous for all $\mathcal{S} \in \mathbb{S}$ a.s.

Indeed if (24) holds for such functions then by monotone class Theorem and monotonicity it holds also for every function satisfying the hypothesis of Theorem 5.2. From now on, but for Lemma 5.5. we fix $\eta>0$, and we assume the function $\phi$ satisfies the hypothesis of Theorem 5.2 and $\left(h_{1}\right)$. We will assume $\left(h_{2}\right)$ only for Sections 5.6 and 5.7

Let $\varepsilon<\eta$ and let us define by induction (under the measure $\mathbb{N}$ ) the following stopping times: $T_{0}^{\varepsilon}=0$ and, for every integer $k \geq 0$,

$$
\begin{aligned}
& S_{k+1}^{\varepsilon}=\inf \left\{s>T_{k}^{\varepsilon}, m_{s}\left(\left\{H_{s}\right\}\right)>0, \rho_{s}\left(\left\{H_{s}\right\}\right)>\varepsilon\right\} \\
& T_{k+1}^{\varepsilon}=\inf \left\{s>S_{k+1}^{\varepsilon}, m_{s}=0\right\}
\end{aligned}
$$

with the convention $\inf \emptyset=\sigma$. Let us then denote

$$
N_{\varepsilon}=\sup \left\{k \in \mathbb{N}, S_{k}^{\varepsilon} \neq \sigma\right\} .
$$

Notice $N_{\varepsilon}$ is finite $\mathbb{N}$-a.e. as there is a finite number of jumps $\Delta_{t}>\varepsilon$.

For every $k \leq N_{\varepsilon}$, we define the measure-valued process $\mathcal{S}^{k, \varepsilon}=\left(\rho^{k, \varepsilon}, M^{k, \varepsilon}\right)$ in the same way as the processes $\rho^{i}$ and $M^{i}$ : for every non-negative continuous functions $f$ and $\varphi$, and $t \geq 0$

$$
\begin{aligned}
\left\langle\rho_{t}^{k, \varepsilon}, f\right\rangle & =\int_{\left[H_{S_{k}^{\varepsilon}},+\infty\right)} f\left(x-H_{S_{k}^{\varepsilon}}\right) \rho_{\left(S_{k}^{\varepsilon}+t\right) \wedge T_{k}^{\varepsilon}}(d x) \\
\left\langle M_{t}^{k, \varepsilon}, \varphi\right\rangle & =\int_{\left(H_{S_{k}^{\varepsilon}},+\infty\right) \times[0,+\infty)} \varphi\left(x-H_{S_{k}^{\varepsilon}}, v\right) M_{\left(S_{k}^{\varepsilon}+t\right) \wedge T_{k}^{\varepsilon}}(d x, d v) .
\end{aligned}
$$


We call $\Delta_{S_{k}^{\varepsilon}}$ the starting mass of $\mathcal{S}^{k, \varepsilon}$. Notice that $\rho_{0}^{k, \varepsilon}=\delta_{\Delta_{S_{k}^{\varepsilon}}}$ and $\Delta_{S_{k}^{\varepsilon}} \geq \varepsilon$ for $k \in$ $\left\{1, \ldots, N_{\varepsilon}\right\}$. Notice also that $\mathbb{N}$-a.e,

$$
\lim _{\varepsilon \rightarrow 0} \bigcup_{k \in \mathbb{N}}\left(S_{k}^{\varepsilon}, T_{k}^{\varepsilon}\right)=\bigcup_{i \in I}\left(\alpha_{i}, \beta_{i}\right)
$$

\subsection{Approximation of the excursion process.}

Lemma 5.4. $\mathbb{N}$-a.e., we have for $\varepsilon>0$ small enough

$$
\sum_{i \in I} \phi\left(A_{\alpha_{i}}, \omega, \mathcal{S}^{i}\right)=\sum_{k=2}^{N_{\varepsilon}} \phi\left(A_{S_{k}^{\varepsilon}}, \omega, \mathcal{S}^{k, \varepsilon}\right) .
$$

Proof. Let $I_{\eta}$ be the set of indexes $i \in I$, such that the starting mass of $\mathcal{S}^{i}$ is larger than $\eta$. Because of $\left(h_{1}\right)$, we have

$$
\sum_{i \in I} \phi\left(A_{\alpha_{i}}, \omega, \mathcal{S}^{i}\right)=\sum_{i \in I_{\eta}} \phi\left(A_{\alpha_{i}}, \omega, \mathcal{S}^{i}\right)
$$

Let $\varepsilon<\eta$. Then, for any $i \in I_{\eta}$, there exists $k \in \mathbb{N}^{*}$, such that $\mathcal{S}^{k, \varepsilon}=\mathcal{S}^{i}$.

Furthermore, all the others excursions $\mathcal{S}^{k, \varepsilon}$ which don't belong to $\left\{\mathcal{S}^{i}, i \in I_{\eta}\right\}$ either have a starting mass less that $\eta$ (and thus $\phi\left(A_{S_{k}^{\varepsilon}}, \omega, \mathcal{S}^{k, \varepsilon}\right)=0$ ), or have a starting mass greater that $\eta$ but $m_{S_{k}^{\varepsilon}}\left(\left[0, H_{S_{k}^{\varepsilon}}\right)\right)>0$. But, as the set $\left\{s \geq 0, \Delta_{s}>\eta\right\}$ is finite, there exists only a finite number of excursions $\mathcal{S}^{i}$ which straddle a time $s$ such that $\Delta_{s}>\eta$. Therefore, the minimum over those excursions of their starting mass, say $\eta^{\prime}$, is positive a.s. and, if we choose $\varepsilon<\eta^{\prime}$, there are no excursions $\mathcal{S}^{k, \varepsilon}$ with initial mass greater than $\eta$ which do not correspond to a $\mathcal{S}^{i}$

Consequently, if we choose $\varepsilon<\eta \wedge \eta^{\prime}$, we have

$$
\sum_{i \in I} \phi\left(A_{\alpha_{i}}, \omega, \mathcal{S}^{i}\right)=\sum_{k=1}^{N_{\varepsilon}} \phi\left(A_{S_{k}^{\varepsilon}}, \omega, \mathcal{S}^{k, \varepsilon}\right) .
$$

Notice also, that because of Lemma 4.1 (iii), for $\varepsilon>0$ small enough, the starting mass of $\mathcal{S}^{1, \varepsilon}$ is less than $\eta$. Therefore, we deduce that (27) holds $\mathbb{N}$-a.e. for $\varepsilon>0$ small enough.

We can now prove the next Lemma which gives Corollary 5.3 .

Lemma 5.5. Let $\psi$ be a bounded non-negative measurable function defined on $\mathbb{R}_{+} \times \mathcal{M}_{f}\left(\mathbb{R}_{+}\right) \times$ $\mathbb{S}$. $\mathbb{N}$-a.e., we have

$$
\sum_{i \in I} \psi\left(A_{\alpha_{i}}, \rho_{\alpha_{i}-}, \mathcal{S}^{i}\right)=\sum_{i \in I} \phi\left(A_{\alpha_{i}}, \omega, \mathcal{S}^{i}\right)
$$

where $\phi(t, \omega, \mathcal{S})=\psi\left(t, \tilde{\rho}_{t}(\omega), \mathcal{S}\right)$.

Proof. First we assume that $\psi(t, \mu, \mathcal{S})=0$ if the starting mass of $\mathcal{S}$ is less than $\eta$. The same arguments as those used to prove Lemma 5.4 yields that $\mathbb{N}$-a.e. for $\varepsilon>0$ small enough, we have

$$
\sum_{i \in I} \psi\left(A_{\alpha_{i}}, \rho_{\alpha_{i}-}, \mathcal{S}^{i}\right)=\sum_{k=2}^{N_{\varepsilon}} \psi\left(A_{S_{k}^{\varepsilon}}, \rho_{S_{k}^{\varepsilon}-}, \mathcal{S}^{k, \varepsilon}\right) .
$$

Notice that by construction, $\rho_{S_{k}^{\varepsilon}-}=\rho_{T_{k}^{\varepsilon}}$ and that $m_{T_{k}^{\varepsilon}}=0$. Using the strong Markov property at time $T_{k}^{\varepsilon}$ and the second part of Corollary 4.2, we deduce that $\mathbb{N}$-a.e. for all $k \in \mathbb{N}^{*}$,

$$
C_{A_{T_{k}^{\varepsilon}}}=T_{k}^{\varepsilon} .
$$


Therefore, as $A_{S_{k}^{\varepsilon}}=A_{T_{k}^{\varepsilon}}$, we have $\mathbb{N}$-a.e.

$$
\tilde{\rho}_{A_{S_{k}^{\varepsilon}}}=\tilde{\rho}_{A_{T_{k}^{\varepsilon}}}=\rho_{T_{k}^{\varepsilon}}=\rho_{S_{k}^{\varepsilon}-} .
$$

Hence, we have that $\mathbb{N}$-a.e. for $\varepsilon>0$ small enough,

$$
\sum_{i \in I} \psi\left(A_{\alpha_{i}}, \rho_{\alpha_{i}-}, \mathcal{S}^{i}\right)=\sum_{k=2}^{N_{\varepsilon}} \phi\left(A_{S_{k}^{\varepsilon}}, \omega, \mathcal{S}^{k, \varepsilon}\right),
$$

with $\phi(t, \omega, \mathcal{S})=\psi\left(t, \tilde{\rho}_{t}(\omega), \mathcal{S}\right)$. Now, we complete the proof using Lemma 5.4 and letting $\eta \downarrow 0$.

5.3. A measurability result. We shall use later the next additive functional defined for $s \geq 0$ by

$$
A_{s}^{\varepsilon}=\sum_{k=0}^{N_{\varepsilon}} \int_{0}^{s} \mathbf{1}_{\left[T_{k}^{\varepsilon}, S_{k+1}^{\varepsilon}\right]}(u) d u .
$$

For $k \geq 1$, we consider the $\sigma$-field $\mathcal{F}^{(\varepsilon), k}$ generated by the family of processes

$$
\left(\mathcal{S}_{\left(T_{l}^{\varepsilon}+s\right) \wedge S_{l+1}^{\varepsilon}-}, s>0\right)_{l \in\{0, \ldots, k-1\}} .
$$

Notice that for $k \in \mathbb{N}^{*}$

$$
\mathcal{F}^{(\varepsilon), k} \subset \mathcal{F}_{S_{k}^{\varepsilon}}
$$

Lemma 5.6. For any $\varepsilon>0, k \in \mathbb{N}^{*}$, the function $\phi\left(A_{S_{k}^{\varepsilon}}, \omega, \cdot\right)$ is $\mathcal{F}^{(\varepsilon), k}$-measurable.

Proof. We set $C_{s}^{\varepsilon}$ the right continuous inverse of $A_{s}^{\varepsilon}$ and we define the filtration $\tilde{\mathcal{F}}^{(\varepsilon)}=$ $\left(\tilde{\mathcal{F}}_{t}^{(\varepsilon)}, t \geq 0\right)$ generated by the process $\left(\mathcal{S}_{C_{s}^{\varepsilon}}, s \geq 0\right)$.

We consider the counting process $\left(R_{t}, t \geq 0\right)$ defined by $R_{t}=\inf \left\{k \geq 0 ; S_{k+1}^{\varepsilon}>A_{t}^{\varepsilon}\right\}$. Consider the filtration $\mathcal{F}^{(\varepsilon)}=\left(\mathcal{F}_{t}^{(\varepsilon)}, t \geq 0\right)$, where $\mathcal{F}_{t}^{(\varepsilon)}=\tilde{\mathcal{F}}_{t}^{(\varepsilon)} \vee \sigma\left(R_{s}, s \leq t\right)$. In particular for $k \geq 1, A_{S_{k}^{\varepsilon}}^{\varepsilon}=\inf \left\{t \geq 0 ; R_{t}=k\right\}$ is a $\mathcal{F}^{(\varepsilon)}$-stopping time. Notice then that $\mathcal{F}^{(\varepsilon), k}=\mathcal{F}_{A_{S_{k}^{\varepsilon}}^{\varepsilon}}^{(\varepsilon)}$.

By the monotone class Theorem, to prove the Lemma, it is enough to consider simple processes, $\phi$, defined by $\phi(t, \omega, \mathcal{S})=g(\mathcal{S}) Z \mathbf{1}_{\{r \leq t\}}$, where $r \geq 0, Z \in \tilde{\mathcal{F}}_{r}$, and $g$ is a real measurable function defined on $\mathbb{S}$. For $k \in \mathbb{N}^{*}$, we have $\phi\left(A_{S_{k}^{\varepsilon}}, \omega, \cdot\right)=g Z \mathbf{1}_{\left\{r \leq A_{S_{k}}\right\}}$. Notice that

$$
\begin{aligned}
A_{C_{r}}^{\varepsilon} & =\inf \left\{u>0 ; C_{u}^{\varepsilon}>C_{r}\right\} \\
& =\inf \left\{u>0 ; \int_{0}^{C_{u}^{\varepsilon}} \mathbf{1}_{\left\{m_{s}=0\right\}} d s>r\right\} \\
& =\inf \left\{u>0 ; \int_{0}^{C_{u}^{\varepsilon}} \mathbf{1}_{\left\{m_{s}=0\right\}} d A_{s}^{\varepsilon}>r\right\} \\
& =\inf \left\{u>0 ; \int_{0}^{u} \mathbf{1}_{\left\{m_{\left.C_{t}^{\varepsilon}=0\right\}}\right.} d t>r\right\},
\end{aligned}
$$

where we used that $A^{\varepsilon}$ is the right continuous inverse of $C^{\varepsilon}$ for the first equality, $C$ is the right continuous inverse of $A$ for the second, $\left\{s ; m_{s}=0\right\} \subset \bigcup_{k \geq 0}\left[T_{k}^{\varepsilon}, S_{k+1}^{\varepsilon}\right]$ for the third, and 
the change of variable $t=A_{s}^{\varepsilon}$ for the last. This gives that $A_{C_{r}}^{\varepsilon}$ is a $\mathcal{F}^{(\varepsilon)}$-stopping time. By composition of random change time, we also have $\tilde{\mathcal{F}}_{r} \subset \mathcal{F}_{A_{C_{r}}^{\varepsilon}}^{(\varepsilon)}$. Eventually, we have

$$
\left\{r \leq A_{S_{k}^{\varepsilon}}\right\}=\left\{r \leq A_{T_{k}^{\varepsilon}}\right\}=\left\{C_{r} \leq T_{k}^{\varepsilon}\right\}=\left\{A_{C_{r}}^{\varepsilon} \leq A_{T_{k}^{\varepsilon}}^{\varepsilon}\right\}=\left\{A_{C_{r}}^{\varepsilon} \leq A_{S_{k}^{\varepsilon}}^{\varepsilon}\right\}
$$

where we used $A_{S_{k}^{\varepsilon}}=A_{T_{k}^{\varepsilon}}$ for the first equality, (28) and the definition of $C$ for the second, and similar properties for $A^{\varepsilon}$ for the two last ones. We deduce then that $Z \mathbf{1}_{\left\{r \leq A_{S_{k}^{\varepsilon}}\right\}}=$ $Z \mathbf{1}_{\left\{A_{C_{r}}^{\varepsilon} \leq A_{S_{k}^{\varepsilon}}^{\varepsilon}\right\}}$ is measurable with respect to $\mathcal{F}_{A_{S_{k}^{\varepsilon}}^{\varepsilon}}^{(\varepsilon)}=\mathcal{F}^{(\varepsilon), k}$. This ends the proof of the Lemma.

\subsection{Computation of the conditional expectation of the approximation.}

Lemma 5.7. For every $\tilde{\mathcal{F}}_{\infty}$-measurable non-negative random variable $Z$, we have

$$
\mathbb{N}\left[Z \exp \left(-\sum_{k=2}^{N_{\varepsilon}} \phi\left(A_{S_{k}^{\varepsilon}}, \omega, \mathcal{S}^{k, \varepsilon}\right)\right)\right]=\mathbb{N}\left[Z \prod_{k=2}^{N_{\varepsilon}} \mathrm{N}\left[\mathrm{e}^{-\phi\left(A_{S_{k}^{\varepsilon}}, \omega, \cdot\right)} \mid \rho_{0}>\varepsilon\right]\right] .
$$

Proof. For every integer $p \geq 2$, we consider a non-negative random variable $Z$ of the form $Z=Z_{0} Z_{1}$, where $Z_{0} \in \overline{\mathcal{F}}^{(\varepsilon), p}$ and $Z_{1} \in \sigma\left(\mathcal{S}_{\left(T_{k}^{\varepsilon}+s\right) \wedge S_{k+1}^{\varepsilon}-}, s \geq 0, k \geq p\right)$ are bounded non-negative and such that $\mathbb{N}\left[Z_{0}\right]<\infty$.

To compute $\mathbb{N}\left[Z \exp \left(-\sum_{k=2}^{p} \phi\left(A_{S_{k}^{\varepsilon}}, \omega, \mathcal{S}^{k, \varepsilon}\right)\right)\right]$, we first apply the strong Markov property at time $T_{p}^{\varepsilon}$. We obtain

$$
\mathbb{N}\left[Z \exp \left(-\sum_{k=2}^{p} \phi\left(A_{S_{k}^{\varepsilon}}, \omega, \mathcal{S}^{k, \varepsilon}\right)\right)\right]=\mathbb{N}\left[Z_{0} \exp \left(-\sum_{k=2}^{p} \phi\left(A_{S_{k}^{\varepsilon}}, \omega, \mathcal{S}^{k, \varepsilon}\right)\right) \mathbb{E}_{\rho_{T_{p}^{\varepsilon}}^{*}, 0}\left[Z_{1}\right]\right] .
$$

Notice that $\rho_{T_{p}^{\varepsilon}}=\rho_{S_{p}^{\varepsilon}-}$, and consequently $\rho_{T_{p}^{\varepsilon}}$ is measurable with respect to $\mathcal{F}_{S_{p}^{\varepsilon}}$. So, when we use the strong Markov property at time $S_{p}^{\varepsilon}$, we get thanks to Lemma [5.6 and (30),

$$
\begin{aligned}
\mathbb{N}\left[Z \exp \left(-\sum_{k=2}^{p} \phi\left(A_{S_{k}^{\varepsilon}}, \omega, \mathcal{S}^{k, \varepsilon}\right)\right)\right] \\
=\mathbb{N}\left[Z _ { 0 } \operatorname { e x p } \left(-\sum_{k=2}^{p-1} \phi\left(A_{\left.S_{k}^{\varepsilon}, \omega, \mathcal{S}^{k, \varepsilon}\right)}\right) \mathbb{E}_{\rho_{0}^{p, \varepsilon}, 0}^{*}\left[\mathrm{e}^{-\phi\left(A_{\left.S_{p}^{\varepsilon}, \omega, \cdot\right)}\right)} \mathbb{E}_{\rho_{T_{p}^{\varepsilon}, 0}^{*}\left[Z_{1}\right]}\right] .\right.\right.
\end{aligned}
$$

Recall $p \geq 2$. Conditionally on $\mathcal{F}_{T_{p-1}^{\varepsilon}}$, on $N_{\varepsilon} \geq p$, the measure $\rho_{0}^{p, \varepsilon}$ is a Dirac mass and, by the Poisson representation of Lemma 3.4 this mass is the first atom of the Poisson point measure $\mathcal{X}^{\theta}$ that lies in $(\varepsilon,+\infty)$. Consequently, the mass of $\rho_{0}^{p, \varepsilon}$ is distributed according to the law $n^{\theta}(d \ell \mid \ell>\varepsilon)$. From Poisson point measure properties, notice that $\rho_{0}^{p, \varepsilon}$ is also independent of $\sigma\left(\mathcal{S}_{t}, t<S_{p}^{\varepsilon}\right)$ and thus of $\mathcal{F}^{(\varepsilon), p}$. 
Therefore, conditionally on $N_{\varepsilon} \geq p, \rho_{0}^{p, \varepsilon}$ is independent of $Z_{0}, \rho_{T_{p}^{\varepsilon}}=\rho_{S_{p}^{\varepsilon}-}$ and, thanks to Lemma 5.6 of $\phi\left(A_{S_{p}^{\varepsilon}}, \omega, \cdot\right)$. So, by conditioning with respect to $\mathcal{F}^{(\varepsilon), p}$, we get

$$
\begin{aligned}
\mathbb{N}[Z \exp ( & \left.\left.-\sum_{k=2}^{p} \phi\left(A_{S_{k}^{\varepsilon}}, \omega, \mathcal{S}^{k, \varepsilon}\right)\right)\right] \\
& =\mathbb{N}\left[Z_{0} \exp \left(-\sum_{k=2}^{p-1} \phi\left(A_{S_{k}^{\varepsilon}}, \omega, \mathcal{S}^{k, \varepsilon}\right)\right) \mathrm{N}\left[\mathrm{e}^{-\phi\left(A_{\left.S_{p}^{\varepsilon}, \omega, \cdot\right)}\right.} \mid \rho_{0}>\varepsilon\right] \mathbb{E}_{\rho_{T_{p}^{\varepsilon}, 0}^{*}\left[Z_{1}\right]}\right] .
\end{aligned}
$$

Remark 5.8. From point Poisson measure property, notice that, conditionally on $\mathcal{F}_{T_{p-1}^{\varepsilon}}$ and $N_{\varepsilon} \geq p \geq 2, e_{p}^{\varepsilon}=S_{p}^{\varepsilon}-T_{p-1}^{\varepsilon}$ is an exponential random variable with parameter

$$
n_{\varepsilon}=n^{\theta}(\ell>\varepsilon)=\int_{(\varepsilon,+\infty)} \pi(d \ell)\left(1-\mathrm{e}^{-\theta \ell}\right) .
$$

And, conditionally on $N_{\varepsilon}$ and $N_{\varepsilon} \geq 2$, the random variables $\left(e_{k}^{\varepsilon}, k \in\left\{2, \ldots, N_{\varepsilon}\right\}\right)$ are independent exponential random variables with parameter $n_{\varepsilon}$.

Now, using one more time the strong Markov property at time $T_{p}^{\varepsilon}$, we get from (31)

$$
\begin{aligned}
\mathbb{N}\left[Z \exp \left(-\sum_{k=2}^{p} \phi\left(A_{S_{k}^{\varepsilon}}, \omega, \mathcal{S}^{k, \varepsilon}\right)\right)\right] & \\
= & \mathbb{N}\left[Z \mathrm{~N}\left[\mathrm{e}^{-\phi\left(A_{\left.S_{p}^{\varepsilon}, \omega, \cdot\right)}\right.} \mid \rho_{0}>\varepsilon\right] \exp \left(-\sum_{k=2}^{p-1} \phi\left(A_{S_{k}^{\varepsilon}}, \omega, \mathcal{S}^{k, \varepsilon}\right)\right)\right] .
\end{aligned}
$$

From monotone class Theorem, this equality holds also for any $Z \in \mathcal{F}^{(\varepsilon), \infty}$ non-negative. Thanks to Lemma [5.6] the non-negative random variable $Z^{\prime}=Z \mathrm{~N}\left[\mathrm{e}^{-\phi\left(A_{\left.S_{p}^{\varepsilon}, \omega, \cdot\right)}\right.} \mid \rho_{0}>\varepsilon\right]$ is measurable w.r.t. $\mathcal{F}^{(\varepsilon), \infty}$. So, we may iterate the previous argument and eventually get that for any non-negative random variable $Z \in \mathcal{F}^{(\varepsilon), \infty}$, we have

$$
\mathbb{N}\left[Z \exp \left(-\sum_{k=2}^{p} \phi\left(A_{S_{k}^{\varepsilon}}, \omega, \mathcal{S}^{k, \varepsilon}\right)\right)\right]=\mathbb{N}\left[Z \prod_{k=2}^{p} \mathrm{~N}\left[\mathrm{e}^{-\phi\left(A_{S_{k}^{\varepsilon}}, \omega, \cdot\right)} \mid \rho_{0}>\varepsilon\right]\right] .
$$

Let $p \rightarrow+\infty$ and notice that $\tilde{\mathcal{F}}_{\infty} \subset \mathcal{F}^{(\varepsilon), \infty}$ to end the proof.

5.5. An ancillary result. Recall (25) and (32) We prove the next result.

Lemma 5.9. There exists a positive sequence $\left(\varepsilon_{j}, j \in \mathbb{N}^{*}\right)$ decreasing to 0 , such that $\mathbb{N}$-a.e.:

(i) $\lim _{j \rightarrow \infty} \frac{N_{\varepsilon_{j}}}{n_{\varepsilon_{j}}}=A_{\sigma}$.

(ii) For any $g \in \mathcal{B}_{+}\left(\mathbb{R}_{+}\right)$bounded continuous, we have

$$
\lim _{j \rightarrow \infty} \frac{1}{n_{\varepsilon_{j}}} \sum_{k=2}^{N_{\varepsilon_{j}}} g\left(A_{S_{k}^{\varepsilon_{j}}}\right)=\int_{0}^{\tilde{\sigma}} g(u) d u .
$$

Proof. Notice that $\left\{s ; m_{s} \neq 0\right\} \subset O \cup\left\{s ; \Delta_{s} \neq 0\right\}$ (see proof of Lemma 5.1). As $\left\{s ; \Delta_{s} \neq 0\right\}$ is discrete, we have thanks to (26), that $\mathbb{N}$-a.e. for all $s \geq 0, \lim _{\varepsilon \rightarrow 0} A_{s}^{\varepsilon}=A_{s}$ where $A^{\varepsilon}$ is defined by (29). From Dini Theorem this convergence is uniform on $[0, \sigma] \mathbb{N}$-a.e. In particular, (ii) 
will be proved once we proved (i) and that $\mathbb{N}$-a.e. for any $g \in \mathcal{B}_{+}\left(\mathbb{R}_{+}\right)$bounded continuous, we have

$$
\lim _{j \rightarrow \infty} \frac{1}{n_{\varepsilon_{j}}} \sum_{k=2}^{N_{\varepsilon_{j}}} g\left(A_{S_{k}^{\varepsilon_{j}}}^{\varepsilon_{j}}\right)=\int_{0}^{\tilde{\sigma}} g(u) d u .
$$

From Remark 5.8, we see there exists a sequence of random variables $\left(e_{k}^{\varepsilon}, k \geq 2\right)$, such that conditionally on $N_{\varepsilon}$, they are independent exponential variables of parameter $n_{\varepsilon}$ (see (32)) and

$$
A_{\sigma}^{\varepsilon}=\left(S_{1}^{\varepsilon}-T_{0}^{\varepsilon}\right)+\sum_{k=2}^{N_{\varepsilon}} e_{k}^{\varepsilon}+\left(S_{N_{\varepsilon}+1}^{\varepsilon}-T_{N_{\varepsilon}}^{\varepsilon}\right)
$$

We set $e_{0}^{\varepsilon}=S_{N_{\varepsilon}+1}^{\varepsilon}-T_{N_{\varepsilon}}^{\varepsilon}$ and $e_{1}^{\varepsilon}=S_{1}^{\varepsilon}-T_{0}^{\varepsilon}$, so that we have the compact notation $A_{\sigma}^{\varepsilon}=$ $\sum_{k=0}^{N_{\varepsilon}} e_{k}^{\varepsilon}$ and $A_{S_{k}^{\varepsilon}}^{\varepsilon}=\sum_{l=1}^{k} e_{l}^{\varepsilon}$ for $k \leq N_{\varepsilon}$.

Because of Lemma 4.1 (ii) and (iii) we have that $\mathbb{N}$-a.e. $\lim _{\varepsilon \downarrow 0} e_{0}^{\varepsilon}=\lim _{\varepsilon \downarrow 0} e_{1}^{\varepsilon}=0$. We deduce that $\mathbb{N}$-a.e.

$$
\lim _{\varepsilon \downarrow 0} \sum_{k=2}^{N_{\varepsilon}} e_{k}^{\varepsilon}=\lim _{\varepsilon \downarrow 0} A_{\sigma}^{\varepsilon}=A_{\sigma} .
$$

Conditionally on $N_{\varepsilon}$, the random variables $\left(n_{\varepsilon} e_{k}^{\varepsilon}, k \geq 2\right)$ are independent exponential variables of parameter 1 . The previous equality and the law of large numbers implies that $\mathbb{N}$-a.e. for anypositive deterministic sequence $\left(\varepsilon_{j}, j \in \mathbb{N}\right)$ that decreases to 0 , and we obtain (i).

To get (133), we choose the sequence $\left(\varepsilon_{j}, j \in \mathbb{N}\right)$ so that for some $\delta \in(0,1 / 3)$, we have

$$
\sum_{j=1}^{+\infty} n_{\varepsilon_{j}}^{-(1-3 \delta) / 2}<+\infty
$$

As a consequence of (i), there exists a (random) integer $J$ such that, if $j \geq J$,

$$
N_{\varepsilon_{j}} \leq n_{\varepsilon_{j}}^{1+\delta} .
$$

Notice that to prove (33), it is enough to consider $g$ bounded and Lipschitz. We have for $j \geq J$,

$$
\begin{aligned}
\left|\frac{1}{n_{\varepsilon_{j}}} \sum_{k=2}^{N_{\varepsilon_{j}}} g\left(A_{S_{k}^{\varepsilon_{j}}}^{\varepsilon_{j}}\right)-\frac{1}{n_{\varepsilon_{j}}} \sum_{k=2}^{N_{\varepsilon_{j}}} g\left(\frac{k}{n_{\varepsilon_{j}}}\right)\right| & \leq C_{g} \frac{1}{n_{\varepsilon_{j}}} \sum_{k=2}^{N_{\varepsilon_{j}}}\left|\sum_{l=2}^{k} e_{l}^{\varepsilon_{j}}-\frac{k-1}{n_{\varepsilon_{j}}}\right|+C_{g} \frac{N_{\varepsilon_{j}}}{n_{\varepsilon_{j}}}\left(e_{1}^{\varepsilon_{j}}+\frac{1}{n_{\varepsilon_{j}}}\right) \\
& \leq C_{g} Z\left(\varepsilon_{j}\right)+C_{g} \frac{N_{\varepsilon_{j}}}{n_{\varepsilon_{j}}}\left(e_{1}^{\varepsilon_{j}}+\frac{1}{n_{\varepsilon_{j}}}\right)
\end{aligned}
$$

where $C_{g}$ is the Lipschitz constant of $g$ and

$$
Z(\varepsilon)=\frac{1}{n_{\varepsilon}} \sum_{k=2}^{n_{\varepsilon}^{1+\delta}}\left|\sum_{l=2}^{k} e_{l}^{\varepsilon}-\frac{(k-1)}{n_{\varepsilon}}\right| .
$$

In order to prove that $\lim _{j \rightarrow \infty} Z\left(\varepsilon_{j}\right)=0$, we compute the expectation of $Z(\varepsilon)$ :

$$
\mathbb{E}[Z(\varepsilon)]=\frac{1}{n_{\varepsilon}} \sum_{k=2}^{n_{\varepsilon}^{1+\delta}} \mathbb{E}\left[\left|\sum_{l=1}^{k} e_{l}^{\varepsilon}-\frac{k-1}{n_{\varepsilon}}\right|\right]=\frac{1}{n_{\varepsilon}^{2}} \sum_{k=2}^{n_{\varepsilon}^{1+\delta}} \mathbb{E}\left[\left|\sum_{l=2}^{k} n_{\varepsilon} e_{l}^{\varepsilon}-(k-1)\right|\right] .
$$


But, as the law $n_{\varepsilon} e_{l}^{\varepsilon}$ is the exponential law with parameter 1, we have

$$
\mathbb{E}\left[\left(\sum_{l=2}^{k} n_{\varepsilon} e_{l}^{\varepsilon}-(k-1)\right)^{4}\right]=6 k(k-1) .
$$

Thus, the quantity $\mathbb{E}[Z(\varepsilon)]$ is bounded from above by

$$
\frac{1}{n_{\varepsilon}^{2}} \sum_{k=2}^{n_{\varepsilon}^{1+\delta}} \mathbb{E}\left[\left(\sum_{l=2}^{k} n_{\varepsilon} e_{l}^{\varepsilon}-(k-1)\right)^{4}\right]^{1 / 4} \leq 2 \frac{1}{n_{\varepsilon}^{2}} \sum_{k=2}^{n_{\varepsilon}^{1+\delta}} \sqrt{k} \leq 2 n_{\varepsilon}^{3(1+\delta) / 2-2} \leq 2 n_{\varepsilon}^{-(1-3 \delta) / 2} .
$$

In particular, the series $\sum_{j \geq 1} \mathbb{E}\left[Z\left(\varepsilon_{j}\right)\right]$ converges and as $Z(\varepsilon)$ is non-negative, this implies the series $\sum_{j \geq 1} Z\left(\varepsilon_{j}\right)$ converges a.s. and thus $\mathbb{N}$-a.e. we have

$$
\lim _{j \rightarrow+\infty} Z\left(\varepsilon_{j}\right)=0 .
$$

The convergence of the Riemann's sums gives that $\mathbb{N}$-a.e.

$$
\frac{1}{n_{\varepsilon_{j}}} \sum_{k=2}^{N_{\varepsilon_{j}}} g\left(\frac{k}{n_{\varepsilon_{j}}}\right)=\frac{N_{\varepsilon_{j}}}{n_{\varepsilon_{j}}} \frac{1}{N_{\varepsilon_{j}}} \sum_{k=2}^{N_{\varepsilon_{j}}} g\left(\frac{N_{\varepsilon_{j}}}{n_{\varepsilon_{j}}} \frac{k}{N_{\varepsilon_{j}}}\right) \underset{j \rightarrow+\infty}{\longrightarrow} A_{\sigma} \int_{0}^{1} g\left(u A_{\sigma}\right) d u=\int_{0}^{A_{\sigma}} g(u) d u .
$$

Then we deduce (33) from (18), and this finishes the proof.

\subsection{Computation of the limit.}

Lemma 5.10. We assume $\left(h_{2}\right)$, that is $t \mapsto \phi(t, \omega, \mathcal{S})$ is continuous for all $\mathcal{S} \in \mathbb{S}$. We have, for the sequence $\left(\varepsilon_{j}, j \in \mathbb{N}^{*}\right)$ from Lemma [5.9, that $\mathbb{N}$-a.e.

$$
\lim _{j \rightarrow \infty} \prod_{k=2}^{N_{\varepsilon_{j}}} \mathrm{~N}\left[\mathrm{e}^{-\phi\left(A_{\left.S_{k}^{\varepsilon_{j}}, \omega, \cdot\right)}\right.} \mid \rho_{0}>\varepsilon_{j}\right]=\exp \left(-\int_{0}^{\tilde{\sigma}} d u \mathrm{~N}\left[1-\mathrm{e}^{-\phi(u, \omega, \cdot)}\right]\right) .
$$

Proof. For any sequence $\left(\varphi_{k}, k \in \mathbb{N}\right)$ of non-negative measurable function on $\mathbb{S}$, such that $\varphi_{k}(\mathcal{S})=0$ if $\left\langle\rho_{0}, 1\right\rangle \leq \eta$, we have for $\varepsilon \in(0, \eta)$,

$$
\prod_{k=2}^{N_{\varepsilon}} \mathrm{N}\left[\mathrm{e}^{-\varphi_{k}} \mid \rho_{0}>\varepsilon\right]=\prod_{k=2}^{N_{\varepsilon}}\left(1-\frac{\mathrm{N}\left[1-\mathrm{e}^{-\varphi_{k}}\right]}{\mathrm{N}\left[\rho_{0}>\varepsilon\right]}\right) \text {. }
$$

Recall (32), and notice that $\mathrm{N}\left[1-\mathrm{e}^{-\varphi_{k}}\right] \leq \mathrm{N}\left[\rho_{0} \geq \eta\right] \leq \mathrm{N}\left[\rho_{0}>\varepsilon\right]=n_{\varepsilon}$ and $\lim _{\varepsilon \downarrow 0} n_{\varepsilon}=+\infty$. Since $\log (1-x)=-x+h(x)$, with $|h(x)| \leq x^{2}$ for $x \in[0,1 / 2]$, we have for $\varepsilon$ small enough such that $\mathrm{N}\left[\rho_{0}>\eta\right] / n_{\varepsilon} \leq 1 / 2$,

$$
\begin{aligned}
\prod_{k=2}^{N_{\varepsilon}}\left(1-\frac{\mathrm{N}\left[1-\mathrm{e}^{-\varphi_{k}}\right]}{n_{\varepsilon}}\right) & =\exp \left(\sum_{k=2}^{N_{\varepsilon}} \ln \left(1-\frac{\mathrm{N}\left[1-\mathrm{e}^{-\varphi_{k}}\right]}{n_{\varepsilon}}\right)\right) \\
& =\exp \left(-\frac{1}{n_{\varepsilon}} \sum_{k=2}^{N_{\varepsilon}} \mathrm{N}\left[1-\mathrm{e}^{-\varphi_{k}}\right]\right) \exp \left(\sum_{k=2}^{N_{\varepsilon}} h\left(\mathrm{~N}\left[1-\mathrm{e}^{-\varphi_{k}}\right] / n_{\varepsilon}\right)\right)
\end{aligned}
$$

and $\sum_{k=2}^{N_{\varepsilon}} h\left(\mathrm{~N}\left[1-\mathrm{e}^{-\varphi_{k}}\right] / n_{\varepsilon}\right) \leq \mathrm{N}\left[\rho_{0}>\eta\right]^{2} N_{\varepsilon} / n_{\varepsilon}^{2}$. From the hypothesis on $\phi$, we can take $\varphi_{k}=\phi\left(A_{S_{k}^{\varepsilon_{j}}}, \omega, \cdot\right)$. Then, we deduce from Lemma 5.9 (i), that $\mathbb{N}$-a.e.

$$
\lim _{j \rightarrow \infty} \sum_{k=2}^{N_{\varepsilon_{j}}} h\left(\mathrm{~N}\left[1-\mathrm{e}^{-\phi\left(A_{\left.S_{k} \varepsilon_{j}, \omega, \cdot\right)}\right.}\right] / n_{\varepsilon_{j}}\right)=0 .
$$


Since $\left(h_{2}\right)$ is satisfied, we deduce that $t \mapsto \mathrm{N}\left[1-\mathrm{e}^{-\phi(t, \omega, \cdot)}\right]$ is continuous. We get from Lemma 5.9 (ii), that $\mathbb{N}$-a.e.

$$
\lim _{j \rightarrow \infty} \frac{1}{n_{\varepsilon_{j}}} \sum_{k=2}^{N_{\varepsilon_{j}}} \mathrm{~N}\left[1-\mathrm{e}^{-\phi\left(A_{\left.S_{k}^{\varepsilon_{j}}, \omega, \cdot\right)}\right.}\right]=\int_{0}^{\tilde{\sigma}} \mathrm{N}\left[1-\mathrm{e}^{-\phi(u, \omega, \cdot)}\right] d u .
$$

This finishes the proof of the Lemma.

5.7. Proof of Theorem [5.2, Let $Z \in \tilde{\mathcal{F}}_{\infty}$ non-negative such that $\mathbb{N}[Z]<\infty$. Let $\phi$ satisfying hypothesis of Theorem [5.2. $\left(h_{1}\right)$ and $\left(h_{2}\right)$. We have

$$
\begin{aligned}
\mathbb{N}\left[Z \exp \left(-\sum_{i \in I} \phi\left(A_{\alpha_{i}}, \omega, \mathcal{S}^{i}\right)\right)\right. & =\lim _{j \rightarrow \infty} \mathbb{N}\left[Z \exp \left(-\sum_{k=2}^{N_{\varepsilon_{j}}} \phi\left(A_{\left.S_{k}^{\varepsilon_{j}}, \omega, \mathcal{S}^{k, \varepsilon_{j}}\right)}\right)\right]\right. \\
& =\lim _{j \rightarrow \infty} \mathbb{N}\left[Z \prod_{k=2}^{N_{\varepsilon_{j}}} \mathrm{~N}\left[\mathrm{e}^{-\phi\left(A_{\left.S_{k}^{\varepsilon_{j}}, \omega, \cdot\right)}\right.} \mid \rho_{0}>\varepsilon_{j}\right]\right] \\
& =\mathbb{N}\left[Z \exp \left(-\int_{0}^{\tilde{\sigma}} \mathrm{N}\left[1-\mathrm{e}^{-\phi(u, \omega, \cdot)}\right] d u\right)\right],
\end{aligned}
$$

where we used Lemma 5.4 and dominated convergence for the first equality, Lemma 5.7 for the second equality, Lemma 5.10 and dominated convergence for the last equality. By monotone class Theorem (resp. monotonicity), we can remove hypothesis $\left(h_{2}\right)$ (resp. $\left(h_{1}\right)$ ). To ends the proof, it suffices to remark that $\exp \left(-\int_{0}^{\tilde{\sigma}} \mathrm{N}\left[1-\mathrm{e}^{-\phi(u, \omega, \cdot)}\right] d u\right)$ is $\tilde{\mathcal{F}}_{\infty}$-measurable and so this is $\mathbb{N}$-a.e. equal to the conditional expectation (i.e. the left hand side term of (24)).

\section{LAW OF THE PRUNED EXPLORATION PROCESS}

Recall notations of Section 3 and definition (14). We still fix $\theta>0$ and write $m$ for $m^{(\theta)}$. Notice that $\psi^{(\theta)}=\psi(\theta+\cdot)-\psi(\theta)$, defined by (17) is the Laplace exponent of a Lévy process, with Lévy measure satisfying (1). The exploration process, $\rho^{(\theta)}$, of this Lévy process is thus well defined.

The aim of this section is to prove the following Theorem.

Theorem 6.1. For every finite measure $\mu$, the law of the pruned process $\tilde{\rho}$ under $\mathbb{P}_{\mu, 0}$ is the law of the exploration process $\rho^{(\theta)}$ associated to a Lévy process with Laplace exponent $\psi^{(\theta)}$ under $\mathbb{P}_{\mu}$.

The next Corollary is a direct consequence of this Theorem.

Corollary 6.2. The excursion measure of $\tilde{\rho}$ outside 0 is equal to the excursion measure of $\rho^{(\theta)}$ outside 0 .

6.1. A martingale problem for $\tilde{\rho}$. In this section, we shall compute the law of the total mass process $\left(\left\langle\tilde{\rho}_{t \wedge \tilde{\sigma}}, 1\right\rangle, t \geq 0\right)$ under $\mathbb{P}_{\mu}=\mathbb{P}_{\mu, 0}$, using martingale problem characterization. We will first show how a martingale problem for $\rho$ can be translated into a martingale problem for $\tilde{\rho}$. (In a forthcoming paper, we shall compute the infinitesimal generator of $\rho$ for exponential functionals.) Unfortunately, we were not able to use standard techniques of random time change, as developed in Chapter 6 of 12 and used for Poisson snake in 11, mainly because $t^{-1}\left[\mathbb{E}_{\mu}\left[f\left(\rho_{t}\right) \mathbf{1}_{\left\{m_{t}=0\right\}}\right]-f(\mu)\right]$ does not have a limit as $t$ goes down to 0 , even for exponential functionals. 
Let $F, K \in \mathcal{B}\left(\mathcal{M}_{f}\left(\mathbb{R}_{+}\right)\right)$bounded such that, for any $\mu \in \mathcal{M}_{f}\left(R_{+}\right), \mathbb{E}_{\mu}\left[\int_{0}^{\sigma}\left|K\left(\rho_{s}\right)\right| d s\right]<$ $\infty$ and $M_{t}=F\left(\rho_{t \wedge \sigma}\right)-\int_{0}^{t \wedge \sigma} K\left(\rho_{s}\right)$, for $t \geq 0$, define an $\mathcal{F}$-martingale. In particular, notice that $\mathbb{E}_{\mu}\left[\sup _{t>0}\left|M_{t}\right|\right]<\infty$. Thus, we can define for $t \geq 0$,

$$
N_{t}=\mathbb{E}_{\mu}^{*}\left[M_{C_{t}} \mid \tilde{\mathcal{F}}_{t}\right] .
$$

Proposition 6.3. The process $N=\left(N_{t}, t \geq 0\right)$ is an $\tilde{\mathcal{F}}$-martingale. And we have for all $\mu \in \mathcal{M}_{f}\left(\mathbb{R}_{+}\right), \mathbb{P}_{\mu^{-}}$a.s.

$$
\int_{0}^{\tilde{\sigma}} d u \int_{(0, \infty)}\left(1-\mathrm{e}^{-\theta \ell}\right) \pi(d \ell)\left|F\left(\left[\tilde{\rho}_{u}, \ell \delta_{0}\right]\right)-F\left(\tilde{\rho}_{u}\right)\right|<\infty
$$

and the representation formula for $N_{t}$ :

$$
N_{t}=F\left(\tilde{\rho}_{t \wedge \tilde{\sigma}}\right)-\int_{0}^{t \wedge \tilde{\sigma}} d u\left(K\left(\tilde{\rho}_{u}\right)+\int_{(0, \infty)}\left(1-\mathrm{e}^{-\theta \ell}\right) \pi(d \ell)\left(F\left(\left[\tilde{\rho}_{u}, \ell \delta_{0}\right]\right)-F\left(\tilde{\rho}_{u}\right)\right)\right) .
$$

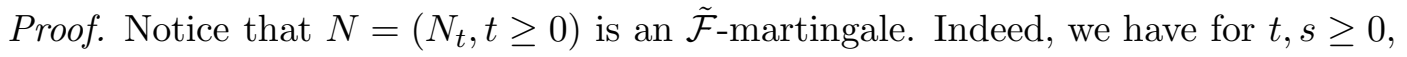

$$
\begin{aligned}
\mathbb{E}_{\mu}\left[N_{t+s} \mid \tilde{\mathcal{F}}_{t}\right] & =\mathbb{E}_{\mu}\left[\mathbb{E}_{\mu}\left[M_{C_{t+s}} \mid \tilde{\mathcal{F}}_{t+s}\right] \mid \tilde{\mathcal{F}}_{t}\right] \\
& =\mathbb{E}_{\mu}\left[M_{C_{t+s}} \mid \tilde{\mathcal{F}}_{t}\right] \\
& =\mathbb{E}_{\mu}\left[\mathbb{E}_{\mu}\left[M_{C_{t+s}} \mid \mathcal{F}_{C_{t}}\right] \mid \tilde{\mathcal{F}}_{t}\right] \\
& =\mathbb{E}_{\mu}\left[M_{C_{t}} \mid \tilde{\mathcal{F}}_{t}\right],
\end{aligned}
$$

where we used the stopping time Theorem for the last equality. To compute $\mathbb{E}_{\mu}\left[M_{C_{t}} \mid \tilde{\mathcal{F}}_{t}\right]$, we write $M_{C_{t}}=N_{t}^{\prime}-M_{C_{t}}^{\prime}$, where for $u \geq 0$,

$$
M_{u}^{\prime}=\int_{0}^{u \wedge \sigma} K\left(\rho_{s}\right) \mathbf{1}_{\left\{m_{s} \neq 0\right\}} d s .
$$

Recall that $C_{0}=0 \mathbb{P}_{\mu}$-a.s. by Corollary 4.2, In particular, we get

$$
\begin{aligned}
N_{t}^{\prime} & =F\left(\rho_{C_{t} \wedge \sigma}\right)-\int_{0}^{C_{t} \wedge \sigma} K\left(\rho_{s}\right) \mathbf{1}_{\left\{m_{s}=0\right\}} d s \\
& =F\left(\tilde{\rho}_{t \wedge \tilde{\sigma}}\right)-\int_{0}^{C_{t} \wedge \sigma} K\left(\rho_{s}\right) d A_{s} \\
& =F\left(\tilde{\rho}_{t \wedge \tilde{\sigma}}\right)-\int_{0}^{t \wedge \tilde{\sigma}} K\left(\tilde{\rho}_{u}\right) d u,
\end{aligned}
$$

where we used the time change $u=A_{s}$ for the last equality. In particular, as $\tilde{\sigma}$ is an $\tilde{\mathcal{F}}_{\text {- }}$ stopping time, we get that the process $\left(N_{t}^{\prime}, t \geq 0\right)$ is $\tilde{\mathcal{F}}$-adapted. Since $N_{t}=N_{t}^{\prime}-\mathbb{E}_{\mu}\left[M_{C_{t}}^{\prime} \mid \tilde{\mathcal{F}}_{t}\right]$, we are left with the computation of $\mathbb{E}_{\mu}\left[M_{C_{t}}^{\prime} \mid \tilde{\mathcal{F}}_{t}\right]$.

In Section [5] the arguments are given under the excursion measure, but they can readily be extended under $\mathbb{P}_{\mu}$ or $\mathbb{P}_{\mu, 0}^{*}$. In particular, the result of Corollary 5.3 holds also under $\mathbb{P}_{\mu}$ or $\mathbb{P}_{\mu, 0}^{*}$. We keep the notations of Section [5. We consider $\left(\rho^{i}, m^{i}\right), i \in I$ the excursions of the process $(\rho, m)$ outside $\left\{s, m_{s}=0\right\}$ before $\sigma$ and let $\left(\alpha_{i}, \beta_{i}\right), i \in I$ be the corresponding interval excursions. In particular we can write

$$
\int_{0}^{C_{t} \wedge \sigma}\left|K\left(\rho_{s}\right)\right| \mathbf{1}_{\left\{m_{s} \neq 0\right\}} d s=\sum_{i \in I} \Phi\left(A_{\alpha_{i}}, \rho_{\alpha_{i}-}, \rho_{i}\right),
$$


with

$$
\Phi(u, \mu, \rho)=\mathbf{1}_{\{u<t\}} \int_{0}^{\sigma(\rho)}\left|K\left(\left[\mu, \rho_{s}\right]\right)\right| d s,
$$

where $\sigma(\rho)=\inf \left\{v>0 ; \rho_{v}=0\right\}$. We deduce from Corollary 5.3 that $\mathbb{P}_{\mu}$-a.s.

$$
\mathbb{E}_{\mu}\left[\int_{0}^{C_{t} \wedge \sigma}\left|K\left(\rho_{s}\right)\right| \mathbf{1}_{\left\{m_{s} \neq 0\right\}} d s \mid \tilde{\mathcal{F}}_{\infty}\right]=\int_{0}^{\tilde{\sigma}} \mathbf{1}_{\{u<t\}} \hat{K}\left(\tilde{\rho}_{u}\right) d u
$$

with, $\hat{K}$ defined for $\nu \in \mathcal{M}_{f}\left(\mathbb{R}_{+}\right)$by

$$
\hat{K}(\nu)=\int_{(0, \infty)}\left(1-\mathrm{e}^{-\theta \ell}\right) \pi(d \ell) \mathbb{E}_{\ell}\left[\int_{0}^{\sigma}\left|K\left(\left[\nu, \rho_{s}\right]\right)\right| d s\right] .
$$

Since $\mathbb{E}_{\mu}\left[\int_{0}^{\sigma}\left|K\left(\rho_{s}\right)\right| d s\right]$ is finite, we deduce that $\mathbb{P}_{\mu^{-}}$a.s. $d u$-a.e. $\mathbf{1}_{\{u<\tilde{\sigma}\}} \hat{K}\left(\tilde{\rho}_{u}\right)$ is finite.

We define $\tilde{K} \in \mathcal{B}\left(\mathcal{M}_{f}\left(\mathbb{R}_{+}\right)\right)$for $\nu \in \mathcal{M}_{f}\left(\mathbb{R}_{+}\right)$by

$$
\tilde{K}(\nu)=\int_{(0, \infty)}\left(1-\mathrm{e}^{-\theta \ell}\right) \pi(d \ell) \mathbb{E}_{\ell}\left[\int_{0}^{\sigma} K\left(\left[\nu, \rho_{s}\right]\right) d s\right]
$$

if $\hat{K}(\nu)<\infty$, or by $\tilde{K}(\nu)=0$ if $\hat{K}(\nu)=+\infty$. In particular, we have $|\tilde{K}(\nu)| \leq \hat{K}(\nu)$ and $\mathbb{P}_{\mu}$-a.s. $\int_{0}^{\tilde{\sigma}}\left|\tilde{K}\left(\tilde{\rho}_{u}\right)\right| d u$ is finite. Using Corollary 5.3 once again (see (35)), we get that $\mathbb{P}_{\mu}$-a.s.,

$$
\mathbb{E}_{\mu}\left[M_{C_{t}}^{\prime} \mid \tilde{\mathcal{F}}_{\infty}\right]=\mathbb{E}_{\mu}\left[\int_{0}^{C_{t} \wedge \sigma} K\left(\rho_{s}\right) \mathbf{1}_{\left\{m_{s} \neq 0\right\}} d s \mid \tilde{\mathcal{F}}_{\infty}\right]=\int_{0}^{t \wedge \tilde{\sigma}} \tilde{K}\left(\tilde{\rho}_{u}\right) d u
$$

To rewrite $\tilde{K}$, we notice that, for $\nu$ with compact support, $\mathbb{E}_{\ell}\left[\int_{0}^{\sigma} K\left(\left[\nu, \rho_{s}\right]\right) d s\right]$ is equal to $\mathbb{E}_{\left[\nu, \ell \delta_{0}\right]}\left[\int_{0}^{\tau_{\ell}} K\left(\rho_{s}\right) d s\right]$, where $\tau_{\ell}=\inf \left\{s ;-I_{s} \geq \ell\right\}$ is an $\mathcal{F}$-stopping time. Notice that $\mathbb{P}_{\left[\nu, \ell \delta_{0}\right]^{-a . s .}} \tau_{\ell} \leq \sigma$ and $\rho_{\tau_{\ell}}=\nu$. We deduce from the stopping time Theorem that

$$
\mathbb{E}_{\left[\nu, \ell \delta_{0}\right]}\left[\int_{0}^{\tau_{\ell}} K\left(\rho_{s}\right) d s\right]=\mathbb{E}_{\left[\nu, \ell \delta_{0}\right]}\left[-M_{\tau_{\ell}}+F\left(\rho_{\tau_{\ell}}\right)\right]=-F\left(\left[\nu, \ell \delta_{0}\right]\right)+F(\nu) .
$$

Therefore, we get from (36) and (37)

$$
\mathbb{E}_{\mu}\left[M_{C_{t}}^{\prime} \mid \tilde{\mathcal{F}}_{\infty}\right]=-\int_{0}^{t \wedge \tilde{\sigma}} \int_{(0, \infty)}\left(1-\mathrm{e}^{-\theta \ell}\right) \pi(d \ell)\left(F\left(\left[\tilde{\rho}_{u}, \ell \delta_{0}\right]\right)-F\left(\tilde{\rho}_{u}\right)\right) d u .
$$

Eventually, as $N_{t}=N_{t}^{\prime}-\mathbb{E}_{\mu}\left[M_{C_{t}}^{\prime} \mid \tilde{\mathcal{F}}_{\infty}\right]$, this gives (34).

To conclude, notice that from (38), the definition of $\hat{K}$ and (35), we have

$$
\begin{aligned}
\int_{0}^{\tilde{\sigma}} \int_{(0, \infty)}\left(1-\mathrm{e}^{-\theta \ell}\right) \pi(d \ell) \mid & F\left(\left[\tilde{\rho}_{u}, \ell \delta_{0}\right]\right)-F\left(\tilde{\rho}_{u}\right) \mid d u \\
& \leq \int_{0}^{\tilde{\sigma}} \int_{(0, \infty)}\left(1-\mathrm{e}^{-\theta \ell}\right) \pi(d \ell) \mathbb{E}_{\left[\tilde{\rho}_{u}, \ell \delta_{0}\right]}\left[\int_{0}^{\tau_{\ell}}\left|K\left(\rho_{s}\right)\right| d s\right] d u \\
& =\int_{0}^{\tilde{\sigma}} \hat{K}\left(\tilde{\rho}_{u}\right) d u \\
& =\mathbb{E}_{\mu}\left[\int_{0}^{\sigma}\left|K\left(\rho_{s}\right)\right| \mathbf{1}_{\left\{m_{s} \neq 0\right\}} d s \mid \tilde{\mathcal{F}}_{\infty}\right],
\end{aligned}
$$


which is finite $\mathbb{P}_{\mu}$-a.s. since $\mathbb{E}_{\mu}\left[\int_{0}^{\sigma}\left|K\left(\rho_{s}\right)\right| d s\right]<\infty$.

Corollary 6.4. Let $\mu \in \mathcal{M}_{f}\left(\mathbb{R}_{+}\right)$. The law of the total mass process $\left(\left\langle\tilde{\rho}_{t}, 1\right\rangle, t \geq 0\right)$ under $\mathbb{P}_{\mu, 0}^{*}$ is the law of the total mass process of $\rho^{(\theta)}$ under $\mathbb{P}_{\mu}^{*}$.

Proof. Let $X=\left(X_{t}, t \geq 0\right)$ be under $\mathrm{P}_{x}^{*}$, a Lévy process with Laplace transform $\psi$ started at $x>0$ and stopped when it reached 0 . Under $\mathbb{P}_{\mu}$, the total mass process $\left(\left\langle\rho_{t \wedge \sigma}, 1\right\rangle, t \geq 0\right)$ is distributed as $X$ under $\mathrm{P}_{\langle\mu, 1\rangle}^{*}$. Let $c>0$. From Lévy processes theory, we know that the process $\mathrm{e}^{-c X_{t}}-\psi(c) \int_{0}^{t} \mathrm{e}^{-c X_{s}} d s$, for $t \geq 0$ is a martingale. We deduce from the stopping time Theorem that $M=\left(M_{t}, t \geq 0\right)$ is an $\mathcal{F}$-martingale under $\mathbb{P}_{\mu}$, where $M_{t}=F\left(\rho_{t \wedge \sigma}\right)-$ $\int_{0}^{t \wedge \sigma} K\left(\rho_{s}\right) d s$, with $F, K \in \mathcal{B}\left(\mathcal{M}_{f}\left(\mathbb{R}_{+}\right)\right)$defined by $F(\nu)=\mathrm{e}^{-c\langle\nu, 1\rangle}$ for $\nu \in \mathcal{M}_{f}\left(\mathbb{R}_{+}\right)$and $K=\psi(c) F$. Notice $K \geq 0$. We have by dominated convergence and monotone convergence.

$$
\mathrm{e}^{-c\langle\mu, 1\rangle}=\lim _{t \rightarrow \infty} \mathbb{E}_{\mu}\left[M_{t}\right]=\mathbb{E}_{\mu}\left[\mathrm{e}^{-c\left\langle\rho_{\sigma}, 1\right\rangle}\right]-\psi(c) \mathbb{E}_{\mu}\left[\int_{0}^{\sigma} \mathrm{e}^{-c\left\langle\rho_{s}, 1\right\rangle} d s\right]
$$

This implies that, for any $\mu \in \mathcal{M}_{f}\left(R_{+}\right), \mathbb{E}_{\mu}\left[\int_{0}^{\sigma}\left|K\left(\rho_{s}\right)\right| d s\right]$ is finite. For $\nu \in \mathcal{M}_{f}\left(\mathbb{R}_{+}\right)$with compact support, we have

$$
\begin{aligned}
\int_{(0, \infty)}\left(1-\mathrm{e}^{-\theta \ell}\right) \pi(d \ell) \mid F\left(\left[\nu, \ell \delta_{0}\right]\right)-F & (\nu) \mid \\
& =\int_{(0, \infty)}\left(1-\mathrm{e}^{-\theta \ell}\right) \pi(d \ell)\left(\mathrm{e}^{-c\langle\nu, 1\rangle}-\mathrm{e}^{-c\langle\nu, 1\rangle-c \ell}\right) \\
& =\mathrm{e}^{-c\langle\nu, 1\rangle} \int_{(0, \infty)}\left(1-\mathrm{e}^{-\theta \ell}\right)\left(1-\mathrm{e}^{-c \ell}\right) \pi(d \ell) \\
& =\mathrm{e}^{-c\langle\nu, 1\rangle}\left(\psi(c)-\psi^{(\theta)}(c)\right) .
\end{aligned}
$$

In particular, we have

$$
\int_{(0, \infty)}\left(1-\mathrm{e}^{-\theta \ell}\right) \pi(d \ell)\left[F\left(\left[\tilde{\rho}_{u}, \ell \delta_{0}\right]\right)-F\left(\tilde{\rho}_{u}\right)\right]=\mathrm{e}^{-c\left\langle\tilde{\rho}_{u}, 1\right\rangle}\left(\psi^{(\theta)}(c)-\psi(c)\right) .
$$

From Proposition 6.3. we get that $N=\left(N_{t}, t \geq 0\right)$, with for $t \geq 0$,

$$
N_{t}=\mathrm{e}^{-c\left\langle\tilde{\rho}_{t \wedge \tilde{\sigma}}, 1\right\rangle}-\psi^{(\theta)}(c) \int_{0}^{t \wedge \tilde{\sigma}} \mathrm{e}^{-c\left\langle\tilde{\rho}_{s}, 1\right\rangle} d s
$$

is under $\mathbb{P}_{\mu}$ an $\tilde{\mathcal{F}}$-martingale.

Notice that $\tilde{\sigma}=\inf \left\{s \geq 0 ;\left\langle\tilde{\rho}_{s}, 1\right\rangle=0\right\}$. Let $X^{(\theta)}=\left(X_{t}^{(\theta)}, t \geq 0\right)$ be under $\mathrm{P}_{x}^{*}$, a Lévy process with Laplace transform $\psi^{(\theta)}$ started at $x>0$ and stopped when it reached 0 . The two non-negative càd-làg processes $\left(\left\langle\tilde{\rho}_{t \wedge \tilde{\sigma}}, 1\right\rangle, t \geq 0\right)$ and $X^{(\theta)}$ solves the martingale problem: for any $c \geq 0$, the process defined for $t \geq 0$ by

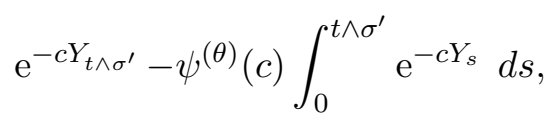

where $\sigma^{\prime}=\inf \left\{s \geq 0 ; Y_{s} \leq 0\right\}$, is a martingale. From Corollary 4.4.4 in [12, we deduce that those two processes have the same distribution. To finish the proof, notice that the total mass process of $\rho^{(\theta)}$ under $\mathbb{P}_{\mu}^{*}$ is distributed as $X^{(\theta)}$ under $\mathrm{P}_{\langle\mu, 1\rangle}^{*}$. 
6.2. Identification of the law of $\tilde{\rho}$. To begin with, let us mention some useful properties of the process $\tilde{\rho}$.

Lemma 6.5. We have the following properties for the process $\tilde{\rho}$.

(i) $\tilde{\rho}$ is a càd-làg Markov process.

(ii) The sojourn time at 0 of $\tilde{\rho}$ is 0 .

(iii) 0 is recurrent for $\tilde{\rho}$.

Proof. (i) This is a direct consequence of the strong Markov property of the process $(\rho, m)$.

(ii) We have for $r>0$, with the change of variable $t=A_{s}$, a.s.

$$
\int_{0}^{r} \mathbf{1}_{\left\{\tilde{\rho}_{t}=0\right\}} d t=\int_{0}^{r} \mathbf{1}_{\left\{\rho_{C_{t}}=0\right\}} d t=\int_{0}^{C_{r}} \mathbf{1}_{\left\{\rho_{s}=0\right\}} d A_{s}=\int_{0}^{C_{r}} \mathbf{1}_{\left\{\rho_{s}=0\right\}} d s=0,
$$

as the sojourn time of $\rho$ at 0 is 0 a.s.

(iii) Since $\tilde{\sigma}=A_{\sigma}$ and $\sigma<+\infty$ a.s., we deduce that 0 is recurrent for $\tilde{\rho}$ a.s.

Since the processes $\tilde{\rho}$ and $\rho^{(\theta)}$ are both Markov processes, to show that they have the same law, it is enough to show that they have the same one-dimensional marginals. We first prove that result under the excursion measure.

Proposition 6.6. For every $\lambda>0$ and every non-negative bounded measurable function $f$,

$$
\mathbb{N}\left[\int_{0}^{\tilde{\sigma}} \mathrm{e}^{-\lambda t-\left\langle\tilde{\rho}_{t}, f\right\rangle} d t\right]=\mathbb{N}\left[\int_{0}^{\sigma^{(\theta)}} \mathrm{e}^{-\lambda t-\left\langle\rho_{t}^{(\theta)}, f\right\rangle} d t\right]
$$

Proof. On one hand, we compute, using the definition of the pruned process $\tilde{\rho}$,

$$
\mathbb{N}\left[\int_{0}^{\tilde{\sigma}} \mathrm{e}^{-\lambda t-\left\langle\tilde{\rho}_{t}, f\right\rangle} d t\right]=\mathbb{N}\left[\int_{0}^{A_{\sigma}} \mathrm{e}^{-\lambda t-\left\langle\rho_{C_{t}}, f\right\rangle} d t\right] .
$$

We now make the change of variable $t=A_{u}$ to get

$$
\begin{aligned}
\mathbb{N}\left[\int_{0}^{\tilde{\sigma}} \mathrm{e}^{-\lambda t-\left\langle\tilde{\rho}_{t}, f\right\rangle} d t\right] & =\mathbb{N}\left[\int_{0}^{\sigma} \mathrm{e}^{-\lambda A_{u}} \mathrm{e}^{-\left\langle\rho_{u}, f\right\rangle} d A_{u}\right] \\
& =\mathbb{N}\left[\int_{0}^{\sigma} \mathrm{e}^{-\lambda A_{u}} \mathrm{e}^{-\left\langle\rho_{u}, f\right\rangle} \mathbf{1}_{\left\{m_{u}=0\right\}} d u\right]
\end{aligned}
$$

By a time reversibility argument, see Lemma 3.6, we obtain

$$
\begin{aligned}
& \mathbb{N}\left[\int_{0}^{\tilde{\sigma}} \mathrm{e}^{-\lambda t-\left\langle\tilde{\rho}_{t}, f\right\rangle} d t\right]=\mathbb{N}\left[\int_{0}^{\sigma} \mathbf{1}_{\left\{m_{u}=0\right\}} \mathrm{e}^{-\left\langle\eta_{u}, f\right\rangle} \mathrm{e}^{-\lambda\left(A_{\sigma}-A_{u}\right)} d u\right] \\
& =\mathbb{N}\left[\int_{0}^{\sigma} \mathbf{1}_{\left\{m_{u}=0\right\}} \mathrm{e}^{-\left\langle\eta_{u}, f\right\rangle} \mathbb{E}_{\rho_{u}, 0}^{*}\left[\mathrm{e}^{-\lambda A_{\sigma}}\right] d u\right] \\
& =\mathbb{N}\left[\int_{0}^{\sigma} \mathbf{1}_{\left\{m_{u}=0\right\}} \mathrm{e}^{-\left\langle\eta_{u}, f\right\rangle} \mathrm{e}^{-\left\langle\rho_{u}, 1\right\rangle \psi^{(\theta)}-1}(\lambda) d u\right]
\end{aligned}
$$

where we applied Lemma 4.1 (i) for the last equality. Now, by definition of $m$, we have by conditioning,

$$
\mathbb{N}\left[\int_{0}^{\tilde{\sigma}} \mathrm{e}^{-\lambda t-\left\langle\tilde{\rho}_{t}, f\right\rangle} d t\right]=\mathbb{N}\left[\int_{0}^{\sigma} \mathrm{e}^{-\theta\left\langle\kappa_{u}, 1\right\rangle} \mathrm{e}^{-\left\langle\eta_{u}, f\right\rangle} \mathrm{e}^{-\left\langle\rho_{u}, 1\right\rangle \psi^{(\theta)-1}(\lambda)} d u\right] .
$$


Now, the Poisson decomposition of Proposition 2.6 and standard computations lead to

$$
\begin{aligned}
\mathbb{N} & {\left[\int_{0}^{\tilde{\sigma}} \mathrm{e}^{-\lambda t-\left\langle\tilde{\rho}_{t}, f\right\rangle} d t\right] } \\
& \left.=\int_{0}^{+\infty} d a \mathrm{e}^{-\alpha_{0} a} \exp \left\{-\int_{0}^{a} d x \int_{0}^{1} d u \int_{(0,+\infty)} \ell \pi(d \ell)\left[1-\mathrm{e}^{-\ell\left(\theta+(1-u) f(x)+u \psi^{(\theta)}-1\right.}(\lambda)\right)\right]\right\} \\
& =\int_{0}^{+\infty} d a \exp \left\{-\int_{0}^{a} d x \int_{0}^{1} d u \psi^{\prime}\left(\theta+(1-u) f(x)+u \psi^{(\theta)^{-1}}(\lambda)\right)\right\} \\
& =\int_{0}^{+\infty} d a \exp \left\{-\int_{0}^{a} d x \frac{\lambda-\psi^{(\theta)}(f(x))}{\psi^{(\theta)^{-1}}(\lambda)-f(x)}\right\} .
\end{aligned}
$$

On the other hand, the formula of Proposition [2.7 the Poisson representation of Proposition [2.6] and the same computations as before yields

$$
\begin{aligned}
\mathbb{N} & {\left[\int_{0}^{\sigma^{(\theta)}} \mathrm{e}^{-\lambda t-\left\langle\rho_{t}^{(\theta)}, f\right\rangle} d t\right] } \\
& =\int \mathbb{M}(d \mu d \nu) \mathrm{e}^{-\langle\mu, f\rangle} \mathrm{e}^{-\psi^{(\theta)}(\lambda)\langle\nu, 1\rangle} \\
& =\int_{0}^{+\infty} d a \mathrm{e}^{-\alpha_{0} a} \exp \left\{-\int_{0}^{a} d x \int_{1} d u \int_{(0,+\infty)} \ell \pi^{(\theta)}(d \ell)\left[1-\mathrm{e}^{-\ell\left(u f(x)+\psi^{(\theta)-1}(\lambda)(1-u)\right)}\right]\right\} \\
& =\int_{0}^{+\infty} d a \exp \left\{-\int_{0}^{a} d x \frac{\lambda-\psi^{(\theta)}(f(x))}{\psi^{(\theta)^{-1}}(\lambda)-f(x)}\right\} .
\end{aligned}
$$

As the two quantities are equal, the proof is over.

Now, we prove the same result under $\mathbb{P}_{\mu, 0}^{*}$, that is:

Proposition 6.7. For every $\lambda>0, f \in \mathcal{B}_{+}\left(\mathbb{R}_{+}\right)$bounded and every finite measure $\mu$,

$$
\mathbb{E}_{\mu, 0}^{*}\left[\int_{0}^{\tilde{\sigma}} \mathrm{e}^{-\lambda t-\left\langle\tilde{\rho}_{t}, f\right\rangle} d t\right]=\mathbb{E}_{\mu}^{*}\left[\int_{0}^{\sigma^{(\theta)}} \mathrm{e}^{-\lambda t-\left\langle\rho_{t}^{(\theta)}, f\right\rangle} d t\right] .
$$

Proof. From the Poisson representation, see Lemma 3.3. and using notations of this Lemma and of (19) we have

$$
\begin{aligned}
\mathbb{E}_{\mu, 0}^{*}\left[\int_{0}^{\tilde{\sigma}} \mathrm{e}^{-\lambda t-\left\langle\tilde{\rho}_{t}, f\right\rangle} d t\right] & =\mathbb{E}_{\mu, 0}^{*}\left[\int_{0}^{\sigma} \mathrm{e}^{-\lambda A_{u}-\left\langle\rho_{u}, f\right\rangle} d A_{u}\right] \\
& =\mathbb{E}_{\mu, 0}^{*}\left[\sum_{i \in J} \mathrm{e}^{-\lambda A_{\alpha_{i}}-\left\langle k_{-I \alpha_{i}}, f\right\rangle} \int_{0}^{\sigma_{i}} \mathrm{e}^{-\left\langle\rho_{s}^{i}, f_{-I_{\alpha_{i}}}\right\rangle-\lambda A_{s}^{i}} d A_{s}^{i}\right]
\end{aligned}
$$

where the function $f_{r}$ is defined by $f_{r}(x)=f\left(H_{r}^{(\mu)}+x\right)$ and $H_{r}^{(\mu)}=H\left(k_{r} \mu\right)$ is the maximal element of the closed support of $k_{r} \mu$ (see (5)). We recall that $-I$ is the local time at 0 of the reflected process $X-I$, and that $\tau_{r}=\inf \left\{s ;-I_{s}>r\right\}$ is the right continuous inverse of $-I$. 
From excursion formula, and using the time change $-I_{s}=r$ (or equivalently $\tau_{r}=s$ ), we get

$$
\begin{aligned}
\mathbb{E}_{\mu, 0}^{*}\left[\int_{0}^{\tilde{\sigma}} \mathrm{e}^{-\lambda t-\left\langle\tilde{\rho}_{t}, f\right\rangle} d t\right] & =\mathbb{E}_{\mu, 0}^{*}\left[\int_{0}^{\tau\langle\mu, 1\rangle} d\left(-I_{s}\right) \mathrm{e}^{-\left\langle k_{-I_{s}} \mu, f\right\rangle-\lambda A_{s}} G\left(-I_{s}\right)\right] \\
& =\mathbb{E}_{\mu, 0}^{*}\left[\int_{0}^{\langle\mu, 1\rangle} d r \mathrm{e}^{-\left\langle k_{r} \mu, f\right\rangle-\lambda A_{\tau_{r}}} G(r)\right]
\end{aligned}
$$

where the function $G(r)$ is given by

$$
G(r)=\mathbb{N}\left[\int_{0}^{\sigma} \mathrm{e}^{-\left\langle\rho_{s}, f_{r}\right\rangle-\lambda A_{s}} d A_{s}\right]=\mathbb{N}\left[\int_{0}^{\tilde{\sigma}} \mathrm{e}^{-\lambda t-\left\langle\tilde{\rho}_{t}, f_{r}\right\rangle} d t\right] .
$$

The same kind of computation gives

$$
\mathbb{E}_{\mu}^{*}\left[\int_{0}^{\sigma^{(\theta)}} \mathrm{e}^{-\lambda t-\left\langle\rho_{t}^{(\theta)}, f\right\rangle} d t\right]=\mathbb{E}\left[\int_{0}^{\langle\mu, 1\rangle} d r \mathrm{e}^{-\left\langle k_{r} \mu, f\right\rangle-\lambda \tau_{r}^{(\theta)}} G^{(\theta)}(r)\right]
$$

where the function $G^{(\theta)}$ is defined by

$$
G^{(\theta)}(r)=\mathbb{N}\left[\int_{0}^{\sigma^{(\theta)}} \mathrm{e}^{-\lambda s-\left\langle\rho_{s}^{(\theta)}, f_{r}\right\rangle} d s\right]
$$

and $\tau^{(\theta)}$ is the right-continuous inverse of the infimum process $-I^{(\theta)}$ of the Lévy process with Laplace exponent $\psi^{(\theta)}$.

Proposition 6.6 says that the functions $G$ and $G^{(\theta)}$ are equal. Moreover, as the total mass processes have the same law (see Corollary 6.4), we know that the proposition is true for $f$ constant. And, for $f$ constant, the functions $G$ and $G^{(\theta)}$ are also constant. Therefore, we have for $f$ constant equal to $c \geq 0$,

$$
\mathbb{E}_{\mu, 0}^{*}\left[\int_{0}^{\langle\mu, 1\rangle} d r \mathrm{e}^{-c(\langle\mu, 1\rangle-r)} \mathrm{e}^{-\lambda A_{\tau_{r}}}\right]=\mathbb{E}\left[\int_{0}^{\langle\mu, 1\rangle} d r \mathrm{e}^{-c(\langle\mu, 1\rangle-r)} \mathrm{e}^{-\lambda \tau_{r}^{(\theta)}}\right] .
$$

As this is true for any $c \geq 0$, uniqueness of the Laplace transform gives the equality

$$
\mathbb{E}_{\mu, 0}^{*}\left[\mathrm{e}^{-\lambda A_{\tau_{r}}}\right]=\mathbb{E}\left[\mathrm{e}^{-\lambda \tau_{r}^{(\theta)}}\right] \quad d r \text { - a.e. }
$$

In fact this equality holds for every $r$ by right-continuity.

Eventually as $G=G^{(\theta)}$, we have thanks to (39) and (40), that, for every bounded nonnegative measurable function $f$,

$$
\int_{0}^{\langle\mu, 1\rangle} d r \mathrm{e}^{-\left\langle k_{r} \mu, f\right\rangle} \mathbb{E}_{\mu, 0}^{*}\left[\mathrm{e}^{-\lambda A_{\tau_{r}}}\right] G(r)=\int_{0}^{\langle\mu, 1\rangle} d r \mathrm{e}^{-\left\langle k_{r} \mu, f\right\rangle} \mathbb{E}\left[\mathrm{e}^{-\lambda \tau_{r}^{(\theta)}}\right] G^{(\theta)}(r)
$$

which ends the proof.

Corollary 6.8. The process $\tilde{\rho}$ under $\mathbb{P}_{\mu, 0}^{*}$ is distributed as $\rho^{(\theta)}$ under $\mathbb{P}_{\mu}^{*}$.

Proof. Let $f \in \mathcal{B}_{+}\left(\mathbb{R}_{+}\right)$bounded. Proposition 6.7 can be re-written as

$$
\int_{0}^{+\infty} \mathrm{e}^{-\lambda t} \mathbb{E}_{\mu, 0}^{*}\left[\mathrm{e}^{-\left\langle\tilde{\rho}_{t}, f\right\rangle} \mathbf{1}_{\{t \leq \tilde{\sigma}\}}\right] d t=\int_{0}^{+\infty} \mathrm{e}^{-\lambda t} \mathbb{E}_{\mu}^{*}\left[\mathrm{e}^{-\left\langle\rho_{t}^{(\theta)}, f\right\rangle} \mathbf{1}_{\left\{t \leq \sigma^{(\theta)}\right\}}\right] d t .
$$

By uniqueness of the Laplace transform, we deduce that, for almost every $t>0$,

$$
\mathbb{E}_{\mu, 0}^{*}\left[\mathrm{e}^{-\left\langle\tilde{\rho}_{t}, f\right\rangle} \mathbf{1}_{\{t \leq \tilde{\sigma}\}}\right]=\mathbb{E}_{\mu}^{*}\left[\mathrm{e}^{-\left\langle\rho_{t}^{(\theta)}, f\right\rangle} \mathbf{1}_{\left\{t \leq \sigma^{(\theta)}\right\}}\right] .
$$


In fact this equality holds for every $r$ by right-continuity. As the Laplace functionals characterize the law of a random measure, we deduce that, for fixed $t>0$, the law of $\tilde{\rho}_{t}$ under $\mathbb{P}_{\mu, 0}^{*}$ is the same as the law of $\rho_{t}^{(\theta)}$ under $\mathbb{P}_{\mu}^{*}$.

The Markov property then give the equality in law for the càd-làg processes $\tilde{\rho}$ and $\rho^{(\theta)}$.

Proof of Theorem 6.1, 0 is recurrent for the Markov càd-làg processes $\tilde{\rho}$ and $\rho^{(\theta)}$. This two processes have no sojourn at 0 , and when killed on the first hitting time of 0 , they have the same law, thanks to Lemma 6.8 From Theorem 4.2 of [9], Section 5, we deduce that $\tilde{\rho}$ under $\mathbb{P}_{\mu, 0}$ is distributed as $\rho^{(\theta)}$ under $\mathbb{P}_{\mu}$.

\section{Property OF THE EXCURSION OF THE PRUNED EXPLORATION PROCESS}

We know, (cf 4], Section VII) that the right continuous inverse, $\left(\tau_{r}, r \geq 0\right)$, of $-I$ is a subordinator with Laplace exponent $\psi^{-1}$. This subordinator has no drift as (2) implies $\lim _{\lambda \rightarrow \infty} \lambda^{-1} \psi^{-1}(\lambda)=0$. We denote by $\pi_{*}$ its Lévy measure: for $\lambda \geq 0$

$$
\psi^{-1}(\lambda)=\int_{(0, \infty)} \pi_{*}(d l)\left(1-\mathrm{e}^{-\lambda l}\right) .
$$

Recall $\mathbb{N}$ is the excursion measure of the exploration process above 0 . If $\sigma$ denotes the duration of the excursion, we have $\mathbb{N}\left[1-\mathrm{e}^{-\lambda \sigma}\right]=\psi^{-1}(\lambda)$. Hence, under $\mathbb{N}, \sigma$ is distributed according to the measure $\pi_{*}$. By decomposing the measure $\mathbb{N}$ w.r.t. the distribution of $\sigma$, we get that $\mathbb{N}[d \mathcal{E}]=\int_{(0, \infty)} \pi_{*}(d r) \mathbb{N}_{r}[d \mathcal{E}]$, where $\left(\mathbb{N}_{r}, r \in(0, \infty)\right)$ is a measurable family of probability measures on the set of excursions such that $\mathbb{N}_{r}[\sigma=r]=1$ for $\pi^{*}$-a.e. $r>0$.

Lemma 7.1. Conditionally on the length of the excursion, the law of the excursion of the pruned exploration process is the law of the excursion of the exploration process.

Proof. From the previous Section, we get that the pruned exploration process $\left(\tilde{\rho}_{t}, t \geq 0\right)$ is distributed according to the law of the exploration process, $\rho^{(\theta)}$, of a Lévy process, $X^{(\theta)}$, with Laplace exponent $\psi^{(\theta)}=\psi(\theta+\cdot)-\psi(\theta)$. In particular the law of the pruned exploration process under the excursion measure is the law of the exploration process $\rho^{(\theta)}$ under the excursion measure.

Let $\sigma^{(\theta)}$ denote the length of the excursion of the exploration process $\rho^{(\theta)}$ under the excursion measure. The following result is known, but since we couldn't give a reference, we shall give a proof at the end of this Section.

Lemma 7.2. For any non-negative measurable function, $G$, on the space of excursions, we have

$$
\mathbb{N}\left[\mathrm{e}^{\psi(\theta) \sigma^{(\theta)}}\left[1-\mathrm{e}^{-G\left(\rho^{(\theta)}\right)}\right]\right]=\mathbb{N}\left[1-\mathrm{e}^{-G(\rho)}\right] .
$$

In particular the distribution of $\rho^{(\theta)}$ under the excursion measure is absolutely continuous w.r.t. to distribution of $\rho$ under the excursion measure, with density given by $\mathrm{e}^{-\sigma \psi(\theta)}$. We deduce that $\pi_{*}^{(\theta)}(d r)=\mathrm{e}^{-r \psi(\theta)} \pi_{*}(d r)$, where $\pi_{*}^{(\theta)}$ is the Lévy measure corresponding to the Laplace exponent $\left(\psi^{(\theta)}\right)^{-1}$. And we have $\pi_{*}(d r)$-a.e., conditionally on the length of the excursion being equal to $r$, the law of the excursion of the pruned exploration process is the law of the excursion of the exploration process.

Recall $\tilde{\sigma}=\int_{0}^{\sigma} \mathbf{1}_{\left\{m_{s}^{(\theta)}=0\right\}} d s$ denotes the length of the excursion of the pruned exploration process. We can compute the joint law of $(\tilde{\sigma}, \sigma)$. This will determine uniquely the law of $\tilde{\sigma}$ conditionally on $\sigma=r$. 
Proposition 7.3. For all non-negative $\gamma, \kappa, \theta$, the value $v$ defined by $v=\mathbb{N}\left[1-\mathrm{e}^{-\psi(\gamma) \sigma-\kappa \tilde{\sigma}}\right]$ is the unique non-negative solution of the equation

$$
\psi(v+\theta)=\kappa+\psi(\gamma+\theta) .
$$

Proof. Using the special Markov property, Theorem [5.2 with $\phi(\mathcal{S})=\psi(\gamma) \sigma$, we have

$$
\begin{aligned}
v=\mathbb{N}\left[1-\mathrm{e}^{-\kappa \tilde{\sigma}-\psi(\gamma) \sigma}\right] & =\mathbb{N}\left[1-\mathrm{e}^{\left.-(\kappa+\psi(\gamma)) \tilde{\sigma}-\psi(\gamma) \int_{0}^{\sigma} \mathbf{1}_{\left\{m_{s} \neq 0\right\}} d s\right]}\right. \\
& =\mathbb{N}\left[1-\mathrm{e}^{-(\kappa+\psi(\gamma)) \tilde{\sigma}-\tilde{\sigma} \int_{(0,+\infty)} \pi(d \ell)\left(1-\mathrm{e}^{-\theta \ell}\right) \mathbb{E}_{\ell}^{*}[1-\exp (-\psi(\gamma) \sigma)]}\right] .
\end{aligned}
$$

Notice that $\sigma$ under $\mathbb{P}_{\ell}^{*}$ is distributed as $\tau_{\ell}$, the first time for which the infimum of $X$, started at 0 , reaches $-\ell$. Since $\tau_{\ell}$ is distributed as a subordinator with Laplace exponent $\psi^{-1}$ at time $\ell$, we have

$$
\mathbb{E}_{\ell}^{*}\left[1-\mathrm{e}^{-\psi(\gamma) \sigma}\right]=\mathbb{E}\left[1-\mathrm{e}^{-\psi(\gamma) \tau_{\ell}}\right]=1-\mathrm{e}^{-\ell \gamma}
$$

and

$\int_{(0,+\infty)} \pi(d \ell)\left(1-\mathrm{e}^{-\theta \ell}\right) \mathbb{E}_{\ell}^{*}\left[1-\mathrm{e}^{-\psi(\gamma) \sigma}\right]=\int_{(0,+\infty)} \pi(d \ell)\left(1-\mathrm{e}^{-\theta \ell}\right)\left(1-\mathrm{e}^{-\gamma \ell}\right)=\psi^{(\theta)}(\gamma)-\psi(\gamma)$

We get

$$
v=\mathbb{N}\left[1-\mathrm{e}^{-\tilde{\sigma}\left(\kappa+\psi^{(\theta)}(\gamma)\right)}\right]=\psi^{(\theta)^{-1}}\left(\kappa+\psi^{(\theta)}(\gamma)\right) .
$$

Using Corollary 6.2 and definition (17) of $\psi^{(\theta)}$, we have $\psi(v+\theta)=\kappa+\psi(\gamma+\theta)$. Since $\psi$ is increasing and continuous, this equation has only one solution.

Proof of Lemma 7.2. Since an excursion of the exploration process above 0 can be recovered from an excursion of the process $X$ above its minimum. We shall prove the Lemma in the latter case.

Let $\theta>0$. We set $X^{(\theta)}=\left(X_{t}^{(\theta)}, t \geq 0\right)$ the Lévy process with Laplace exponent $\psi^{(\theta)}$. Notice that $\left(\mathrm{e}^{-\theta X_{t}-t \psi(\theta)}, t \geq 0\right)$ is a martingale w.r.t. the natural filtration generated by $X$, $\left(\mathcal{H}_{t}, t \geq 0\right)$. We define a new probability by

$$
d \mathbb{P}_{\mid \mathcal{H}_{t}}^{(\theta)}=\mathrm{e}^{-\theta X_{t}-t \psi(\theta)} d \mathbb{P}_{\mid \mathcal{H}_{t}}
$$

The law of $\left(X_{u}, u \in[0, t]\right)$ under $\mathbb{P}^{(\theta)}$ is the law of $\left(X_{u}^{(\theta)}, u \in[0, t]\right)$. Therefore, we have for any non-negative measurable function on the path space

$$
\mathbb{E}\left[F\left(X_{\leq t}^{(\theta)}\right) \mathrm{e}^{\theta X_{t}^{(\theta)}+t \psi(\theta)}\right]=\mathbb{E}\left[F\left(X_{\leq t}\right)\right] .
$$

We define $-I_{t}^{(\theta)}=-\inf _{u \in[0, t]} X_{u}^{(\theta)}$, and $\tau^{(\theta)}$ its right-continuous inverse. In particular, it is a subordinator of Laplace exponent $\psi^{(\theta)^{-1}}$. Since $\psi^{(\theta)^{-1}}(\lambda)=\psi^{-1}(\lambda+\psi(\theta))-\theta$, we have

$$
\mathbb{E}\left[\mathrm{e}^{-\lambda \tau_{r}^{(\theta)}}\right]=\mathrm{e}^{-r\left[\psi^{-1}(\lambda+\psi(\theta))-\theta\right]}
$$

Furthermore, this equality holds for $\lambda \geq-\psi(\theta)$. With $\lambda=-\psi(\theta)$, we get $\mathbb{E}\left[\mathrm{e}^{\psi(\theta) \tau_{r}^{(\theta)}}\right]=\mathrm{e}^{\theta r}$.

From (41), we get that the process $\left(Q_{t}, t \geq 0\right)$, where $Q_{t}=\mathrm{e}^{\theta X_{t}^{(\theta)}+t \psi(\theta)}$ is a martingale. Since $M_{\tau_{r}^{(\theta)}}=\mathrm{e}^{-\theta r+\psi(\theta) \tau_{r}^{(\theta)}}$ is integrable and $\mathbb{E}\left[M_{\tau_{r}^{(\theta)}}\right]=1$, we deduce from (41) that

$$
\mathbb{E}\left[F\left(X_{\leq \tau_{r}^{(\theta)}}^{(\theta)}\right) \mathrm{e}^{-\theta r+\psi(\theta) \tau_{r}^{(\theta)}}\right]=\mathbb{E}\left[F\left(X_{\leq \tau_{r}}\right)\right]
$$


Let $\mathcal{E}_{i}=\left(X_{t+\alpha_{i}}-I_{\alpha_{i}}, t \in\left[\alpha_{i}, \alpha_{i}+\sigma_{i}\right]\right), i \in I$, be the excursions of $X$ above its minimum, up to time $\tau_{r}$. With $F$ such that $F\left(X_{\leq \tau_{r}}\right)=\mathrm{e}^{-\sum_{i \in I} G\left(\mathcal{E}_{i}\right)}$, we get

$$
\mathbb{E}\left[F\left(X_{\leq \tau_{r}}\right) \mathrm{e}^{-\lambda \tau_{r}}\right]=\mathrm{e}^{-r \mathbb{N}\left[1-\mathrm{e}^{-G(\mathcal{E})-\lambda \sigma}\right]} .
$$

We deduce from (42) that

$$
\mathrm{e}^{-\theta r} \mathrm{e}^{-r \mathbb{N}\left[1-\mathrm{e}^{-G\left(\mathcal{E}^{(\theta)}\right)+\psi(\theta) \sigma^{(\theta)}}\right]}=\mathrm{e}^{-r \mathbb{N}\left[1-\mathrm{e}^{-G(\mathcal{E})}\right]},
$$

where $\mathcal{E}^{(\theta)}$ is an excursion of $X^{(\theta)}$ above its minimum, that is

$$
\mathbb{N}\left[1-\mathrm{e}^{-G\left(\mathcal{E}^{(\theta)}\right)+\psi(\theta) \sigma^{(\theta)}}\right]=\mathbb{N}\left[1-\mathrm{e}^{-G(\mathcal{E})}\right]-\theta .
$$

Subtracting $\mathbb{N}\left[1-\mathrm{e}^{\psi(\theta) \sigma^{(\theta)}}\right]=-\theta$, in the above equality, we get

$$
\mathbb{N}\left[\mathrm{e}^{\psi(\theta) \sigma^{(\theta)}}\left[1-\mathrm{e}^{-G\left(\mathcal{E}^{(\theta)}\right)}\right]\right]=\mathbb{N}\left[1-\mathrm{e}^{-G(\mathcal{E})}\right] .
$$

\section{LiNK BETWEEN LÉvy SNAKE AND FRAGMENTATION PROCESSES AT NODES}

We define the fragmentation process. Let $\mathcal{S}=(\rho, M)$ be a Lévy Poisson snake. Recall definition of $m^{(\theta)}$ at the end of Section [3 For fixed $\theta>0$, let us consider the following equivalence relation $\mathcal{R}_{\theta}$ on $[0, \sigma]$, defined under $\mathbb{N}$ or $\mathbb{N}_{\sigma}$ (see definition in Section [7) by:

$$
s \mathcal{R}_{\theta} t \Longleftrightarrow m_{s}^{(\theta)}\left(\left[H_{s, t}, H_{s}\right]\right)=m_{t}^{(\theta)}\left(\left[H_{s, t}, H_{t}\right]\right)=0,
$$

where $H_{s, t}=\inf _{u \in[s, t]} H_{u}$ (recall definition (30). Intuitively, two points $s$ and $t$ belongs to the same class of equivalence (i.e. the same fragment) at time $\theta$, if there is no cut on their lineage down to their most recent common ancestor (that is $m_{s}^{(\theta)}$ put no mass on $\left[H_{s, t}, H_{s}\right]$ nor $m_{t}^{(\theta)}$ on $\left.\left[H_{s, t}, H_{t}\right]\right)$. Notice cutting occurs on branching points, that is at node of the CRT. Each node of the CRT correspond to a jump of the underlying Lévy process $X$. The cutting times are, conditionally on the CRT, independent exponential random times, with parameter equal to the jump of the corresponding node.

Let us index the different equivalent classes in the following way: For any $s \leq \sigma$, let us define $H_{s}^{0}=0$ and recursively for $k \in \mathbb{N}$,

$$
H_{s}^{k+1}=\inf \left\{u \geq 0 \mid m_{s}^{\theta}\left(\left(H_{s}^{k}, u\right]\right)>0\right\},
$$

with the usual convention $\inf \emptyset=+\infty$. We set

$$
K_{s}=\sup \left\{j \in \mathbb{N}, H_{s}^{j}<+\infty\right\} .
$$

Remark 8.1. Notice that we have $K_{s}=\infty$ if $M_{s}(\cdot,[0, \theta])$ has infinitely many atoms. By construction of $M$ using Poisson point measures, this happens $\mathbb{N}[d \mathcal{S}] d s$-a.e., if and only if the intensity measure $\rho_{s}+\eta_{s}$ is infinite. Since $\mathbb{N}[d \mathcal{S}]$-a.e., $\rho$ and $\eta$ are finite measure valued process, we get that $\mathbb{N}[d \mathcal{S}]$-a.e., $K_{s}<\infty$.

Let us remark that $s \mathcal{R}_{\theta} t$ implies $K_{s}=K_{t}$. We denote, for any $j \in \mathbb{N},\left(R^{j, k}, k \in J_{j}\right)$ the family of equivalent classes with positive Lebesgue measure such that $K_{s}=j$. For $j \in \mathbb{N}$, $k \in J_{j}$ we set

$$
A_{t}^{j, k}=\int_{0}^{t} \mathbf{1}_{\left\{s \in R^{j, k}\right\}} d s \quad \text { and } \quad C_{t}^{j, k}=\inf \left\{u \geq 0, A_{u}^{j, k}>t\right\}
$$


with the convention $\inf \emptyset=\sigma$. And we define the corresponding Lévy snake, $\tilde{\mathcal{S}}^{j, k}=$ $\left(\tilde{\rho}^{j, k}, \tilde{M}^{j, k}\right)$ by: for every $f \in \mathcal{B}_{+}\left(\mathbb{R}_{+}\right), \varphi \in \mathcal{B}_{+}\left(\mathbb{R}_{+} \times \mathbb{R}_{+}\right), t \geq 0$,

$$
\begin{aligned}
\left\langle\tilde{\rho}_{t}^{j, k}, f\right\rangle & =\int_{\left(H_{C_{0}^{j, k}},+\infty\right)} f\left(x-H_{C_{0}^{j, k}}\right) \rho_{C_{t}^{j, k}}(d x) \\
\left\langle\tilde{M}_{t}^{j, k}, \varphi\right\rangle & =\int_{\left(H_{\left.C_{0}^{j, k},+\infty\right) \times(\theta,+\infty)}\right.} \varphi\left(x-H_{C_{0}^{j, k}}, v-\theta\right) M_{C_{t}^{j, k}}(d x, d v) .
\end{aligned}
$$

Let $\tilde{\sigma}^{j, k}=A_{\infty}^{j, k}$ be the length of the excursion $\tilde{\mathcal{S}}^{j, k}$. Since $K_{s}<\infty \mathbb{N}[d \mathcal{S}] d s$-a.e. (Remark 8.1), the family $\left(\tilde{\sigma}^{j, k} j \in \mathbb{N}, k \in J_{j}\right)$ gives all the equivalent classes with positive Lebesgue measure.

Remark 8.2. In view of the next Section we introduce the set $\mathcal{L}^{(\theta)}=\left(\tilde{\rho}^{(j, k)}, j \in \mathbb{N}, k \in J_{j}\right)$ of fragments of Lévy snake as well as the the set $\mathcal{L}^{(\theta-)}$ defined similarly but for the equivalence relation where $\mathcal{R}_{\theta}$ in (43) is replaced by $\mathcal{R}_{\theta-}$ defined as

$$
s \mathcal{R}_{\theta-} t \Longleftrightarrow M_{s}\left(\left[H_{s, t}, H_{s}\right] \times(0, \theta)\right)=M_{s}\left(\left[H_{s, t}, H_{t}\right] \times(0, \theta)\right)=0 .
$$

Notice that $m_{s}^{(\theta)}(\cdot)=M_{s}(\cdot,(0, \theta])$. So the two equivalence relations are equal $\mathbb{N}$-a.e. for fixed $\theta$, but may differ if $M$ has an atom in $\{\theta\} \times \mathbb{R}_{+}$.

Let us denote by $\Lambda^{\theta}=\left(\Lambda_{1}^{\theta}, \Lambda_{2}^{\theta}, \ldots\right)$ the sequence of positive Lebesgue measures of the equivalent classes of $\mathcal{R}_{\theta},\left(\tilde{\sigma}^{j, k}, j \in \mathbb{N}, k \in J_{j}\right)$, ranked in decreasing order. Notice this sequence is at most countable. If it is finite, we complete the sequence with zeros, so that $\mathbb{N}$-a.s. and $\mathbb{N}_{\sigma}$-a.s.

$$
\Lambda^{\theta} \in \mathcal{S}^{\downarrow}=\left\{\left(x_{1}, x_{2}, \ldots\right), x_{1} \geq x_{2} \geq \cdots \geq 0, \sum x_{i} \leq \infty\right\}
$$

For $\pi^{*}(d \sigma)$-a.e. $\sigma>0$, let $\mathrm{P}_{\sigma}$ denote the law of $\left(\Lambda^{\theta}, \theta \geq 0\right)$ under $\mathbb{N}_{\sigma}$. (The law, $\mathbb{N}_{r}$, of $\mathcal{S}$ conditionally on the length of the excursion, $\sigma$, being equal to $r$ has been defined in the previous Section.) By convention $\mathrm{P}_{0}$ is the Dirac mass at $(0,0, \ldots) \in \mathcal{S}^{\downarrow}$.

Theorem 8.3. For $\pi_{*}(d r)$-almost any $r$, under $\mathrm{P}_{r}$, the process $\Lambda=\left(\Lambda^{\theta}, \theta \geq 0\right)$ is a $\mathcal{S}^{\downarrow}$ valued fragmentation process. More precisely, the law under $\mathrm{P}_{r}$ of the process $\left(\Lambda^{\theta+\theta^{\prime}}, \theta^{\prime} \geq 0\right)$ conditionally on $\Lambda^{\theta}=\left(\Lambda_{1}, \Lambda_{2}, \ldots\right)$ is given by the decreasing reordering of independents processes of respective law $\mathrm{P}_{\Lambda_{1}}, \mathrm{P}_{\Lambda_{2}}, \ldots$

Remark 8.4. We get a self-similar fragmentation when $\psi(\lambda)=\lambda^{\alpha}$, see Corollary 9.3. This particular case was already studied in [18].

Remark 8.5. We may get rid of the " $\pi_{*}(d r)$-almost any $r$ " and have the theorem for any positive $r$ if we have a regular version of the family of conditional probability laws $\left(\mathbb{N}_{r}, r>0\right)$. This is for instance the case when the Lévy process is stable (for which it is possible to construct the measure $\mathbb{N}_{r}$ from $\mathbb{N}_{1}$ by a scaling property) or when we may construct this family via a Vervaat's transform of the Lévy bridge (see [16]).

The proof of the Proposition is a consequence of Lemma 8.6 and the fact that $\mathbb{N}(\cdot)=$ $\int_{(0,+\infty)} \pi_{*}(d r) \mathbb{N}_{r}(\cdot)$ which implies that the result of Lemma 8.6 holds $\mathbb{N}_{r}$-a.s. for $\pi_{*}(d r)$ almost every $r$.

Lemma 8.6. Under $\mathbb{N}$, the law of the family $\left(\tilde{\mathcal{S}}^{j, k}, j \in \mathbb{N}, k \in J_{j}\right)$, conditionally on $\left(\tilde{\sigma}^{j, k}, j \in\right.$ $\left.\mathbb{N}, k \in J_{j}\right)$, is the law of independent Lévy Poisson snakes, and the conditional law of $\tilde{\mathcal{S}}^{j, k}$ is $\mathbb{N}_{\tilde{\sigma}^{j, k}}$. 
Proof. For $j=0$, notice that $J_{0}$ has only one element, say 0 . And $\tilde{\mathcal{S}}^{0,0}$ is just the Lévy snake, $\tilde{\mathcal{S}}$, defined in Section [5] Of course, we have $\tilde{\sigma}^{0,0}=\tilde{\sigma}$. From the special Markov property (Theorem [5.2) and Proposition 3.5] we deduce that conditionally on $\tilde{\sigma}^{0,0}, \tilde{\mathcal{S}}^{0,0}$ and the family $\left(\mathcal{S}^{i}, i \in I\right)$ of excursions of $\mathcal{S}$ out of $\left\{s \geq 0 ; m_{s}^{\theta}=0\right\}$ (as defined in Section 5) are independent.

From Corollary [6.2 and Lemma 7.1 for the exploration process and Proposition [3.5] for the underlying Poisson process, we deduce that, conditionally on $\tilde{\sigma}^{0,0}, \tilde{\mathcal{S}}^{0,0}$ is distributed according to $\mathbb{N}_{\tilde{\sigma}^{0,0}}$.

Furthermore, from the special Markov property (Theorem [5.2), the conditional law of $\mathcal{S}^{i}$ is given by $\mathrm{N}$, defined in (23). Now we give a Poisson decomposition of the measure $\mathrm{N}$.

For $\mathcal{S}^{\prime}=\left(\rho^{\prime}, M^{\prime}\right)$ distributed according to $\mathrm{N}$, we consider $\left(\alpha_{l}^{\prime}, \beta_{l}\right)_{l \in I^{\prime}}$ the excursion intervals of the Lévy Poisson snake, $\mathcal{S}^{\prime}$, out of $\left\{H_{s}^{\prime}=0\right\}$. For $l \in I^{\prime}$, we set $\mathcal{S}^{\prime l}=\left(\rho^{\prime l}, M^{\prime l}\right)$ where for $s \geq 0$,

$$
\begin{aligned}
\rho_{s}^{\prime}(d r) & =\rho_{\left(s+\alpha_{l}^{\prime}\right) \wedge \beta_{l}^{\prime}}^{\prime}(d r) \mathbf{1}_{(0,+\infty)}(r), \\
M_{s}^{\prime l}(d r, d v) & =M_{\left(s+\alpha_{l}^{\prime}\right) \wedge \beta_{l}^{\prime}}^{\prime}(d r, d v) \mathbf{1}_{(0,+\infty)}(r) .
\end{aligned}
$$

Let us remark that in the above definition $\rho^{\prime l}$ and $M^{\prime l}$ don't have mass at $\{0\}$ and $\{0\} \times \mathbb{R}_{+}$.

As a direct consequence of the Poisson decomposition of $\mathbb{P}_{\ell}^{*}$ (see Lemma 3.3), we get the following Lemma.

Lemma 8.7. Under $\mathrm{N}$, the point measure $\sum_{i^{\prime} \in I^{\prime}} \delta_{\mathcal{S}^{i^{\prime}}}$ is a Poisson point measure with intensity $C_{\theta} \mathbb{N}(d \mathcal{S})$ where $C_{\theta}=\int_{(0, \infty)}\left(1-\mathrm{e}^{-\theta \ell}\right) \ell \pi(d \ell)=\psi^{\prime}(\theta)-\psi^{\prime}(0)$.

By this Poisson representation, each process $\mathcal{S}^{i}$ is composed of i.i.d. excursions of law $\mathbb{N}$. Thus we get, conditionally on $\tilde{\sigma}^{0,0}$, a family $\left(\mathcal{S}^{1, k}, k \in J_{1}\right)$ of i.i.d. excursions distributed as the atoms of a Poisson point measure with intensity $\tilde{\sigma}^{0,0} C_{\theta} \mathbb{N}$. Now, we can repeat the above arguments for each excursion $\mathcal{S}^{1, k}, k \in J_{1}$ : so that conditionally on $\tilde{\sigma}^{0,0}$, we can

- check that $\tilde{\mathcal{S}}^{1, k}$ is built from $\mathcal{S}^{1, k}$ as $\tilde{\mathcal{S}}$ from $\mathcal{S}$ in Section 15 ,

- get a family $\left(\mathcal{S}^{2, k^{\prime}, k}, k^{\prime} \in J_{2}^{k}\right)$, which are, conditionally on $\tilde{\sigma}^{1, k}$, distributed as the atoms of a Poisson point measure with intensity $\tilde{\sigma}^{1, k} C_{\theta} \mathbb{N}$. and are independent of $\tilde{\mathcal{S}}^{1, k}$.

If we set $J_{2}=\cup_{k \in J_{1}} J_{2}^{k} \times\{k\}$, we get that conditionally on $\tilde{\sigma}^{0,0}$, and $\left(\tilde{\sigma}^{1, k}, k \in J_{1}\right)$,

- the excursions $\tilde{\mathcal{S}}^{0,0}$ and $\left(\tilde{\mathcal{S}}^{1, k}, k \in J_{1}\right)$, are independent,

- $\tilde{\mathcal{S}}^{i, k}$ is distributed as $\mathbb{N}_{\tilde{\sigma}^{j, k}}$, for $j \in\{0,1\}, k \in J_{j}$,

- $\left(\mathcal{S}^{2, k^{\prime}}, k^{\prime} \in J_{2}\right)$, are distributed as the atoms of a Poisson point measure with intensity $\sum_{k \in J_{1}} \tilde{\sigma}^{1, k} C_{\theta} \mathbb{N}$, and are independent of $\tilde{\mathcal{S}}^{0,0}$ and $\left(\tilde{\mathcal{S}}^{1, k}, k \in J_{1}\right)$.

Eventually, the result follows by induction.

Now we check there is no loss of mass during the fragmentation.

Proposition 8.8. For $\pi_{*}(d r)$ almost every $r, \mathrm{P}_{r}$-a.s., for every $\theta \geq 0, \sum_{i=1}^{+\infty} \Lambda_{i}^{\theta}=r$. 
Proof. Let $\theta>0$. We use the notations of the proof of Theorem 8.3 and of Lemma 8.6. For $n \in \mathbb{N}$, we have $\mathbb{N}$-a.e.

$$
\sigma=\sum_{k=0}^{n} \sum_{j \in J_{k}} \tilde{\sigma}^{j, k}+\int_{0}^{\sigma} \mathbf{1}_{\left\{K_{s} \geq n+1\right\}} d s
$$

By monotone convergence, we deduce from Remark 8.1 that we get as $n \rightarrow+\infty$ that $\mathbb{N}$-a.e.

$$
\sigma=\sum_{k=0}^{\infty} \sum_{j \in J_{k}} \tilde{\sigma}^{j, k}
$$

As the decreasing reordering of $\left(\tilde{\sigma}^{j, k}, j \in \mathbb{N}, k \in J_{j}\right)$ is $\Lambda^{\theta}$, we get that $\mathbb{N}$-a.e. $\sum_{i=1}^{+\infty} \Lambda_{i}^{\theta}=\sigma$. As the sequence $\left(\sum_{i=1}^{\infty} \Lambda_{i}^{\theta}, \theta \geq 0\right)$ is non increasing, we deduce that the previous equality holds for any $\theta>0, \mathbb{N}$-a.e.

Here again the result for $P_{r}$ is deduced from the one under $\mathbb{N}$.

\section{Dislocation measures}

Let $\lambda(\theta)$ be the mass of a tagged fragment at time $\theta$ of the fragmentation process $\Lambda$ defined in Theorem 8.3 (typically the fragment or the equivalent class which contains 0 ). A dislocation of this fragment occurs when $\lambda(\theta)$ has a jump. Let $\mathcal{T}_{0}$ be the set of time jumps for $\lambda$. Recall $\mathcal{S}^{\downarrow}$ denote the set of non-negative non-increasing sequence $\left(x_{i}, i \in \mathbb{N}^{*}\right)$ such that $\sum_{i \geq 1} x_{i}<\infty$. For $\theta^{\prime} \in \mathcal{T}_{0}$, let $x\left(\theta^{\prime}\right)=\left(x_{i}\left(\theta^{\prime}\right), i \in \mathbb{N}^{*}\right) \in \mathcal{S}^{\downarrow}$, the masses of the fragments resulting of the dislocation at time $\theta^{\prime}$. Following the Remark after Theorem 3 in [6], we call the random point measure

$$
\delta(d \theta, d x)=\sum_{\theta^{\prime} \in \mathcal{T}_{0}} \delta_{\theta^{\prime}, x\left(\theta^{\prime}\right)}(d \theta, d x)
$$

the dislocation process of the fragmentation (or dislocation process of the $\psi$-CRT fragmentation at nodes). Of course, since there is no erosion, that is the total length is constant cf. Proposition 8.8 $\lambda\left(\theta^{\prime}-\right)=\sum_{i \geq 1} x_{i}\left(\theta^{\prime}\right)$.

For self-similar fragmentation with with index $\gamma$ and no erosion, there exists a measure $\nu_{1}$ on $\mathcal{S}_{1}^{\downarrow}=\left\{x \in \mathcal{S}^{\downarrow} ; \sum_{i \geq 1} x_{i}=1\right\}$, called the dislocation measure, such that the dislocation process is a point process with intensity $\mathbf{1}_{\{\lambda(\theta-)>0\}} \nu_{\lambda(\theta-)}(d x) d \theta$, where the measures $\left(\nu_{r}, r>0\right)$ are defined by

$$
\int_{\mathcal{S}_{r}^{\downarrow}} F(x) \nu_{r}(d x)=r^{\gamma} \int_{\mathcal{S}_{1}^{\downarrow}} F(r x) \nu_{1}(d x),
$$

and the equality hold for any non-negative measurable function on $\mathcal{S}^{\downarrow}$. We refer to [6] for the proof of this result and to [13] for the definition of intensity of a random point measure.

In order to give the corresponding dislocation measures for the $\psi$-CRT fragmentation at nodes, we need to consider $\left(\Delta S_{t}, t \geq 0\right)$ the jumps of a subordinator $S$ with Laplace exponent $\psi^{-1}$. Let $\mu$ the measure on $\mathbb{R}_{+} \times \mathcal{S}^{\downarrow}$ such that for any non-negative measurable function, $F$, on $\mathbb{R}_{+} \times \mathcal{S}^{\downarrow}$,

$$
\int_{\mathbb{R}_{+} \times \mathcal{S} \downarrow} F(r, x) \mu(d r, d x)=\int \pi(d v) \mathbb{E}\left[F\left(S_{v},\left(\Delta S_{t}, t \leq v\right)\right)\right]
$$


where $\left(\Delta S_{t}, t \leq v\right)$ has to be understood as the family of jumps of the subordinator up to time $v$ ranked in decreasing size.

Intuitively, $\mu$ is the law of $S_{T}$ and the jumps of $S$ up to time $T$, where $T$ and $S$ are independent, and $T$ is distributed according to the infinite measure $\pi$. Recall $\pi_{*}$ is the "law" of $\sigma$ under $\mathbb{N}$ (this is the Lévy measure associated to the Laplace exponent $\psi^{-1}$ ).

Theorem 9.1. The dislocation process of the $\psi$-CRT fragmentation at nodes, is under $\mathbb{N} a$ point process with intensity $\mathbf{1}_{\{\lambda(\theta-)>0\}} \nu_{\lambda(\theta-)}(d x) d \theta$, where $\lambda(\theta-)=\sum_{i \geq 1} x_{i}(\theta)$ is the mass of the fragment just before $\theta$. And the family of dislocation measure $\left(\nu_{r}, r>0\right)$ on $\mathcal{S}^{\downarrow}$ is the result of the disintegration of $r \mu(d r, d x)$ w.r.t. $\pi_{*}(d r)$ :

$$
r \mu(d r, d x)=\nu_{r}(d x) \pi_{*}(d r) .
$$

Notice that (46) implies that $\pi^{*}(d r)$-a.e. $\nu_{r}(d x)$-a.e. $\sum_{i \in \mathbb{N}^{*}} x_{i}=r$, where $x=\left(x_{i}, i \in \mathbb{N}^{*}\right)$. The dislocation measure $\nu_{r}$ describe the dislocation of a fragment of size $r$.

Remark 9.2. Either from Lemma 7.1 or directly, it is easy to check that the dislocation measure of the fragmentation at nodes associated to $\psi^{(\theta)}$ (see (17)),$\left(\nu_{r}^{(\theta)}, r>0\right)$, is equal to $\left(\nu_{r}, r>0\right), \pi_{*}(d r)$-a.e.

The next Sections are devoted to the proof of the Theorem. In Section 9.1, we give an other representation of the fragmentation following ideas in [1, 3] developed for $\psi(\lambda)=\lambda^{2}$. In Section 9.2, we explain how to compute the intensity of the dislocation process. And we perform the computation in Section 9.3. This will end the proof of the Theorem.

For the $\lambda^{\alpha}$-CRT (with $\alpha \in(1,2)$ ), thanks to scaling properties, the corresponding fragmentation is self similar with index $1 / \alpha$, and we can recover the result of $[18$.

Corollary 9.3. For the $\lambda^{\alpha}$-CRT fragmentation at nodes, the fragmentation is self-similar, with index $1 / \alpha$, that is (45) holds with $\gamma=1 / \alpha$. And the dislocation measure $\nu_{1}$ on $\mathcal{S}_{1}^{\downarrow}$ is s.t.

$$
\int F(x) \nu_{1}(d x)=\frac{\alpha(\alpha-1) \Gamma([\alpha-1] / \alpha)}{\Gamma(2-\alpha)} \mathbb{E}\left[S_{1} F\left(\left(\Delta S_{t} / S_{1}, t \leq 1\right)\right)\right],
$$

holds for any non-negative measurable function, $F$, on $\mathcal{S}_{1}^{\downarrow}$, where $\left(\Delta S_{t}, t \geq 0\right)$ are the jumps of a stable subordinator $S=\left(S_{t}, t \geq 0\right)$ of Laplace exponent $\psi^{-1}(\lambda)=\lambda^{1 / \alpha}$, ranked by decreasing size.

Proof. For $\psi(\lambda)=\lambda^{\alpha}$, we get $\pi(d r)=\alpha(\alpha-1) \Gamma(2-\alpha)^{-1} r^{-1-\alpha} d r$ as well as $\pi_{*}(d r)=$ $[\alpha \Gamma([\alpha-1] / \alpha)]^{-1} r^{-(1+\alpha) / \alpha} d r$. In particular, we have for a non-negative measurable function, $F$, defined on $\mathbb{R}_{+} \times \mathcal{S}_{1}^{\downarrow}$,

$$
\begin{aligned}
\int F(r, x) r \mu(d r, d x) & =\mathbb{E}\left[\int \pi(d v) S_{v} F\left(S_{v},\left(\Delta S_{t}, t \leq v\right)\right)\right] \\
& =\frac{\alpha(\alpha-1)}{\Gamma(2-\alpha)} \mathbb{E}\left[\int \frac{d v}{v^{1+\alpha}} S_{v} F\left(S_{v},\left(\Delta S_{t}, t \leq v\right)\right)\right] \\
& =\frac{\alpha(\alpha-1)}{\Gamma(2-\alpha)} \mathbb{E}\left[\int \frac{d v}{v} S_{1} F\left(v^{\alpha} S_{1}, v^{\alpha} S_{1}\left(\Delta S_{t} / S_{1}, t \leq 1\right)\right)\right] \\
& =\frac{\alpha-1}{\Gamma(2-\alpha)} \int \mathbb{E}\left[S_{1} F\left(y, y\left(\Delta S_{t} / S_{1}, t \leq 1\right)\right)\right] \frac{d y}{y},
\end{aligned}
$$

where we used the scaling property of $S$, that is $\left(\Delta S_{t}, t \leq r\right)$ is distributed as $\left(r^{\alpha} \Delta S_{t}, t \leq 1\right)$, for the third equality, and the change of variable $y=v^{\alpha} S_{1}$ for the fourth equality. From 
Theorem 9.1, we have that

$$
\int \frac{1}{\alpha \Gamma([\alpha-1] / \alpha)} \frac{d r}{r^{(1+\alpha) / \alpha}} \nu_{r}(d x) F(r, x)=\int \frac{\alpha-1}{\Gamma(2-\alpha)} \mathbb{E}\left[S_{1} F\left(y,\left(y \Delta S_{t}, t \leq 1\right)\right)\right] \frac{d y}{y} .
$$

This implies that for a.a. $r>0$,

$$
\int \nu_{r}(d x) F(x)=\frac{\alpha(\alpha-1) \Gamma([\alpha-1] / \alpha)}{\Gamma(2-\alpha)} r^{1 / \alpha} \mathbb{E}\left[S_{1} F\left(r\left(\Delta S_{t} / S_{1}, t \leq 1\right)\right)\right],
$$

and thus $\int \nu_{r}(d x) F(x)=r^{1 / \alpha} \int \nu_{1}(d x) F(r x)$, with

$$
\int \nu_{1}(d x) F(x)=\frac{\alpha(\alpha-1) \Gamma([\alpha-1] / \alpha)}{\Gamma(2-\alpha)} \mathbb{E}\left[S_{1} F\left(\left(\Delta S_{t} / S_{1}, t \leq 1\right)\right)\right] .
$$

9.1. An other representation of the fragmentation. Following the ideas in [1, 3], we give an other representation of the fragmentation process described in Section 8 using a Poisson point measure under the epigraph of the height process.

We consider a fragmentation process, as time $\theta$ increases, of the CRT, by cutting at nodes (set of points $(s, a)$ such that $\kappa_{s}(\{a\})>0$, where $\kappa$ is defined in ([6]). More precisely, we consider, conditionally on the CRT or equivalently on the exploration process $\rho$, a Poisson point process, $\mathcal{Q}(d \theta, d s, d a)$ under the epigraph of $H$, with intensity $d \theta q_{\rho}(d s, d a)$, where

$$
q_{\rho}(d s, d a)=\frac{d s \kappa_{s}(d a)}{d_{s, a}-g_{s, a}}
$$

with $d_{s, a}=\sup \left\{u \geq s, \min \left\{H_{v}, v \in[s, u]\right\} \geq a\right\}$ and $g_{s, a}=\inf \left\{u \leq s, \min \left\{H_{v}, v \in[u, s]\right\} \geq\right.$ $a\}$. (The set $\left[g_{s, a}, d_{s, a}\right] \subset[0, \sigma]$ represent the individuals who have a common ancestor with the individual $s$ after or at generation $a$.)

Notice that from this representation, the cutting times of the nodes are, conditionally on the CRT, independent exponential random time, and their parameter is equal to the mass of the node (defined as the mass of $\kappa$ or equivalently as the value of the jump of $X$ corresponding to the given node).

We say two points $s, s^{\prime} \in[0, \sigma]$ belongs to the same fragment at time $\theta$, if there is no cut on their lineage down to their most recent common ancestor $H_{s, s^{\prime}}$ : that is for $v=s$ and $v=s^{\prime}$,

$$
\int \mathbf{1}_{\left[H_{s, s^{\prime}}, H_{v}\right]}(a) \mathbf{1}_{\left[g_{v, a}, d_{v, a}\right]}(u) \mathcal{Q}([0, \theta], d u, d a)=0 .
$$

This define an equivalence relation, and we call fragment an equivalent class. Let $\Lambda^{\theta}$ be the sequences of Lebesgue measures of the corresponding equivalent classes ranked in decreasing order.

It is clear that conditionally on the CRT, the process $\left(\Lambda^{\theta}, \theta \geq 0\right)$ as the same distribution as the fragmentation process defined in Section [8. Roughly speaking, in Section 3 (which leads to the fragmentation of Section 8) we mark the node as they appear: that is, for a given level $a$, the node $\left\{s ; \kappa_{s}(\{a\})>0\right\}$ is marked at $g_{s, a}$. Whereas in this Section the same node is marked uniformly on $\left[g_{s, a}, d_{s, a}\right]$. In both case, the cutting times of the nodes are, conditionally on the CRT, independent exponential random time, and their parameter is equal to the mass of the node (defined as the common value of $\kappa_{u}(\{a\})$ for $u \in\left\{s ; \kappa_{s}(\{a\})>0\right\}$, or equivalently as the value of the jump of $X$ corresponding to the given node). 
Now, we define the fragments of the Lévy snake corresponding to the cutting of $\rho$ according to the measure $q_{\rho}$. For $(s, a)$ chosen according to the measure $q_{\rho}(d s, d a)$, we can define the following Lévy snake fragments $\left(\rho^{i}, i \in \tilde{I}\right)$ of $\rho$ by considering

- the open intervals of excursion after $s$ of $H$ above level $a:\left(\left(\alpha_{i}, \beta_{i}\right), i \in \tilde{I}_{+}\right)$, which are such that $\alpha_{i}>s, H_{\alpha_{i}}=H_{\beta_{i}}=a$, and for $s^{\prime} \in\left(\alpha_{i}, \beta_{i}\right)$ we have $H_{s^{\prime}}>a$ and $H_{s^{\prime}, s}=a$ (recall definition (3) );

- the open intervals of excursion before $s$ of $H$ above level $a:\left(\left(\alpha_{i}, \beta_{i}\right), i \in \tilde{I}_{-}\right)$, which are such that $\beta_{i}<s, H_{\alpha_{i}}=H_{\beta_{i}}=a$, and for $s^{\prime} \in\left(\alpha_{i}, \beta_{i}\right)$ we have $H_{s^{\prime}}>a$ and $H_{s^{\prime}, s}=a$;

- the excursion, $i_{s}$, of $H$ above level $a$ that straddle $s:\left(\alpha_{i_{s}}, \beta_{i_{s}}\right)$, which is such that $\alpha_{i_{s}}<s<\beta_{i_{s}}, H_{\alpha_{i_{s}}}=H_{\beta_{i_{s}}}=a$, and for $s^{\prime} \in\left(\alpha_{i_{s}}, \beta_{i_{s}}\right)$ we have $H_{s^{\prime}}>a$ and $H_{s^{\prime}, s}=a$;

- the excursion, $i_{0}$, of $H$ under level $a:\left\{s \in[0, \sigma] ; H_{s^{\prime}, s}<a\right\}=\left[0, \alpha_{i_{0}}\right) \cup\left(\beta_{i_{0}}, \sigma\right]$.

For $i \in \tilde{I}_{+} \cup \tilde{I}_{-} \cup\left\{i_{s}\right\}$, we set $\rho^{i}=\left(\rho_{s}^{i}, s \geq 0\right)$ where

$$
\int f(r) \rho_{s}^{i}(d r)=\int f(r-a) \mathbf{1}_{\{r>a\}} \rho_{\left(\alpha_{i}+s\right) \wedge \beta_{i}}(d r)
$$

for $f \in \mathcal{B}_{+}(\mathbb{R})$. For $i_{0}$, we set $\rho^{i_{0}}=\left(\rho_{s}^{i_{0}}, s \geq 0\right)$ where $\rho_{s}^{i_{0}}=\rho_{s}$ if $s<\alpha_{i_{0}}$ and $\rho_{s}^{i_{0}}=\rho_{s-\beta_{i_{0}}+\alpha_{i_{0}}}$ if $s>\beta_{i_{0}}$. Eventually, we set $\tilde{I}=\tilde{I}_{+} \cup \tilde{I}_{-} \cup\left\{i_{s}, i_{0}\right\}$. And $\left(\rho^{i}, i \in \tilde{I}\right)$ correspond to the fragments of the Lévy snake corresponding to the cutting of $\rho$ according to one point chosen with the measure $q_{\rho}$. We shall denote $\tilde{\nu}_{\rho}$ the distribution of $\left(\rho^{i}, i \in \tilde{I}\right)$ under $\mathbb{N}$.

In Section [9.3. we shall use $\sigma^{i}$, the length of fragment $\rho^{i}$. For $i \in \tilde{I}_{-} \cup \tilde{I}_{+}$, we have $\sigma^{i}=\beta_{i}-\alpha_{i}$. We also have $\sigma^{i_{s}}=\sigma_{-}^{i_{s}}+\sigma_{+}^{i_{s}}$ (resp. $\sigma^{i_{0}}=\sigma_{-}^{i_{0}}+\sigma_{+}^{i_{0}}$ ), where $\sigma_{-}^{i_{s}}=s-\alpha_{i_{s}}$ (resp. $\sigma_{-}^{i_{0}}=\alpha_{i_{0}}$ ) is the length of the fragment before $s$ and $\sigma_{+}^{i_{s}}=\beta_{i_{s}}-s$ (resp. $\sigma_{+}^{i_{0}}=\sigma-\beta_{i_{0}}$ ) is the length of the fragment after $s$. Notice that $\mathbb{N}$-a.e. $\sigma=\sum_{i \in \tilde{I}} \sigma^{i}$.

9.2. The dislocation process is a point process. Let $\mathcal{T}$ the set of time jumps of the Poisson process $\mathcal{Q}$. For $\theta \in \mathcal{T}$, consider $\mathcal{L}^{(\theta-)}=\left(\rho_{i}, i \in I^{(\theta-)}\right)$ and $\mathcal{L}^{(\theta)}=\left(\rho_{i}, i \in I^{(\theta)}\right)$ the families of Lévy snakes defined in Remark 8.2. The length, ranked in decreasing order, of those families of Lévy snakes correspond respectively to the fragmentation process just before time $\theta$ and at time $\theta$. Notice that for $\theta \in \mathcal{T}$ the families $\mathcal{L}^{(\theta-)}$ and $\mathcal{L}^{(\theta)}$ agree but for only one snake $\rho^{i_{\theta}} \in \mathcal{L}^{(\theta-)}$ which fragments in a family $\left(\rho^{i}, i \in \tilde{I}^{(\theta)}\right) \subset \mathcal{L}^{(\theta)}$. Thus we have that

$$
\mathcal{L}^{(\theta)}=\left(\mathcal{L}^{(\theta-)} \backslash\left\{\rho^{i_{\theta}}\right\}\right) \bigcup\left(\rho^{i}, i \in \tilde{I}^{(\theta)}\right) .
$$

From the representation of the previous Section, this fragmentation is given by cutting the Lévy snake according to the measure $q_{\rho}$ : that is the measure $\tilde{\nu}_{\rho}$ defined at the end of Section 9.1. From Lemma 8.6 and the construction of the Lévy Poisson Snake, we deduce that

$$
\sum_{\theta \in \mathcal{T}} \delta_{\theta, \mathcal{L}^{(\theta-)},\left(\rho^{i}, i \in \tilde{I}^{(\theta)}\right)}
$$

is a point process with intensity $d \theta \delta_{\mathcal{L}^{(\theta-)}} \sum_{\rho \in \mathcal{L}^{(\theta-)}} \tilde{\nu}_{\rho}$.

Notice the evolution of a tagged fragment of the Lévy snake has the same distribution as the evolution of the fragment of the Lévy snake which contains 0 , say $\rho^{0,(\theta)} \in \mathcal{L}^{(\theta)}$. (This is known as the re-rooting property of the CRT.) Then, we get that

$$
\sum_{\theta \in \mathcal{T}_{0}} \delta_{\theta,\left(\rho^{i}, i \in \tilde{I}^{(\theta)}\right)}=\sum_{\theta \in \mathcal{T}} \delta_{\theta,\left(\rho^{i}, i \in \tilde{I}^{(\theta)}\right)} \mathbf{1}_{\left\{0 \text { belongs to }\left(\rho^{i}, i \in \tilde{I}^{(\theta)}\right)\right\}}
$$

where $\mathcal{T}_{0}$ is the set of time fragmentation of the fragment which contains 0 , is a point process with intensity $d \theta \tilde{\nu}_{\rho^{0,(\theta-)}}$. 
Now, in the dislocation process of the fragmentation, $\delta(d \theta, d x)=\sum_{\theta^{\prime} \in \mathcal{T}_{0}} \delta_{\theta^{\prime}, x\left(\theta^{\prime}\right)}(d \theta, d x)$, the sequences $x\left(\theta^{\prime}\right)$ are the length ranked in decreasing order, $\left(\sigma^{i}, i \in \tilde{I}^{(\theta)}\right)$, of the Lévy snakes $\left(\rho^{i}, i \in \tilde{I}^{(\theta)}\right)$. Using a projection argument, one can check that the dislocation process is a point process with intensity $d \theta \nu_{\sigma^{0,(\theta-)}}$, where $\sigma^{0,(\theta-)}$ is the length of $\rho^{0,(\theta-)}$ and $\nu_{\sigma^{0,(\theta-)}}$ is the distribution of the decreasing lengths of Lévy snakes under $\tilde{\nu}_{\rho^{0,(\theta-)}}$, integrated w.r.t. to the law of $\rho^{0,(\theta-)}$ conditionally on $\sigma^{0,(\theta-)}$. More precisely we have $\pi_{*}(d r)$-a.e.

$$
\int_{\mathcal{S} \downarrow} F(x) \nu_{r}(d x)=\mathbb{N}_{r}\left[\int F\left(\left(\sigma^{i}, i \in \tilde{I}\right)\right) \tilde{\nu}_{\rho}\left(d\left(\rho^{i}, i \in \tilde{I}\right)\right)\right],
$$

for any non-negative measurable function $F$ defined on $\mathcal{S}^{\downarrow}$, where $\left(\sigma^{i}, i \in \tilde{I}\right)$ as to be understood as the family of length, of the fragments $\left(\rho^{i}, i \in \tilde{I}\right)$, ranked in decreasing size.

This prove that the dislocation process is a point process. And we will now explicit the family of dislocation measures $\left(\nu_{r}, r>0\right)$.

As computations are more tractable under $\mathbb{N}$ than under $\mathbb{N}_{r}$, we shall compute for $\lambda \geq 0$, and any non-negative measurable function, $F$, defined on $\mathcal{S}^{\downarrow}$

$$
\int_{\mathbb{R}_{+} \times \mathcal{S} \downarrow} \mathrm{e}^{-\lambda r} F(x) \pi_{*}(d r) \nu_{r}(d x) .
$$

From the definition of $\tilde{\nu}_{\rho}$, and using the notation at the end of Section 9.1 we get that this last quantity is equal to

$$
\mathbb{N}\left[\mathrm{e}^{-\lambda \sigma} \int q_{\rho}(d s, d a) F\left(\left(\sigma^{i}, i \in \tilde{I}\right)\right)\right]
$$

where $\left(\sigma^{i}, i \in \tilde{I}\right)$ as to be understood as the family of length ranked in decreasing size.

9.3. Computation of dislocation measures. In order to compute quantities like (48), we shall consider for $p>0, p^{\prime}>0$ and $h \in \mathcal{B}_{+}(\mathcal{M}((0,+\infty)))$

$$
A=\mathbb{N}\left[\mathrm{e}^{-\lambda \sigma} \int q_{\rho}(d s, d a)\left(\sigma^{i_{s}}+\sum_{i \in \tilde{I}_{-} \cup \tilde{I}_{+}} \sigma^{i}\right) \mathrm{e}^{-p \sigma^{i_{0}}-p^{\prime} \sigma^{i_{s}}} h\left(\sum_{i \in \tilde{I}_{-} \cup \tilde{I}_{+}} \delta_{\sigma^{i}}\right)\right] .
$$

As $q_{\rho}(d s, d a)=\frac{\kappa_{s}(d a)}{d_{s, a}-g_{s, a}}$ and since $d_{s, a}-g_{s, a}=\sigma^{i_{s}}+\sum_{i \in \tilde{I}_{-} \cup \tilde{I}_{+}} \sigma^{i}$, we get

$$
A=\mathbb{N}\left[\int_{0}^{\sigma} d s \int \kappa_{s}(d a) \mathrm{e}^{-(p+\lambda) \sigma^{i 0}-\left(p^{\prime}+\lambda\right) \sigma^{i s}} h\left(\sum_{i \in \tilde{I}_{-} \cup \tilde{I}_{+}} \delta_{\sigma^{i}}\right) \mathrm{e}^{-\lambda \sum_{i \in \tilde{I}_{-} \cup \tilde{I}_{+}} \sigma^{i}}\right] .
$$

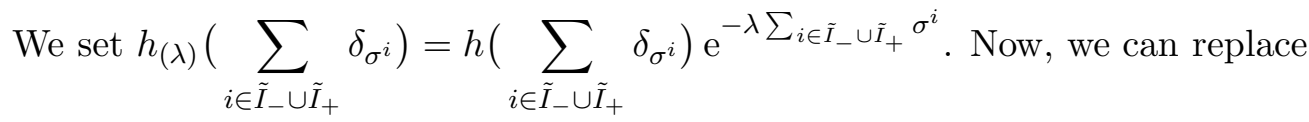

$$
\begin{aligned}
B & =\mathrm{e}^{-(p+\lambda) \sigma^{i_{0}}-\left(p^{\prime}+\lambda\right) \sigma^{i_{s}}} h_{(\lambda)}\left(\sum_{i \in \tilde{I}_{-} \cup \tilde{I}_{+}} \delta_{\sigma^{i}}\right) \\
& =\mathrm{e}^{-(p+\lambda) \sigma_{-}^{i_{0}}-\left(p^{\prime}+\lambda\right) \sigma_{-}^{i_{s}}-(p+\lambda) \sigma_{+}^{i_{0}}-\left(p^{\prime}+\lambda\right) \sigma_{+}^{i_{s}}} h_{(\lambda)}\left(\sum_{i \in \tilde{I}_{-}} \delta_{\sigma^{i}}+\sum_{i \in \tilde{I}_{+}} \delta_{\sigma^{i}}\right)
\end{aligned}
$$


by its optional projection $B^{\prime}$ :

$$
\begin{aligned}
& B^{\prime}=\mathrm{e}^{-(p+\lambda) \sigma_{-}^{i_{0}}-\left(p^{\prime}+\lambda\right) \sigma_{-}^{i_{s}}} \\
& \left.\quad \mathbb{E}_{\rho_{s}^{*}}^{*}\left[\mathrm{e}^{-(p+\lambda) \int_{0}^{\sigma} \mathbf{1}_{\left\{H_{0, u}<a\right\}} d u-\left(p^{\prime}+\lambda\right) \int_{0}^{\sigma} \mathbf{1}_{\left\{H_{0, u}>a\right\}} d u} h_{(\lambda)}\left(\mu^{\prime}+\sum_{j \in \tilde{I}_{+}} \delta_{\sigma^{j}}\right)\right]\right|_{\mu^{\prime}=\sum_{i \in \tilde{I}_{-} \delta_{\sigma^{i}}}} .
\end{aligned}
$$

Using notations introduced above Lemma 3.3. we have

$$
\begin{aligned}
& B^{\prime}=\mathrm{e}^{-(p+\lambda) \sigma_{-}^{i_{0}}-\left(p^{\prime}+\lambda\right) \sigma_{-}^{i_{s}}}
\end{aligned}
$$

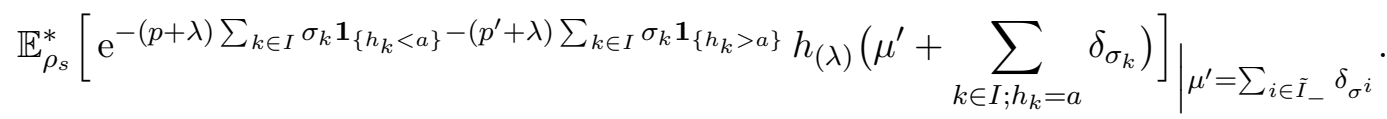

Then we deduce from Lemma 3.3, that

$$
\begin{aligned}
& B^{\prime}=\mathrm{e}^{-(p+\lambda) \sigma_{-}^{i_{0}}-\left(p^{\prime}+\lambda\right) \sigma_{-}^{i_{s}}} \\
& \left.\mathrm{e}^{-\rho_{s}([0, a)) \mathbb{N}\left[1-\mathrm{e}^{-(p+\lambda) \sigma}\right]-\rho_{s}((a,+\infty)) \mathbb{N}\left[1-\mathrm{e}^{-\left(p^{\prime}+\lambda\right) \sigma}\right]} \mathbb{E}\left[h_{(\lambda)}\left(\mu^{\prime}+\mathcal{P}\right)\right]\right|_{\mu^{\prime}=\sum_{i \in \tilde{I}_{-}} \delta_{\sigma^{i}}} \\
& =\left.\mathrm{e}^{-(p+\lambda) \sigma_{-}^{i_{0}}-\left(p^{\prime}+\lambda\right) \sigma_{-}^{i_{s}}} \mathrm{e}^{-\rho_{s}([0, a)) \psi^{-1}(p+\lambda)-\rho_{s}((a,+\infty)) \psi^{-1}\left(p^{\prime}+\lambda\right)} \mathbb{E}\left[h_{(\lambda)}\left(\mu^{\prime}+\mathcal{P}\right)\right]\right|_{\mu^{\prime}=\sum_{i \in \tilde{I}_{-}} \delta_{\sigma^{i}}},
\end{aligned}
$$

where $\mathcal{P}$ is under $\mathbb{P}$ a Poisson point measure with intensity $\rho_{s}(\{a\}) \mathbb{N}[d \sigma]=\rho_{s}(\{a\}) \pi_{*}(d r)$. By time reversibility (see Corollary 3.1.6 in [10]), we get

$$
\begin{aligned}
& A=\mathbb{N}\left[\int_{0}^{\sigma} d s \int \kappa_{s}(d a) \mathrm{e}^{-(p+\lambda) \sigma_{-}^{i_{0}}-\left(p^{\prime}+\lambda\right) \sigma_{-}^{i_{s}}}\right. \\
& \left.\left.\mathrm{e}^{-\rho_{s}([0, a)) \psi^{-1}(p+\lambda)-\rho_{s}((a,+\infty)) \psi^{-1}\left(p^{\prime}+\lambda\right)} \mathbb{E}\left[h_{(\lambda)}\left(\mu^{\prime}+\mathcal{P}\right)\right]\right|_{\mu^{\prime}=\sum_{i \in \tilde{I}_{-}} \delta_{\sigma^{i}}}\right] \\
& =\mathbb{N}\left[\int_{0}^{\sigma} d s \int \kappa_{s}(d a) \mathrm{e}^{-(p+\lambda) \sigma_{+}^{i_{0}}-\left(p^{\prime}+\lambda\right) \sigma_{+}^{i_{s}}}\right. \\
& \left.\left.\mathrm{e}^{-\eta_{s}([0, a)) \psi^{-1}(p+\lambda)-\eta_{s}((a,+\infty)) \psi^{-1}\left(p^{\prime}+\lambda\right)} \mathbb{E}\left[h_{(\lambda)}\left(\mu^{\prime}+\mathcal{P}^{\prime}\right)\right]\right|_{\mu^{\prime}=\sum_{i \in \tilde{I}_{+}} \delta_{\sigma^{i}}}\right],
\end{aligned}
$$

where $\mathcal{P}^{\prime}$ is under $\mathbb{P}$ a Poisson point measure with intensity $\eta_{s}(\{a\}) \pi_{*}(d \sigma)$. Using the same computation as above, we eventually get

$$
A=\mathbb{N}\left[\int_{0}^{\sigma} d s \int \kappa_{s}(d a) \mathrm{e}^{-\kappa_{s}([0, a)) \psi^{-1}(p+\lambda)-\kappa_{s}((a,+\infty)) \psi^{-1}(p+\lambda)} \mathbb{E}\left[h_{(\lambda)}\left(\mathcal{P}^{\prime \prime}\right)\right]\right]
$$

where $\mathcal{P}^{\prime \prime}$ is under $\mathbb{P}$ a Poisson point measure with intensity $\kappa_{s}(\{a\}) \pi_{*}(d \sigma)$. We write $g_{\lambda}\left(\kappa_{s}(\{a\})\right)$ for $\mathbb{E}\left[h_{(\lambda)}\left(\mathcal{P}^{\prime \prime}\right)\right]$. Thanks to the Poisson representation of Proposition [2.6] we 
get

$$
\begin{aligned}
A & =\mathbb{E}\left[\int_{0}^{\infty} d a \mathrm{e}^{-\alpha_{0} a} \sum_{x_{i} \leq a} \ell_{i} \mathrm{e}^{-\sum_{x_{j}<x_{i}} \ell_{j} \psi^{-1}(p+\lambda)-\sum_{a \geq x_{j}>x_{i}} \ell_{j} \psi^{-1}\left(p^{\prime}+\lambda\right)} g_{\lambda}\left(\ell_{i}\right)\right] \\
& =\int_{0}^{\infty} d a \mathrm{e}^{-\alpha_{0} a} \mathbb{E}\left[\sum_{x_{i} \leq a} \ell_{i} \mathrm{e}^{-x_{i} \int \ell \pi(d \ell)\left[1-\mathrm{e}^{-\ell \psi^{-1}(p+\lambda)}\right]-\left(a-x_{i}\right) \int \ell \pi(d \ell)\left[1-\mathrm{e}^{-\ell \psi^{-1}\left(p^{\prime}+\lambda\right)}\right]} g_{\lambda}\left(\ell_{i}\right)\right] \\
& =\int_{0}^{\infty} d a \mathbb{E}\left[\sum_{x_{i} \leq a} \ell_{i} \mathrm{e}^{-x_{i} \psi^{\prime}\left(\psi^{-1}(p+\lambda)\right)-\left(a-x_{i}\right) \psi^{\prime}\left(\psi^{-1}\left(p^{\prime}+\lambda\right)\right)} g_{\lambda}\left(\ell_{i}\right)\right] \\
& =\int_{0}^{\infty} d a \int_{(0, \infty)} \ell \pi(d \ell) \int d t \mathbf{1}_{[0, a]}(t) \ell \mathrm{e}^{-t \psi^{\prime}\left(\psi^{-1}(p+\lambda)\right)-(a-t) \psi^{\prime}\left(\psi^{-1}\left(p^{\prime}+\lambda\right)\right)} g_{\lambda}(\ell) \\
& =\frac{\int_{(0, \infty)} \ell^{2} g_{\lambda}(\ell) \pi(d \ell)}{\psi^{\prime}\left(\psi^{-1}(p+\lambda)\right) \psi^{\prime}\left(\psi^{-1}\left(p^{\prime}+\lambda\right)\right)},
\end{aligned}
$$

where we used (22) for the fourth equality.

On the other side, let $\left(\Delta S_{t}, t \geq 0\right)$ be the jumps of a subordinator $S=\left(S_{t}, t \geq 0\right)$ with Laplace exponent $\psi^{-1}$ and Lévy measure $\pi_{*}$. Standard computations yield for $r>0$,

$$
\begin{aligned}
G(r) & =\mathbb{E}\left[\mathrm{e}^{-\lambda S_{r}} \sum_{t \leq r, s \leq r, t \neq s} \Delta S_{t} \Delta S_{s} \mathrm{e}^{-p \Delta S_{t}-p^{\prime} \Delta S_{s}} h\left(\sum_{u \leq r, u \notin\{s, t\}} \delta_{\Delta S_{u}}\right)\right] \\
& =\mathbb{E}\left[\sum_{t \leq r, s \leq r, t \neq s} \Delta S_{t} \Delta S_{s} \mathrm{e}^{-(p+\lambda) \Delta S_{t}-\left(p^{\prime}+\lambda\right) \Delta S_{s}} h_{(\lambda)}\left(\sum_{u \leq r, u \notin\{s, t\}} \delta_{\Delta S_{u}}\right)\right] \\
& =r^{2}\left[\int \pi_{*}(d \ell) \ell \mathrm{e}^{-(p+\lambda) \ell}\right]\left[\int \pi_{*}(d \ell) \ell \mathrm{e}^{-\left(p^{\prime}+\lambda\right) \ell}\right] \mathbb{E}\left[h_{(\lambda)}\left(\sum_{u \leq r} \delta_{\Delta S_{u}}\right)\right] \\
& =r^{2} \psi^{-1^{\prime}}(p+\lambda) \psi^{-1^{\prime}}\left(p^{\prime}+\lambda\right) g_{\lambda}(r),
\end{aligned}
$$

as $\sum_{u \leq r} \delta_{\Delta S_{u}}$ is a Poisson measure with intensity $r \pi_{*}(d v)$. Notice that $\psi^{-1^{\prime}}=1 / \psi^{\prime} \circ \psi^{-1}$ to conclude that

$$
\int \pi(d r) G(r)=\frac{\int_{(0, \infty)} r^{2} g_{\lambda}(r) \pi(d r)}{\psi^{\prime}\left(\psi^{-1}(p+\lambda)\right) \psi^{\prime}\left(\psi^{-1}\left(p^{\prime}+\lambda\right)\right)}
$$

Therefore, we deduce that for any $p>0, p^{\prime}>0$ and $h \in \mathcal{B}_{+}(\mathcal{M}((0,+\infty)))$, we have

$$
\begin{aligned}
\mathbb{N}\left[\mathrm{e}^{-\lambda \sigma} \int q_{\rho}(d s, d a)\left(\sigma^{i_{s}}+\sum_{i \in \tilde{I}_{-} \cup \tilde{I}_{+}} \sigma^{i}\right) \mathrm{e}^{-p \sigma^{i_{0}}-p^{\prime} \sigma^{i_{s}}} h\left(\sum_{i \in \tilde{I}_{-} \cup \tilde{I}_{+}} \delta_{\sigma^{i}}\right)\right] \\
=\int \pi(d r) \mathbb{E}\left[\mathrm{e}^{-\lambda S_{r}} \sum_{t \leq r, s \leq r, t \neq s} \Delta S_{t} \Delta S_{s} \mathrm{e}^{-p \Delta S_{t}-p^{\prime} \Delta S_{s}} h\left(\sum_{u \leq r, u \notin\{s, t\}} \delta_{\Delta S_{u}}\right)\right] .
\end{aligned}
$$


Recall $\tilde{I}=\tilde{I}_{-} \cup \tilde{I}_{+} \cup\left\{i_{0}, i_{s}\right\}$. From monotone class Theorem, we deduce that for any $h \in$ $\mathcal{B}_{+}\left(\mathbb{R}_{+} \times \mathbb{R}_{+} \times \mathcal{M}((0,+\infty))\right)$,

$$
\begin{aligned}
\int \pi_{*}(d r) \mathrm{e}^{-\lambda r} \mathbb{N}_{r}\left[\int q(d s, d a) h\left(\sigma^{i_{0}}, \sigma^{i_{s}}, \sum_{i \in \tilde{I}-\cup \tilde{I}_{+}} \delta_{\sigma^{i}}\right)\right] \\
=\int \pi(d r) \mathbb{E}\left[S_{r} \mathrm{e}^{-\lambda S_{r}} \sum_{t \leq r, s \leq r, t \neq s} \frac{\Delta S_{t}}{S_{r}} \frac{\Delta S_{s}}{S_{r}-\Delta S_{t}} h\left(\Delta S_{t}, \Delta S_{s}, \sum_{u \leq r, u \notin\{s, t\}} \delta_{\Delta S_{u}}\right)\right] .
\end{aligned}
$$

For a measurable non-negative function $F$ defined on $\mathcal{S}^{\downarrow}$, we deduce that

$$
\int \pi_{*}(d r) \mathrm{e}^{-\lambda r} \mathbb{N}_{r}\left[\int q(d s, d a) F\left(\left(\sigma^{i}, i \in \tilde{I}\right)\right)\right]=\int \pi(d r) \mathbb{E}\left[S_{r} \mathrm{e}^{-\lambda S_{r}} F\left(\left(\Delta S_{u}, u \leq r\right)\right)\right],
$$

where $\left(\sigma^{i}, i \in \tilde{I}\right)$ and $\left(\Delta S_{u}, u \leq r\right)$ are to be understood as the family of length or jumps ranked in decreasing size. From the end of Section 9.2. we deduce that

$$
\int_{\mathbb{R}_{+} \times \mathcal{S} \downarrow} \mathrm{e}^{-\lambda r} F(x) \pi_{*}(d r) \nu_{r}(d x)=\int \pi(d v) \mathbb{E}\left[S_{v} \mathrm{e}^{-\lambda S_{v}} F\left(\left(\Delta S_{u}, u \leq v\right)\right)\right] .
$$

From definition (46) of $\mu$, we deduce that

$$
\int_{\mathbb{R}_{+} \times \mathcal{S} \downarrow} \mathrm{e}^{-\lambda r} F(x) \pi_{*}(d r) \nu_{r}(d x)=\int \mathrm{e}^{-\lambda r} F(x) r \mu(d r, d x) .
$$

This ends the proof of theorem 9.1 .

\section{REFERENCES}

[1] R. ABRAHAM and L. SERLET. Poisson snake and fragmentation. Elect. J. of Probab., 7, 2002.

[2] D. ALDOUS. The continuum random tree III. Ann. Probab., 21(1):248-289, 1993.

[3] D. ALDOUS and J. PITMAN. The standard additive coalescent. Ann. Probab., 26(4):1703-1726, 1998.

[4] J. BERTOIN. Lévy processes. Cambridge University Press, Cambridge, 1996.

[5] J. BERTOIN. A fragmentation process connected to Brownian motion. Probab. Th. Rel. Fields, 117:289301, 2000.

[6] J. BERTOIN. Self-similar fragmentations. Ann. Inst. Henri Poincaré, 38(3):319-340, 2000.

[7] J. BERTOIN. Random fragmentation and coagulation processes. To appear, 2006.

[8] J. BERTOIN, J.-F. LE GALL, and Y. LE JAN. Spatial branching processes and subordination. Canad. J. of Math., 49(1):24-54, 1997.

[9] R. BLUMENTHAL. Excursions of Markov processes. Birkhäuser, Boston, 1992.

[10] T. DUQUESNE and J.-F. LE GALL. Random trees, Lévy processes and spatial branching processes, volume 281. Astérisque, 2002.

[11] T. DUQUESNE and M. WINKEL. Growth of Lévy trees. To appear

[12] S. N. ETHIER and T. G. KURTZ. Markov processes. Wiley, 1986.

[13] J. JACOD. Calcul stochastique et problèmes de martingales, volume 714 of Lecture Notes in Mathematics. Springer, 1979.

[14] J.-F. LE GALL and Y. LE JAN. Branching processes in Lévy processes: Laplace functionals of snake and superprocesses. Ann. Probab., 26:1407-1432, 1998.

[15] J.-F. LE GALL and Y. LE JAN. Branching processes in Lévy processes: The exploration process. Ann. Probab., 26:213-252, 1998.

[16] G. MIERMONT. Ordered additive coalescent and fragmentations and stable subordinators. Elect. J. of Probab., 6, 2001.

[17] G. MIERMONT. Self-similar fragmentations derived from the stable tree i: splitting at heights. Probab. Th. Rel. Fields, 127(3):423-454, 2003.

[18] G. MIERMONT. Self-similar fragmentations derived from the stable tree ii: splitting at nodes. Probab. Th. Rel. Fields, (to appear), 2004. 
MAPMO, Université D'OrLÉANS, B.P. 6759, 45067 ORLÉANS CEDEX 2 FRANCE

E-mail address: romain.abraham@univ-orleans.fr

EnPC-CERMiCS, 6-8 av. Blaise Pascal, Champs-sur-Marne, 77455 Marne la Vallée, France.

E-mail address: delmas@cermics.enpc.fr 